\title{
A SPACE-TIME PROPERTY OF A CLASS OF MEASURE-VALUED BRANCHING DIFFUSIONS
}

\author{
EDWIN A. PERKINS
}

\begin{abstract}
If $d>\alpha$, it is shown that the $d$-dimensional branching diffusion of index $\alpha$, studied by Dawson and others, distributes its mass over a random support in a uniform manner with respect to the Hausdorff $\phi_{\alpha}$-measure, where $\phi_{\alpha}(x)=$ $x^{\alpha} \log \log 1 / x$. More surprisingly, it does so for all positive times simultaneously. Slightly less precise results are obtained in the critical case $d=\alpha$. In particular, the process is singular at all positive times a.s. for $d \geqslant \alpha$.
\end{abstract}

1. Introduction and statement of results. The critical multiplicative measure-valued diffusion process whose spatial diffusion is governed by a symmetric stable process can be used to model space-time properties of a population and has been studied by several authors (e.g. Watanabe [1968], Dawson [1975]). Much of the recent work on this and related processes has been on limit theorems of various sorts (e.g. Dawson [1977], Holley and Stroock [1978], Iscoe [1986], Cox and Griffeath [1985]). We study a sample path property of the process, namely the Hausdorff measure of its support as time varies. Questions of this sort have been answered at a fixed time but virtually nothing seems to be known about the nature of the random measure over finite time intervals.

$Y_{t}$ denotes a $d$-dimensional symmetric stable process of index $\alpha \in[0,2]$, starting at $x \in \mathbf{R}^{d}$ under the measure $P_{0}^{x}$, and scaled so that

$$
E_{0}^{0}\left(e^{i\left\langle\theta, Y_{t}\right\rangle}\right)=\exp \left(-t|\theta|^{\alpha}\right), \quad \theta \in \mathbf{R}^{d} .
$$

$A$ denotes the infinitesimal generator of $Y$ on $D(A) \subset C\left(\mathbf{R}^{d}\right)$, the space of bounded continuous real-valued functions on $\mathbf{R}^{d} . M\left(\mathbf{R}^{d}\right)$ is the set of measures on the Borel subsets of $\mathbf{R}^{d}, \mathscr{B}\left(\mathbf{R}^{d}\right)$, and $M_{F}\left(\mathbf{R}^{d}\right)$ is the set of finite measures in $M\left(\mathbf{R}^{d}\right)$ equipped with the topology of weak convergence of measures. We write $m(\phi)$ for the integral $\int \phi d m$. If $E$ is a metric space, $C([0, \infty), E)$ denotes the space of continuous functions from $[0, \infty)$ to $E$ with the compact-open topology, and $\mathscr{C}(E)$ is the class of Borel subsets.

Received by the editors October 6, 1986 .

1980 Mathematics Subject Classification (1985 Revision). Primary 60H15, 60J80, 60J60, 60G57; Secondary 28E05.

Key words and phrases. Measure-valued diffusion, Hausdorff measure, critical branching process, Loeb space.

Research partially supported by an NSERC of Canada operating grant. 
The measure-valued diffusions that we study may be characterized as solutions of a martingale problem (see Holley and Stroock [1978], Dawson and Kurtz [1982], Roelly-Coppoletta [1986]). The last reference contains a detailed proof of

THeOREM 1.1. If $m \in M_{F}\left(\mathbf{R}^{d}\right)$, there is a continuous $M_{F}$-valued, adapted process, $X_{t}$, defined on a filtered probability space $\left(\Omega, \mathscr{F}, \mathscr{F}_{t}, P\right)$ such that

(i) $P\left(X_{0}=m\right)=1$,

(ii) If $\phi \in D(A)$, then,

$$
X_{t}(\phi)=m(\phi)+\int_{0}^{t} X_{s}(A \phi) d s+Z_{t}(\phi),
$$

where $Z_{t}(\phi)$ is a continuous, $L^{2} \mathscr{F}_{t}$-martingale such that

$$
\langle Z(\phi)\rangle_{t}=\int_{0}^{t} X_{s}\left(\phi^{2}\right) d s .
$$

Moreover the law, $Q^{m}$, of $X$ on $C\left([0, \infty), M_{F}\right)$ is uniquely determined by (i) and (ii).

Setting $\phi \equiv 1$ in (ii) and passing to a larger probability space, if necessary, we obtain

THEOREM 1.2. If $X_{t}$ is as above, there is a one-dimensional Brownian motion, $B_{t}$, such that

$$
X_{t}(1)=m(1)+\int_{0}^{t} \sqrt{X_{s}(1)} d B_{s} .
$$

$X_{t}(1)$ is the continuous state branching process studied by Feller [1951].

Let $\left\{S_{t}: t \geqslant 0\right\}$ be the semigroup of $Y_{t}$ on $C\left(\mathbf{R}^{d}\right)$ and let $\phi \in C\left(\mathbf{R}^{d}\right)^{+}$(i.e., $\left.\phi \in C\left(\mathbf{R}^{d}\right), \phi \geqslant 0\right)$. If $u(t, x)$ is the unique solution of

$$
u(t, x)=S_{t} \phi(x)-\frac{1}{2} \int_{0}^{t} S_{t-s}\left(u(s, \cdot)^{2}\right) d s, \quad u(0, x)=\phi(x),
$$

then (see e.g. Roelly-Coppoletta [1986, Theorem 1.3]) the above process satisfies

$$
E\left(\exp \left(-X_{t}(\phi)\right)\right)=\exp \left(-\int u(t, x) d m(x)\right) \text {. }
$$

The right side is continuous in $m$ and standard arguments (Dawson and Kurtz [1982, Theorem 2.1]) show that Theorem 1.1 implies that $X$ is an $\mathscr{F}_{i}{ }^{-}$trong Markov process. We call $X$ the $d$-dimensional branching diffusion of index $\alpha$ starting at $m$. We will also assume (unless otherwise indicated) that $X_{t}$ is the coordinate process on $\left(C\left([0, \infty), M_{F}\right), \mathscr{C}\left(M_{F}\right), Q^{m}\right) . E_{Q}^{m}$ or $E^{m}$, if there is no ambiguity, denotes integration with respect to $Q^{m}$.

If $d<\alpha$, then for $t$ fixed $X_{t}$ has a density a.s. (Roelly-Coppoletta [1986]) and for $d=1, \alpha=2$, it has a density for all $t>0$ which is jointly continuous in $(t, x)$ a.s. (Reimers [1986]). We are interested in the case $d \geqslant \alpha$. Here, Dawson and Hochberg showed that $X_{t}$ is a.s. a singular measure for each $t>0$. In fact they showed more. Let $\operatorname{dim}(A)$ denote the Hausdorff dimension of $A \subset \mathbf{R}^{d}$. 
THEOREM 1.3. Assume $d \geqslant \alpha, t>0$ is fixed, and $m \in M_{F}\left(\mathbf{R}^{d}\right)$.

(a) (Dawson and Hochberg [1979]) $X_{t}$ is a singular measure and in fact is supported by a random Borel set, $\Lambda_{t}(\omega)$, such that $\operatorname{dim}\left(\Lambda_{t}(\omega)\right) \leqslant \alpha Q^{m}$-a.s.

(b) (U. Zähle [1984]) $X_{t}(A)=0$ for any $A \in \mathscr{B}\left(\mathbf{R}^{d}\right)$ satisfying $\operatorname{dim}(A)<\alpha$ $Q^{m}-a . s$.

The exceptional null set depends on $t$. A natural question, which I learned from Don Dawson, is therefore:

Does Theorem 1.3 hold for all $t>0$ a.s., or do there exist exceptional random times and spatial regions at which $X_{t}$ becomes more diffuse or more singular?

Another related problem is to find an exact Hausdorff measure function associated with $X_{t}$.

Notation. $\mathscr{H}=\left\{\phi:\left[0, \delta_{0}\right] \rightarrow[0, \infty): \delta_{0}>0, \phi(0)=0, \phi\right.$ strictly increasing and continuous\}.

If $\phi \in \mathscr{H}$ and $A \subset \mathbf{R}^{d}, \phi-m(A)$ denotes the Hausdorff $\phi$-measure of $A$, i.e.,

$$
\phi-m(A)=\lim _{\delta \rightarrow 0} \inf \left\{\sum_{i=1}^{\infty} \phi\left(d J_{i}\right): J_{i} \text { are balls of diameter } d J_{i} \leqslant \delta\right.
$$

$$
\text { such that } \left.A \subset \bigcup_{i=1}^{\infty} J_{i}\right\} \text {. }
$$

Is there a $\phi \in \mathscr{H}$ and a Borel support $\Lambda_{t}(\omega)$ of $X_{t}(\omega)$, such that $X_{t}(A)=\phi-m\left(A \cap \Lambda_{t}\right) \forall A \in \mathscr{B}\left(\mathbf{R}^{d}\right)$ ?

Notation. If $x>0$, let $\log ^{+} x=(\log x) \vee 0, \phi_{\alpha}(x)=x^{\alpha} \log ^{+} \log ^{+} 1 / x$,

$$
\begin{gathered}
\phi_{\alpha}^{(1)}(x)=x^{\alpha} \log ^{+} 1 / x, \quad \phi_{\alpha}^{(2)}(x)=x^{\alpha}\left(\log ^{+} 1 / x\right)\left(\log ^{+} \log ^{+} 1 / x\right), \\
\Phi_{\alpha}^{(3)}(x)=x^{\alpha}\left(\log ^{+} 1 / x\right)^{2} .
\end{gathered}
$$

Our main result answers (1.3) and comes close to answering (1.4) if $d>\alpha$. Recall $X_{t}$ is the $d$-dimensional branching diffusion of index $\alpha$ starting at $m \in M_{F}$ under $Q^{m}$ (as in Theorem 1.1).

THEOREM A. Let $d>\alpha$. There are constants $0<c(\alpha, d) \leqslant C(\alpha, d)<\infty$ such that for any $m \in M_{F}$ and $Q^{m}$-a.a. $\omega$ :

$\forall t>0$ there is a random Borel set $\Lambda_{t}(\omega)$ which supports $X_{t}$ and satisfies

$$
c(\alpha, d) \phi_{\alpha}-m\left(A \cap \Lambda_{t}\right) \leqslant X_{t}(A) \leqslant C(\alpha, d) \phi_{\alpha}-m\left(A \cap \Lambda_{t}\right) \quad \forall A \in \mathscr{B}\left(\mathbf{R}^{d}\right) .
$$

The theorem says that $X_{t}$ spreads its mass over its support in a very uniform manner and does so for all $t>0$ simultaneously. It is an immediate consequence of Theorems 4.5 and 6.3 below. An obvious conjecture, which we have been unable to verify, is

$$
\text { Theorem A holds with } c(\alpha, d)=C(\alpha, d) \text {. }
$$

In the critical case $d=\alpha(=1$ or 2$)$ our results are less precise but still answer (1.3). 
TheOREM B. Let $d=\alpha$. There are constants $0<c(\alpha, d) \leqslant C(\alpha, d)<\infty$ such that for any $m \in M_{F}$ and $Q^{m}$-a.a. $\omega$ :

$\forall t>0$ there is a random Borel support for $X_{t}, \Lambda_{t}$, which satisfies $c(\alpha, d) \phi_{\alpha}^{(1)}-m\left(A \cap \Lambda_{t}\right) \leqslant X_{t}(A) \leqslant C(\alpha, d) \phi_{\alpha}^{(3)}-m\left(A \cap \Lambda_{t}\right) \quad \forall A \in \mathscr{B}\left(\mathbf{R}^{d}\right)$.

If $t>0$ is fixed the result can be slightly improved.

TheOREM C. Let $d=\alpha$. There are constants $0<c(\alpha, d) \leqslant C(\alpha, d)<\infty$ such that for any $m \in M_{F}, t>0$ and $Q^{m}$-a.a. $\omega$ :

There is a random Borel support for $X_{t}, \Lambda_{t}$, which satisfies

$$
c(\alpha, d) \phi_{\alpha}^{(1)}-m\left(A \cap \Lambda_{t}\right) \leqslant X_{t}(A) \leqslant C(\alpha, d) \phi_{\alpha}^{(2)}-m\left(A \cap \Lambda_{t}\right) \quad \forall A \in \mathscr{B}\left(\mathbf{R}^{d}\right) .
$$

Theorem B follows from Theorems 4.6 and 6.4, while Theorem $\mathrm{C}$ is a consequence of $B$ and Theorem 4.7.

As an immediate corollary to $\mathrm{A}$ and $\mathrm{B}$, we have

Corollary D. If $d \geqslant \alpha$ and $m \in M_{F}$, then $X_{t}$ is purely singular (with respect to Lebesgue measure) for all $t>0 Q^{m}$-a.s.

The problem of finding an exact measure function for $X_{t}$ if $d=\alpha$ is left unresolved. A comparison of A and B shows that $X_{t}$ is "more singular" in this critical case. Our conjecture is

Theorem A remains valid if $d=\alpha$ and $\phi_{\alpha}$ is replaced by

$$
\phi_{\alpha}^{(4)}(x)=x^{\alpha}\left(\log ^{+} 1 / x\right)\left(\log ^{+} \log ^{+} \log ^{+} 1 / x\right) .
$$

It would also be of interest to see whether or not the above results remain valid if $\Lambda_{t}$ is the closed support of $X_{t}$.

All these theorems extend easily to the case when $X_{0}=m$ is an infinite measure in the class

$$
M_{p}\left(\mathbf{R}^{d}\right)=\left\{m \in M\left(\mathbf{R}^{d}\right): \int\left(1+|x|^{p}\right)^{-1} d m<\infty\right\},
$$

where if $\alpha \neq 2$ it is assumed that $p<d+\alpha$ (e.g. $m=$ Lebesgue measure). These extensions are described in $\$ 7$.

Our approach is based on the well-known construction of $X_{t}$ as the weak limit of a system of branching stable processes (Theorem 2.8 below). Those unfamiliar with this process may use Theorem 2.8 as its definition as it is more intuitive and no other properties of $X$ will be used in the proof of the main results. In $\$ 2$ a labelling system (borrowed from Walsh [1986]) is developed for the approximating systems of branching particles. This labelling system is an important tool as it allows us to prove sample path results by arguing directly with the sample paths.

To help describe the rest of the paper we first state a theorem of Rogers and Taylor [1961] that will play an important role. $B(x, r)$ denotes the closed ball in $\mathbf{R}^{d}$ centered at $x$ and of radius $r$. 
THEOREM 1.4. $\exists c(d)>0$ such that if $\phi \in \mathscr{H}, K>0$ and $\nu \in M_{F}\left(\mathbf{R}^{d}\right)$, then

(a) $\nu(A) \leqslant K \phi-m(A)$ whenever $A$ is a Borel subset of

$$
E_{1}(\nu, \phi, K)=\left\{x: \limsup _{a \downarrow 0} \nu(B(x, a)) \phi(a)^{-1} \leqslant K\right\} .
$$

(b) $\nu(A) \geqslant c(d) K \phi-m(A)$ whenever $A$ is a Borel subset of

$$
E_{2}(\nu, \phi, K)=\left\{x: \limsup _{a \downarrow 0} \nu(B(x, a)) \phi(a)^{-1} \geqslant K\right\} .
$$

(a) is a slight refinement of Lemma 3 of Rogers and Taylor [1961] because we have dropped a multiplicative constant $c^{\prime}(d)$ (note, however, $a$ is the radius of $B(x, a)$ and not its diameter). This refinement was pointed out by Martin Barlow. The proof is easy. (b) is a little more general than Lemma 2 of Rogers and Taylor, which only considers $A=E_{2}(\nu, \phi, K)$. The covering argument given there proves the result for closed subsets of $E_{2}$ and the inner regularity of $\phi-m$ (Rogers [1970, Theorems 47, 48 and the ensuing corollaries]) completes the proof.

Turning to Theorem A, we see that to apply the above result one needs probabilistic bounds on the $X_{t}$-measure of

$$
S_{1}=\left\{x: X_{t}(B(x, a)) \geqslant K \phi_{\alpha}(a)\right\}
$$

and

$$
S_{2}=\left\{x: X_{t}(B(x, a)) \leqslant K \phi_{\alpha}(a)\right\} .
$$

Estimates of this sort are given in $\S \S 3$ (for sets like $S_{1}$ ) and 5 (for sets like $S_{2}$ ). Instead of $X$, however, we work with the more explicit approximating systems of branching stable processes, $N^{(\mu)}(\mu \in \mathrm{N})$, defined below.

These estimates will be good enough to prove the results for fixed $t$ but interpolation arguments are needed to obtain the desired estimate for all $t \in\left[t_{0}, t_{0}+\Delta\right]$ simultaneously from the estimates at the endpoints. $\$ \$ 4$ and 6 contain the required interpolation arguments for the upper and lower bounds on $X_{t}$, respectively. In these sections we also complete the proofs of the main results by means of the limit theorem (Theorem 2.8) and the estimates on $N^{(\mu)}$. To carry out this last step we use nonstandard analysis and, in particular, the Loeb space construction (Loeb [1975]). It was tempting to fix an infinite $\mu$ and work with $N^{(\mu)}$ throughout as this would have made the proof shorter and more intuitive, but would have unfortunately cost us a considerable fraction of our audience. As it stands these final arguments may undoubtedly be standardized via weak convergence arguments but we felt the Loeb space offers such a superior approach (especially in conjunction with the labelling system) that it would be silly not to use it. Good introductions to the Loeb space construction, requiring no prior knowledge of nonstandard analysis, may be found in Loeb [1979] or Cutland [1983].

If $E$ is a metric space, $D(E)$ denotes the space of cadlag paths from $[0, \infty)$ to $E$ with the Skorokhod $J_{1}$-topology, $\mathscr{D}(E)$, the $\sigma$-field of Borel sets and $\left\{\mathscr{D}_{t}: t \geqslant 0\right\}=$ $\left\{\mathscr{D}_{t}(E): t>0\right\}$, the canonical right-continuous filtration. 
$c, c_{1}, c_{2}, \ldots$ will denote strictly positive constants whose value is unimportant and may change from line to line. $K^{(1)}, K^{(2)}, c^{(1)}, c^{(2)}, \ldots$ denote strictly positive constants that may play a role in subsequence arguments. Unless otherwise indicated, these constants may depend on $\alpha$ and $d$. Any other dependence will be explicitly stated. $(\alpha, d)$ will always satisfy $d \geqslant \alpha \in[0,2]$. If $A$ is a set, $I(A)$ denotes its indicator function and $A^{c}$ is its complement. $\mathbf{N}_{0}$ denotes the natural numbers including zero.

2. Branching stable processes. In this section we develop a rather elaborate labelling scheme for a system of branching stable processes. At first it may appear that this degree of precision is more trouble than its worth, but as the arguments get more involved the scheme will become a central component of our method. Most authors seem to have avoided such a system by arguing analytically rather than probabilistically. The latter approach seems more direct for our purposes.

Let $I=\bigcup_{n=0}^{\infty} \mathbf{N} \times\{0,1\}^{n}$. If $\beta=\left(\beta_{0}, \beta_{1}, \ldots, \beta_{j}\right) \in I$, let $|\beta|=j$ and $\beta \mid i=$ $\left(\beta_{0}, \ldots, \beta_{i}\right)$ for $i \leqslant j$. We call $|\beta|$ the length of the multi-index $\beta$. Let $\left\{Y^{\beta}: \beta \in I\right\}$ be a collection of independent $d$-dimensional symmetric stable processes of index $\alpha$, starting at zero and scaled so that (1.1) holds. Let $\left\{e^{\beta}: \beta \in I\right\}$ be a collection of independent random variables that take on the values 0 and 2 each with probability $1 / 2$. Assume these collections are mutually independent and defined on a common probability space, $\left(\Omega^{2}, \mathscr{A}^{2}, P^{2}\right)$. In fact we may, and shall, assume $\Omega^{2}=$ $\left(D\left([0, \infty), \mathbf{R}^{d}\right) \times\{0,2\}\right)^{I}, \mathscr{A}^{2}$ is the product $\sigma$-field and $P^{2}$ is the appropriate product probability. Let $\overline{\mathbf{R}^{d}}=\mathbf{R}^{d} \cup\{\Delta\}$ be the one-point compactification of $\mathbf{R}^{d}$, $\left(\Omega^{1}, \mathscr{A}^{1}\right)=\left(\left(\overline{\mathbf{R}}^{d}\right)^{\mathbf{N}}, \mathscr{B}\left(\overline{\mathbf{R}}^{\alpha}\right)^{\mathbf{N}}\right)$ (product space), and $(\Omega, \mathscr{A})=\left(\Omega^{1} \times \Omega^{2}, \mathscr{A}^{1} \times \mathscr{A}^{2}\right)$. If $\omega=\left(\omega^{1}, \omega^{2}\right)=\left(\left(x_{i}\right), \omega^{2}\right) \in \Omega$, we abuse the notation slightly and write $Y^{\beta}(\omega)$ and $e^{\beta}(\omega)$, for $Y^{\beta}\left(\omega^{2}\right)$ and $e^{\beta}\left(\omega^{2}\right)$, respectively. $\pi^{i}$ denotes the projection of $\Omega$ onto $\Omega^{i}$.

Fix $\mu \in \mathbf{N}$. In what follows dependence on $\mu$ is usually suppressed. Let $T=T^{(\mu)}$ $=\{j / \mu \mid j=0,1,2, \ldots\}$ and denote elements of $T$ by $\underline{s}, \underline{t}, \ldots$ We let $\lambda$ be the measure that assigns mass $1 / \mu$ to each point in $\{j / \mu: j \in Z\}$. If $\beta \in I$, $t \in[0,(1+|\beta|) / \mu)$ and $x_{\beta_{0}} \neq \Delta$, let

$$
\hat{N}_{t}^{\beta}\left(\left(x_{i}\right), \omega^{2}\right)=x_{\beta_{0}}+\sum_{i=0}^{|\beta|} \int I(i / \mu \leqslant s<t \wedge((i+1) / \mu)) d Y_{s}^{\beta \mid i} .
$$

Next use the $\left\{e^{\beta}\right\}$ to define killing times by

$$
\tau^{\beta}\left(\left(x_{i}\right), \omega^{2}\right)= \begin{cases}0 & \text { if } x_{\beta_{0}}=\Delta, \\ \min \left\{(i+1) / \mu: e^{\beta \mid i}=0\right\} & \text { if this set is nonempty and } x_{\beta_{0}} \neq \Delta, \\ (|\beta|+1) / \mu & \text { if this set is empty and } x_{\beta_{0}} \neq \Delta .\end{cases}
$$

Let

$$
N_{t}^{\beta}= \begin{cases}\hat{N}_{t}^{\beta} & \text { if } 0 \leqslant t<\tau^{\beta} \\ \Delta & \text { if } t \geqslant \tau^{\beta}\end{cases}
$$


$\left\{N^{\beta} \mid \beta \in I\right\}$ is a system of branching symmetric stable processes. Given $\omega=$ $\left(\left(x_{i}\right), \omega^{2}\right)$, particles start at each $x_{i} \neq \Delta$ and die or split into two particles with equal probability at the deterministic times in $T$. In between these times the particles move according to the law of a symmetric stable process of index $\alpha$. Each trajectory up to time $j / \mu$ is indexed by a $\beta \in I$ with $|\beta|=j$.

Notation. If $\beta \in I$ and $t \geqslant 0$, write $\beta \sim t$ iff $|\beta| / \mu \leqslant t<(|\beta|+1) / \mu$. $\delta_{x}$ denotes point mass at $x$ and $M_{F}^{\mu}=\left\{\mu^{-1} \sum_{i=1}^{K} \delta_{x_{i}}: K \in \mathbf{N}_{0}, x_{i} \in \mathbf{R}^{d}\right\} \subset M_{F}\left(\mathbf{R}^{d}\right)$.

Definition. $N=N^{(\mu)}:[0, \infty) \times \Omega \rightarrow M\left(\mathbf{R}^{d}\right)$ is given by

$$
N_{t}(\omega)=\frac{1}{\mu} \sum_{\beta \sim t} \delta_{N_{t}^{\beta}(\omega)} I\left(N_{t}^{\beta} \neq \Delta\right) .
$$

Using the notation introduced in $\S 1$, one has

$$
N_{t}(\phi)=\frac{1}{\mu} \sum_{\beta \sim t} \phi\left(N_{t}^{\beta}\right) \text { for } \phi: \mathbf{R}^{d} \rightarrow[0, \infty),
$$

where it is understood that $\phi(\Delta)=0$.

If $t \in[j / \mu,(j+1) / \mu)$ for some $j \in \mathbf{N}_{0}$, let

$$
\mathscr{A}_{t}=\sigma\left(\pi^{1}, Y^{\beta}, e^{\beta}:|\beta|<j\right) \vee\left(\bigcap_{u>t} \sigma\left(Y_{s}^{\beta}:|\beta|=j, s \leqslant u\right)\right), \quad \mathscr{A}_{\infty}=\mathscr{A} .
$$

To define a family of shift operators on $\Omega$, choose a sequence of bijections

$$
\psi_{j / \mu}: \mathbf{N} \rightarrow \mathbf{N} \times\{0,1\}^{j}, \quad j=0,1, \ldots,
$$

and define $\rho_{j / \mu}: I \rightarrow\{\beta \in I:|\beta| \geqslant j\}$ by

$$
\rho_{j / \mu}\left(\alpha_{0}, \ldots, \alpha_{n}\right)=\left(\psi_{j / \mu}\left(\alpha_{0}\right), \alpha_{1}, \ldots, \alpha_{n}\right) .
$$

For each $\underline{t} \in T$ there is a measurable, measure-preserving map $\theta_{t}^{2}: \Omega^{2} \rightarrow \Omega^{2}$ such that

$$
Y_{s}^{\beta} \circ \theta_{t}^{2}=Y_{s+t}^{\rho_{t}(\beta)}-Y_{t}^{\rho_{t}(\beta)}, \quad e^{\beta} \circ \theta_{t}^{2}=e^{\rho_{t}(\beta)} .
$$

Define the shift opertators $\theta_{t}: \Omega \rightarrow \Omega$ for $\underline{t} \in T$ by

$$
\theta_{t}\left(\omega^{1}, \omega^{2}\right)=\left(\left(N_{\underline{t}}^{\psi_{t}(i)}\left(\omega^{1}, \omega^{2}\right)\right)_{i}, \theta_{\underline{t}}^{2} \omega^{2}\right) .
$$

Finally we introduce a family of probabilities on $(\Omega, \mathscr{A})$. If $m=\mu^{-1} \sum_{i=1}^{K} \delta_{x_{i}}$ $\left(K \in \mathbf{N}_{0} \cup\{\infty\}\right.$ ), extend $\left\{x_{i}: i \leqslant K\right\}$ to $\mathbf{N}$ (if necessary) by setting $x_{i}=\Delta$ for $i>K$ and define $P^{m}=\delta_{\left(x_{i}\right)_{i \in N}} \times P^{2}$. The obvious drawback to this notation is that $P^{m}$ depends not only on $m$ but on the ordering of the $\left\{x_{i}\right\}$ (although this is not the case on $\sigma\left(N_{t}: t \geqslant 0\right)$ ). As a result it will sometimes be convenient to write $P_{(i)}^{\left(x_{i}\right)_{i} \text { for }}$ the above measure. If $m=N_{t^{\prime}}$ the notation $P^{N_{t}}$ will always refer to $\left.P^{\left(\psi_{t}(i)\right.}\right)_{i}$ and similarly if $\underline{t}$ is replaced with an $\left\{\mathscr{A}_{t}: \underline{t} \in T\right\}$-stopping time.

The following results are trivial from the above construction.

Lemma 2.1. (a) $N^{\beta} \in D\left([0, \infty), \overline{\mathbf{R}^{\alpha}}\right)$ and is $\left\{\mathscr{A}_{t}\right\}$-adapted.

(b) If $\beta, \gamma \in I$ satisfy $\beta|j=\gamma| j$ for some $j \leqslant|\beta|=|\gamma|$, then $N_{t}^{\beta}=N_{t}^{\gamma}$ for $t \leqslant$ $(j+1) / \mu$.

(c) If $\beta \in I, t \in[0,(|\beta|+1) / \mu),\left(x_{i}\right) \in \Omega^{1}$ satisfies $x_{\beta_{0}} \neq \Delta$, and $A \in \mathscr{D}_{t}$, then $P^{\left(x_{i}\right)}\left(N^{\beta} \in A \mid N_{t}^{\beta} \neq \Delta\right)=P_{0}^{x_{\beta}}(Y \in A)$. 
(d) $N_{t}(\phi)$ is $\mathscr{A}_{t}$-measurable for any Borel measurable $\phi: \mathbf{R}^{d} \rightarrow[0, \infty)$ and $t \geqslant 0$.

(e) If $m \in M_{F}^{\mu}$, then $N \in D\left([0, \infty), M_{F}^{\mu}\right) P^{m}$-a.s.

Proof. We only give the proof of (c). Under $P^{\left(x_{i}\right)}, \hat{N}_{t}^{\beta}$ is a symmetric stable process on $[0,(|\beta|+1) / \mu)$ starting at $x_{\beta_{0}}$ and is independent of $\tau^{\beta}$. Therefore

$$
P^{\left(x_{i}\right)}\left(N^{\beta} \in A \mid N_{t}^{\beta} \neq \Delta\right)=P^{\left(x_{i}\right)}\left(\hat{N}^{\beta} \in A \mid \tau^{\beta}>t\right)=P_{0}^{x_{\beta_{0}}}(Y \in A) .
$$

Proposition 2.2. If $m \in M_{F}^{\mu}$ and $\phi: \mathbf{R}^{d} \rightarrow[0, \infty)$ is Borel measurable, then

$$
E^{m}\left(N_{t}(\phi)\right)=\int E_{0}^{x}\left(\phi\left(Y_{t}\right)\right) d m(x) \equiv E_{0}^{m}\left(\phi\left(Y_{t}\right)\right), \quad t \geqslant 0 .
$$

Proof. Let $m=\mu^{-1} \sum_{i=1}^{K} \delta_{x_{i}}$.

$$
\begin{aligned}
E^{m}\left(N_{t}(\phi)\right) & =\mu^{-1} \sum_{i=1}^{K} \sum_{\beta \sim t, \beta_{0}=i} E^{m}\left(\phi\left(N_{t}^{\beta}\right) \mid N_{t}^{\beta} \neq \Delta\right) P\left(N_{t}^{\beta} \neq \Delta\right) \\
& =\mu^{-1} \sum_{i=1}^{K} E_{0}^{x_{i}}\left(\phi\left(Y_{t}\right)\right) E\left(\sum_{\beta \sim t, \beta_{0}=i} I\left(N_{t}^{\beta} \neq \Delta\right)\right) \quad(\text { Lemma 2.1(c)) } \\
& =\mu^{-1} \sum_{i=1}^{K} E_{0}^{x_{i}}\left(\phi\left(Y_{t}\right)\right) \quad \text { (critical branching). }
\end{aligned}
$$

The Markov property for $N_{t}$ is intuitively obvious but we must check that our shift operators are well behaved.

Proposition 2.3. (a) If $\underline{t} \in T, s \geqslant 0$ and $\beta \in I$,

$$
\begin{aligned}
N_{s}^{\beta} \circ \theta_{t} & =N_{s+t}^{\rho}(\beta), \\
N_{s} \circ \theta_{t} & =N_{s+\underline{t}} .
\end{aligned}
$$

(b) Let $m \in M_{F}^{\mu}$ and $U: \Omega \rightarrow T \cup\{\infty\}$ be an $\left\{\mathscr{A}_{t}\right\}$-stopping time (i.e. $\{U=\underline{t}\}$ $\left.\in \mathscr{A}_{\underline{t}} \forall \underline{t} \in T \cup\{\infty\}\right)$. Then for any $A \in \mathscr{A}$,

$$
E^{m}\left(I_{A} \circ \theta_{U} \mid \mathscr{A}_{U}\right)=P^{N_{U}}(A) \quad P^{m} \text {-a.s. on }\{U<\infty\} \text {. }
$$

Proof. (a) Let $\underline{t} \in T, \beta \in I$ and $s \geqslant 0$. If $s<(|\beta|+1) / \mu$ and $\tau^{\rho_{t}(\beta)}>\underline{t}$, then

$$
\begin{aligned}
\hat{N}_{s}^{\beta} \circ \theta_{t}(\omega) & =N_{\underline{t}}^{\psi_{t}\left(\beta_{0}\right)}+\sum_{i=0}^{|\beta|} \int I\left(\frac{i}{\mu} \leqslant u<s \wedge \frac{i+1}{\mu}\right) d Y_{u}^{\beta \mid i} \circ \theta_{\underline{t}}^{2}\left(\omega^{2}\right) \\
& =N_{\underline{t}}^{\rho_{t}(\beta) \mid \mu t}+\sum_{i=0}^{|\beta|} \int I\left(\frac{i}{\mu} \leqslant u<s \wedge \frac{i+1}{\mu}\right) d Y_{u+t}^{\rho_{t}(\beta \mid i)} \\
& =\hat{N}_{\underline{t}}^{\rho_{t}(\beta) \mid \mu t}+\sum_{i=\mu t}^{\left|\rho_{t}(\beta)\right|} \int I\left(\frac{i}{\mu} \leqslant u<(s+\underline{t}) \wedge \frac{i+1}{\mu}\right) d Y_{u}^{\rho_{t}(\beta) \mid i} \\
(2.3) \quad\left(\rho_{\underline{t}}(\beta \mid i)=\rho_{\underline{t}}(\beta) \mid(i+\mu \underline{t})\right), & \hat{N}_{s}^{\beta} \circ \theta_{t}(\omega)=\hat{N}_{s+\underline{t}}^{\rho_{t}(\beta)} .
\end{aligned}
$$


If $\tau^{\rho_{t}(\beta)}(\omega) \in\left(\underline{t}, \underline{t}+\left(\left|\rho_{t}(\beta)\right|+1\right) / \mu\right)$, then

$$
\begin{aligned}
\tau^{\beta} \circ \theta_{t}(\omega) & =\min \left\{(i+1) / \mu: e^{\beta \mid i} \circ \theta_{t}(\omega)=0\right\} \\
& =\min \left\{(i+1) / \mu: e^{\rho_{t}(\beta) \mid i+\mu t}(\omega)=0\right\}=\tau^{\rho_{t}(\beta)}(\omega)-\underline{t} .
\end{aligned}
$$

Similar reasoning gives the same result if $\tau^{\rho_{t}(\beta)}(\omega)=\left(\left|\rho_{t}(\beta)\right|+1\right) / \mu$, and if $\tau^{\rho_{t}(\beta)}(\omega) \leqslant \underline{t}$, then $\tau^{\beta} \circ \theta_{t}(\omega)=0$ because $N_{t}^{\psi_{t}\left(\beta_{0}\right)}=N_{t}^{\rho_{t}(\beta)}=\Delta$. We have shown that $\tau^{\beta} \circ \theta_{t}=\left(\tau^{\rho_{t}(\beta)}-\underline{t}\right)^{+}$. This, together with (2.3), gives (2.1). (2.2) follows from

$$
N_{s} \circ \theta_{t}=\frac{1}{\mu} \sum_{\beta \sim s} \delta_{N_{s}^{\beta} \circ \theta_{t}}=\frac{1}{\mu} \sum_{\beta \sim s} \delta_{\substack{N_{s+t} \times(\beta) \\ s+\underline{t}}}=N_{s}
$$

The last equality holds because $\rho_{t}$ is a bijection between $\{\beta: \beta \sim s\}$ and $\{\beta: \beta \sim s+\underline{t}\}$.

(b) We may assume that $U=\underline{t}$ is constant because the time set is discrete. Let $m=\mu^{-1} \sum_{i=1}^{K} \delta_{x_{i}} \in M_{F}^{\mu}$. It suffices to prove the result for

$$
A=\left\{\omega: \omega^{1} \in A_{0}, Y^{\beta_{i}} \in B_{i}, e^{\beta_{i}} \in C_{i}, i=1, \ldots, n\right\},
$$

where $\beta_{i} \in I, A_{0} \in \mathscr{A}^{1}, B_{i} \in \mathscr{D}\left(\mathbf{R}^{d}\right)$, and $C_{i} \in \mathscr{B}(\mathbf{R})$. In this case one has $P^{m}\left(I_{A} \circ \theta_{t} \mid \mathscr{A}_{\underline{t}}\right)=P^{m}\left(\left(N_{\underline{t}}^{\psi_{t}(i)}\right)_{i} \in A_{0}, Y_{\underline{t}+}^{\rho\left(\beta_{i}\right)}-Y_{\underline{t}}^{\rho_{t}\left(\beta_{i}\right)} \in B_{i}, e^{\rho_{t}\left(\beta_{i}\right)} \in C_{i}, i \leqslant n \mid \mathscr{A}_{t}\right)$. $\left|\rho_{t}\left(\beta_{i}\right)\right| \geqslant \mu t$ implies that $\left(Y^{\rho_{t}\left(\beta_{i}\right)}\right)_{i}$ and $\left(e^{\rho_{t}\left(\beta_{i}\right)}\right)_{i}$ are mutually independent and independent of $\mathscr{A}_{t}$, and therefore the above equals

$$
I\left(\left(N_{\underline{t}}^{\psi_{t}(i)}\right)_{i} \in A_{0}\right) P^{2}\left(Y^{\beta_{i}} \in B_{i}, e^{\beta_{i}} \in C_{i}, i \leqslant n\right)=P^{N_{t}}(A) .
$$

Notation. If $S \subset I$, let

$$
\begin{aligned}
\mathscr{F}(S) & =\sigma\left(\pi^{1}, Y^{\beta \mid k}, e^{\beta \mid k}: \beta \in S, 0 \leqslant k \leqslant|\beta|\right), \\
\mathscr{G}(S) & =\sigma\left(Y^{\beta}, e^{\beta}: \beta \in S\right) .
\end{aligned}
$$

If $\beta \in I$ and all the indices in $S$ have length $|\beta|$, define

$$
\sigma(S ; \beta)= \begin{cases}|\beta|-\inf \{j: \beta|j \neq \gamma| j \text { for all } \gamma \in S\} & \text { if } \beta \notin S, \\ -1 & \text { if } \beta \in S .\end{cases}
$$

$\sigma(S ; \beta)$ is the number of "generations" back that $\beta$ first split off from the family tree generated by $S$. Write $\sigma(\gamma ; \beta)$ for $\sigma(\{\gamma\} ; \beta)$.

If $\phi: \mathbf{R}^{d} \rightarrow[0, \infty)$ is Borel measurable and $\underline{t} \in T$, let

$$
G(\phi, \underline{t})=\int_{[0, t]} \sup _{y} E_{0}^{y}\left(\phi\left(Y\left(\left(\underline{s}-\mu^{-1}\right)^{+}\right)\right)\right) d \lambda(\underline{s}) .
$$

LEMMA 2.4. Let $S \subset I$ be a set of $n$ multi-indices each of length $\mu \underline{t}$. Assume $m \in M_{F}^{\mu}$ and $\phi: \mathbf{R}^{d} \rightarrow[0, \infty)$ is Borel measurable.

(a) If $\beta \in I, \beta \sim t$, and $\sigma(S ; \beta) \leqslant \mu t$, then for some $\gamma \in S, N_{t}^{\beta}=N_{t}^{\gamma}$ for $t<\underline{t}-\sigma(S ; \beta) / \mu$. Therefore $N_{t}^{\beta}$ is $\mathscr{F}(S)$-measurable for $t \leqslant \underline{t}-\sigma(S ; \beta) / \mu$ and $\sigma(S ; \beta) \leqslant \mu \underline{t}$.

(b) If $i \in\{-1,0, \ldots, \mu \underline{t}-1\}$, then

$$
E^{m}\left(\sum_{\beta: \sigma(S ; \beta)=i} \phi\left(N_{t}^{\beta}\right) \mid \mathscr{F}(S)\right) \leqslant n \sup _{\beta \in S}\left(E_{0}^{N_{t-i^{+} / \mu}^{\beta}}\left(\phi\left(Y_{i^{+} / \mu}\right)\right) I\left(N_{t-i^{+} / \mu}^{\beta} \neq \Delta\right)\right) .
$$

and equality holds if $n=1$. 
(c) If $0 \leqslant b<\underline{t}$, then

$$
E^{m}\left(\mu^{-1} \sum_{\beta: \sigma(S ; \beta) \leqslant \mu b} \phi\left(N_{\underline{t}}^{\beta}\right) \mid \mathscr{F}(S)\right) \leqslant n G(\phi,(1+[b \mu]) / \mu) .
$$

Proof. (a) If $\sigma(S ; \beta) / \mu=t$, the latter result is obvious because $N_{0}^{\beta}$ is $\sigma\left(\pi^{1}\right)$-measurable. If $\sigma(S ; \beta)<|\beta|$, the definition of $\sigma$ shows there is a $\gamma$ in $S$ such that $\gamma|(|\beta|-\sigma(S ; \beta)-1)=\beta|(|\beta|-\sigma(S ; \beta)-1)$, and therefore $N_{t}^{\beta}=N_{t}^{\gamma}$ for $t \leqslant \underline{t}-\sigma(S ; \beta) / \mu($ Lemma $2.1(\mathrm{~b}))$.

(b) Fix $\beta \in I$ such that $\beta \sim \underline{t}$ and $\sigma(S ; \beta)=i<|\beta|$. Then

$$
\{\beta|k:| \beta|-i \leqslant k \leqslant| \beta \mid\} \cap\{\gamma|k: \gamma \in S, k \leqslant| \beta \mid\}=\varnothing
$$

and therefore $\sigma\left\{Y^{\beta \mid k}, e^{\beta \mid k}:|\beta|-i \leqslant k \leqslant|\beta|\right\}$ is independent of $\mathscr{F}(S)$. By the above, $N_{\underline{t}-i^{+} / \mu}^{\beta}$ is $\mathscr{F}(S)$-measurable and hence

$$
\begin{aligned}
& E^{m}\left(\phi\left(N_{t}^{\beta}\right) \mid \mathscr{F}(S)\right)=I\left(N_{\underline{t}-i^{+} / \mu}^{\beta} \neq \Delta\right) \\
& \times E^{m}\left(I\left(e^{\beta \mid k}=2, k=|\beta|-i^{+}, \ldots,|\beta|-1\right)\right. \\
& \left.\quad \times \phi\left(N_{t-i^{+} / \mu}^{\beta}+\sum_{k=|\beta|-i^{+}}^{|\beta|-1} Y^{\beta \mid k}((k+1) / \mu)-Y^{\beta \mid k}(k / \mu)\right) \mid \mathscr{F}(S)\right) \\
& =I\left(N_{t-i^{+} / \mu}^{\beta} \neq \Delta\right) 2^{-i^{+}} E_{0}^{N_{t-i^{+} / \mu}^{\beta}}\left(\phi\left(Y\left(i^{+} / \mu\right)\right)\right) .
\end{aligned}
$$

Use the inclusion $\{\beta: \sigma(S ; \beta)=i\} \subset \bigcup_{\gamma \in S}\{\beta: \sigma(\gamma ; \beta)=i\}$ and the fact that each of these $n$ sets contains $2^{i^{+}}$elements (this requires $i<|\beta|$ ) to see that

$$
\begin{aligned}
& E^{m}\left(\sum_{\beta: \sigma(S ; \beta)=i} \phi\left(N_{t}^{\beta}\right) \mid \mathscr{F}(S)\right) \\
& \quad \leqslant n \sup \left\{I\left(N_{t-i^{+} / \mu}^{\beta} \neq \Delta\right) E_{0}^{N_{t-i^{+} / \mu}^{\beta}}\left(\phi\left(Y\left(i^{+} / \mu\right)\right)\right): \beta \sim \underline{t}, \sigma(S ; \beta)=i\right\} \\
& \quad \leqslant n \sup _{\beta \in S} E_{0}^{N_{t-i^{+} / \mu}^{\beta}}\left(\phi\left(Y\left(i^{+} / \mu\right)\right)\right) I\left(N_{\underline{t}-i^{+} / \mu}^{\beta} \neq \Delta\right) \quad(\text { by }(\mathrm{a})) .
\end{aligned}
$$

It is clear that one gets equality if $n=1$.

(c) follows easily from (b) by summing over $i$.

An argument similar to the proof of (b) above gives the next

LEMMA 2.5. Let $\underline{t} \in T$ and let $S$ be a set of multi-indices of length $\mu \underline{t}$. Suppose $\gamma_{1}$ and $\gamma_{2}$ satisfy $\left|\gamma_{1}\right|=\left|\gamma_{2}\right|=\mu \underline{t}, \sigma\left(S ; \gamma_{1}\right)=j, \sigma\left(S ; \gamma_{2}\right)=k$, and $\sigma\left(\gamma_{1} ; \gamma_{2}\right) \geqslant j \wedge k$. If $a>0$, then

(a)

$$
\begin{aligned}
P\left(\left|N_{\underline{t}}^{\gamma_{1}}-N_{\underline{t}}^{\gamma_{2}}\right| \leqslant a \mid \mathscr{F}(S) \vee \sigma\left(e^{\beta}: \beta \in I\right)\right) \\
\quad=P_{0}^{N_{l-j^{+} / \mu}^{\gamma_{1}}-N_{t}^{\gamma_{2}} k^{+} / \mu}\left(\left|Y\left(\left(j^{+}+k^{+}\right) / \mu\right)\right| \leqslant a\right) I\left(N_{\underline{t}}^{\gamma_{1}} \neq \Delta, N_{\underline{t}}^{\gamma_{2}} \neq \Delta\right),
\end{aligned}
$$


(b)

$$
\begin{aligned}
P\left(\left|N_{\underline{t}}^{\gamma_{1}}-N_{t}^{\gamma_{2}}\right| \leqslant a \mid \mathscr{F}(S)\right)= & 2^{-j^{+}-k^{+}} P_{0}^{N_{t}^{\gamma_{j}+/ \mu}}\left(\left|Y\left(\left(j^{+}+k^{+}\right) / \mu\right)\right| \leqslant a\right) \\
& \times I\left(N_{t}^{\gamma_{1} j^{+} / \mu} \neq \Delta, N_{t}^{\gamma_{2}} k^{+} / \mu\right.
\end{aligned}
$$

REMARK. (b) clearly follows from (a) by conditioning with respect to $\mathscr{F}(S)$. It is understood in the above that the inequality $\left|N_{t}^{\gamma_{1}}-N_{t}^{\gamma_{2}}\right| \leqslant a$ necessarily implies $N_{t}^{\gamma_{1}} \neq \Delta, N_{t}^{\gamma_{2}} \neq \Delta$.

To get a sharper estimate on $N_{t}(\phi)$ we will use the method of moments to get a bound on $E^{m}\left(e^{\theta N_{t}(\phi)}\right)$. This is reminiscent of the interesting technique used by Cox and Griffeath [1985] to obtain similar estimates on $\int_{0}^{t} N_{s}(\phi) d s$. They were interested in limit theorems as $t \rightarrow \infty$ and obtained a precise recursion relation between the moments of $\int_{0}^{t} N_{s}(\phi) d s$. We give a short probabilistic argument which takes advantage of our labelling system. The result represents one of the key estimates in the paper.

PRoposition 2.6. Let $\phi: \mathbf{R}^{d} \rightarrow[0, \infty]$ be Borel measurable, $x \in \mathbf{R}^{d}, m \in M_{F}^{\mu}$, $p \in \mathbf{N}$ and $\underline{t} \in T$.

(a)

$$
\begin{aligned}
& E^{\mu^{-1} \delta_{x}}\left(\mu N_{t}(\phi)^{p}\right) \leqslant(p-1) ! G(\phi, \underline{t})^{p-1} E_{0}^{x}\left(\phi\left(Y_{\underline{t}}\right)\right), \\
& E^{\mu^{-1} \delta_{x}}\left(\mu N_{t}(\phi)^{2}\right)=E_{0}^{x}\left(\phi\left(Y_{t}\right) \int_{\left[-\mu^{-1}, \underline{t}\right)} S_{\underline{u^{+}}} \phi\left(Y_{t^{-}-\underline{u}^{+}}\right) d \lambda(\underline{u})\right) .
\end{aligned}
$$

(b) If $\theta \in\left[0, G(\phi, \underline{t})^{-1}\right)$, then

$$
E^{\mu^{-1} \delta_{x}}\left(e^{\theta N_{t}(\phi)}\right) \leqslant 1+\theta \mu^{-1}(1-\theta G(\phi, \underline{t}))^{-1} E_{0}^{x}\left(\phi\left(Y_{t}\right)\right) .
$$

(c) If $\theta \in\left[0, G(\phi, \underline{t})^{-1}\right)$, then

$$
E^{m}\left(e^{\theta N_{t}(\phi)}\right) \leqslant \exp \left\{\theta(1-\theta G(\phi, \underline{t}))^{-1} E_{0}^{m}\left(\phi\left(Y_{t}\right)\right)\right\} .
$$

Proof. (a) Fix $x \in \mathbf{R}^{d}$, let $x_{1}=x$ and $x_{i}=\Delta$ for $i>1$, and write $P$ and $E$ for $P^{\left(x_{i}\right)}=P^{\mu^{-1} \delta_{x}}$ and $E^{\left(x_{i}\right)}=E^{\mu^{-1} \delta_{x}}$. Then for $p \in \mathbf{N} \geqslant 2$,

$$
\begin{aligned}
& E\left(\mu N_{t}(\phi)^{p}\right)=\mu^{-(p-1)} \sum_{\substack{\beta^{2} \sim t \\
\beta_{0}^{1}-1}} \cdots \sum_{\substack{\beta^{p}-t \\
\beta b-1}} E\left(\prod_{i=1}^{p} \phi\left(N_{t}^{\beta^{i}}\right)\right) \\
& =\mu^{-(p-2)} \sum_{\substack{\beta^{1}-1 \\
\beta_{0}^{1}-1}} \cdots \sum_{\substack{\beta^{p-1}-1 \\
\beta b^{-1}-1}} E\left(\prod_{i=1}^{p-1} \phi\left(N_{l}^{\beta^{i}}\right)\right. \\
& \left.\times E\left(\mu^{-1} \sum_{\substack{\beta^{p}-t, \sigma\left(\left\{\beta^{1} \cdots \beta^{p-1}\right\} ; \beta^{p}\right) \leqslant \mu t-1}} \phi\left(N_{l}^{\beta^{p}}\right) \mid \mathscr{F}\left(\beta^{1} \cdots \beta^{p-1}\right)\right)\right) \\
& \leqslant E\left(\mu N_{t}(\phi)^{p-1}\right)(p-1) G(\phi, t) \quad(L e m m a 2.4(\mathrm{c})) \text {. }
\end{aligned}
$$


The obvious induction and Proposition 2.2 complete the proof of (i). For (ii) note that

$$
\begin{aligned}
& E\left(\mu N_{t}(\phi)^{2}\right)=\mu^{-1} \sum_{\beta \sim t, \beta_{0}=1} E\left(\phi\left(N_{t}^{\beta}\right) \sum_{i=-1}^{\mu t-1} \sum_{\gamma: \sigma(\beta ; \gamma)=i} E\left(\phi\left(N_{t}^{\gamma}\right) \mid \mathscr{F}(\beta)\right)\right) \\
& =\mu^{-1} \sum_{\beta \sim t, \beta_{0}=1} E\left(\phi\left(N_{!}^{\beta}\right) \sum_{i=-1}^{\mu t-1} S_{i^{+} / \mu} \phi\left(N_{t-i^{+} / \mu}^{\beta}\right)\right) \\
& \text { (by Lemma 2.4(b) with } n=1 \text { ) } \\
& =\sum_{\beta \sim \underline{t}, \beta_{0}=1} E\left(\phi\left(N_{\underline{t}}^{\beta}\right) \int_{\left[-\mu^{-1}, t\right)} S_{\underline{u}^{+}} \phi\left(N_{\underline{t}-\underline{u}^{+}}^{\beta}\right) d \lambda(\underline{u}) \mid N_{\underline{t}}^{\beta} \neq \Delta\right) P\left(N_{\underline{t}}^{\beta} \neq \Delta\right) \\
& =E_{0}^{x}\left(\phi\left(Y_{\underline{t}}\right) \int_{\left[-\mu^{-1}, \underline{t}\right)} S_{\underline{u}^{+}} \phi\left(Y_{\underline{t}-\underline{u}^{+}}\right) d \lambda(\underline{u})\right)
\end{aligned}
$$

(Lemma 2.1(c), Proposition 2.2).

(b) If $0 \leqslant \theta<G(\phi, \bar{t})^{-1}$, (a) implies

$$
\begin{aligned}
E\left(e^{\theta N_{t}(\phi)}\right) & \leqslant 1+E_{0}^{x}\left(\phi\left(Y_{\underline{t}}\right)\right) \mu^{-1}\left(\sum_{p=1}^{\infty} \theta^{p} G(\phi, \underline{t})^{p-1} p^{-1}\right) \\
& \leqslant 1+\theta \mu^{-1} E_{0}^{x}\left(\phi\left(Y_{\underline{t}}\right)\right)(1-\theta G(\phi, \underline{t}))^{-1} .
\end{aligned}
$$

(c) Let $\theta \in\left[0, G(\phi, \underline{t})^{-1}\right)$ and $m=\mu^{-1} \sum_{i=1}^{K} \delta_{x_{i}}$. If

$$
N_{\underline{t}}^{(i)}=\mu^{-1} \sum_{\beta \sim t, \beta_{0}=i} \delta_{N_{t}^{\beta}}
$$

then (under $\left.P^{m}=P^{\left(x_{i}\right)}\right)\left\{N^{(i)}: i \leqslant K\right\}$ are independent and

$$
P^{m}\left(N^{(i)} \in \cdot\right)=P^{\mu^{-1} \delta_{x_{i}}}(N \in \cdot) .
$$

Therefore

$$
\begin{aligned}
E^{m}\left(e^{\theta N_{t}(\phi)}\right) & =\prod_{i=1}^{K} E^{\mu^{-1} \delta_{x_{i}}}\left(e^{\theta N_{t}(\phi)}\right) \\
& \leqslant \exp \left\{\theta \sum_{i=1}^{K} \mu^{-1} E_{0}^{x_{i}}\left(\phi\left(Y_{\underline{t}}\right)\right)(1-\theta G(\phi, \underline{t}))^{-1}\right\} \\
& =\exp \left\{\theta(1-\theta G(\phi, \underline{t}))^{-1} E_{0}^{m}\left(\phi\left(Y_{t}\right)\right)\right\} .
\end{aligned}
$$

To get an estimate on $G(\phi, \underline{t})$ we need some well-known facts concerning the density of the symmetric stable process $Y_{t}$.

$$
\begin{aligned}
& \text { Under } P_{0}^{0}, Y_{1} \text { has a smooth symmetric density } p(y)=p_{1}(|y|) \\
& \text { where } p_{1} \text { is decreasing and satisfies } p_{1}(r) \leqslant c^{(1)}(1+r)^{-(d+\alpha)} .
\end{aligned}
$$

This follows easily from the fact that $Y_{t} \equiv B\left(\tau_{t}\right)$, where $B$ is a $d$-dimensional Brownian motion and $\tau_{t}$ is an independent stable subordinator of index $\alpha / 2$, and well-known estimates on the distribution of $\tau_{t}$ (see Feller [1966, pp. 548, 335], Hawkes [1971, Lemma 1], Iscoe [1986, Lemma 2.2]). 
Notation. If $a>0$, let

$$
\begin{aligned}
& \psi_{d, \alpha}(a)= \begin{cases}a^{\alpha} & \text { if } d>\alpha, \\
a^{\alpha} \log ^{+} 1 / a & \text { if } d=\alpha,\end{cases} \\
& \phi_{d, \alpha}(a)=\psi_{d, \alpha}(a) \log ^{+}\left(\log ^{+} 1 / a\right) .
\end{aligned}
$$

LeMmA 2.7. $\exists c^{(2)}$ such that if $a>0$ and $\underline{t} \in T$ satisfy $2 \mu^{-1} \leqslant a^{\alpha} \leqslant 2^{-\alpha} \wedge \underline{t}^{-1}$, then

$$
G\left(I_{B(x, a)}, \underline{t}\right) \leqslant c^{(2)} \psi_{d, \alpha}(a) \quad \text { for all } x \in \mathbf{R}^{2} .
$$

Proof.

$$
\begin{aligned}
G\left(I_{B(x, a)}, \underline{t}\right) & =\int_{[0, t]} \sup _{y} P_{0}^{y}\left(\left|Y\left(\left(\underline{s}-\mu^{-1}\right)^{+}\right)-x\right| \leqslant a\right) d \lambda(\underline{s}) \\
& =\int_{[0, t]} P_{0}^{0}\left(\left|Y_{1}\right| \leqslant a\left(\left(\underline{s}-\mu^{-1}\right)^{+}\right)^{-1 / \alpha}\right) d \lambda(\underline{s}) \quad \text { (by (2.4) and scaling) } \\
& \leqslant c_{1} \int_{[0, t]}\left[a^{d}\left(\left(\underline{s}-\mu^{-1}\right)^{+}\right)^{-d / \alpha}\right] \wedge 1 d \lambda(\underline{s}) \quad(\text { by }(2.4)) \\
& \leqslant c_{1}\left[a^{\alpha}+2 \mu^{-1}+\int_{a^{\alpha}-\mu^{-1}}^{a^{-\alpha}} a^{d} s^{-d / \alpha} d s\right] \\
& \leqslant c^{(2)} \psi_{d, \alpha}(a)
\end{aligned}
$$

where we have used the restrictions on $a$ and $t$ in the last line.

We finish this section with a fundamental limit theorem that connects the system of branching stable processes $N^{(\mu)}$ with the branching diffusion $X_{t}$. As in the introduction, $Q^{m}$ denotes the law of $X$, starting at $m \in M_{F}$, on $C\left([0, \infty), M_{F}\right.$ ) (or on $\left.D\left([0, \infty), M_{F}\right)\right)$.

THEOREM 2.8. Let $m \in M_{F}$ and $m^{(\mu)} \in M_{F}^{\mu}$ satisfy $m^{(\mu)} \stackrel{w}{\rightarrow} m$ as $\mu(\in \mathbf{N}) \rightarrow \infty$.

$$
P^{m^{(\mu)}}(N \in \cdot) \stackrel{w}{\rightarrow} Q^{m}(\cdot) \quad \text { on } D\left([0, \infty), M_{F}\right) \text { as } \mu \rightarrow \infty .
$$

If $\alpha=2$, the convergence of the finite-dimensional distributions is proved in Watanabe [1968, Theorem 4.1]. Slightly different limit theorems are proved in Dawson [1975] and Roelly-Coppoletta [1986]. The martingale approach followed in the latter nicely handles tightness. In fact it is easy to modify that proof to obtain Theorem 2.8. Alternatively, the arguments in Walsh [1986] show how to prove the result using our labelling system. In any case, the result is so similar to several theorems in the literature that we have decided not to add yet another proof here.

3. Probability estimates for the upper bound on $X_{t}$. Throughout this section $\mu \in \mathbf{N}$ and $m=\mu^{-1} \sum_{i=1}^{K_{0}} \delta_{x_{i}} \in M_{F}^{\mu}$ are fixed. Dependence on these parameters is usually suppressed. In particular we write $P$ and $E$ for $P^{m}$ and $E^{m}$, respectively. 
Notation. If $\underline{t} \in T, \beta \sim \underline{t}(\beta \in I), a>0, b \in[0, \underline{t})$ and $i \in\{-1, \ldots, \mu \underline{t}-1\}$, let

$$
\begin{aligned}
& Z^{\beta, i}(a)=\mu^{-1} \sum_{\gamma \sim t, \sigma(\beta ; \gamma)=i} I\left(\left|N_{t}^{\beta}-N_{t}^{\gamma}\right| \leqslant a, N_{t}^{\gamma} \neq \Delta, N_{t}^{\beta} \neq \Delta\right), \\
& \begin{aligned}
Z^{\beta}(a, b) & =\sum_{-1 \leqslant i \leqslant \mu b} Z^{\beta, i}(a) \\
& =\mu^{-1} \sum_{\gamma \sim t, \sigma(\beta ; \gamma) \leqslant \mu b} I\left(\left|N_{t}^{\gamma}-N_{t}^{\beta}\right| \leqslant a, N_{\underline{t}}^{\gamma} \neq \Delta, N_{t}^{\beta} \neq \Delta\right),
\end{aligned} \\
& S^{\beta, i}=\{\gamma|k:| \gamma|=| \beta \mid, \sigma(\beta ; \gamma)=i, k \geqslant \mu t-i\}, \\
& S^{\beta}(b)=\{\gamma|k:| \gamma|=| \beta|, \sigma(\beta ; \gamma) \leqslant \mu b,| \beta|-[\mu b]-1 \leqslant k \leqslant| \beta \mid\}, \\
& \hat{S}^{\beta}(b)=\{\gamma:|\gamma|=|\beta|, \sigma(\beta ; \gamma)>\mu b\}, \\
& \mathscr{F}_{1}(\beta)=\sigma\left(\left\{N_{t}^{\beta} \neq \Delta\right\}\right), \quad \mathscr{F}(\beta)=\mathscr{F}(\{\beta\}) .
\end{aligned}
$$

The following result is transparent if one draws a picture of the system of branching stable processes.

LEMMA 3.1. Let $\beta \in I, \underline{t}=|\beta| / \mu, b \in[0, \underline{t})$ and $a>0$.

(a) $\left\{Z^{\beta, i}(a): i=-1, \ldots,|\beta|-1\right\}$ are conditionally independent given $\mathscr{F}(\beta)$.

(b) If $i \leqslant \mu b, Z^{\beta, i}(a)$ is $\mathscr{G}\left(S^{\beta}(b)\right) \vee \mathscr{F}_{1}(\beta)$-measurable and hence so is $Z^{\beta}(a, c)$ for any $c \leqslant b$.

(c) If $\sigma(\beta ; \gamma)>\mu b$ and $c \leqslant b$, then $Z^{\gamma}(a, c)$ is $\mathscr{F}\left(\hat{S}^{\beta}(b)\right)$-measurable and $\mathscr{F}(\gamma) \subset \mathscr{F}\left(\hat{S}^{\beta}(b)\right)$.

(d) If $A \in \mathscr{G}\left(S^{\beta}(b)\right) \vee \mathscr{F}_{1}(\beta)$ then

$$
P\left(A \mid \mathscr{F}\left(\hat{S}^{\beta}(b)\right) \vee \mathscr{F}_{1}(\beta)\right)=P\left(A \mid N_{t}^{\beta} \neq \Delta\right) \text { a.s. on }\left\{N_{\underline{t}}^{\beta} \neq \Delta\right\} \text {. }
$$

Proof. (a) If $i \in\{-1, \ldots,|\beta|-1\}$, then

$$
Z^{\beta, i}(a)=H_{a}^{\beta, i}\left(N_{t-i^{+} / \mu}^{\beta}-N_{!}^{\beta}, \omega\right) I\left(N_{t}^{\beta} \neq \Delta\right),
$$

where

$$
\begin{aligned}
& H_{a}^{\beta, i}(y, \omega) \\
& =\mu^{-1} \sum_{\gamma \sim t, \sigma(\beta ; \gamma)=i} I\left(\left|y+\sum_{k=\mu t-i}^{\mu t-1} \int I\left(\frac{k}{\mu} \leqslant s<\frac{k+1}{\mu}\right) d Y^{\gamma \mid k}(s)\right| \leqslant a\right) \\
& \quad \times I\left(e^{\gamma \mid k}=2, k=\mu t-i, \ldots, \mu \underline{t}-1\right)
\end{aligned}
$$

is $\mathscr{B}\left(R^{d}\right) \times \mathscr{G}\left(S^{\beta, i}\right)$-measurable. Hence $Z^{\beta, i}(a)$ is $\mathscr{G}\left(S^{\beta, i}\right) \vee \mathscr{F}(\beta)$-measurable. $\left\{S^{\beta, i}: \quad i=-1, \ldots,|\beta|-1\right\}$ are disjoint sets whose union is disjoint from $\{\beta|k: k \leqslant| \beta \mid\}$. It follows that $\left\{Z^{\beta, i}(a): i=-1, \ldots,|\beta|-1\right\}$ are conditionally independent given $\mathscr{F}(\beta)$.

(b) This is clear from (3.1) and (3.2).

(c) Assume $|\gamma|=|\beta|$ and $\sigma(\beta ; \gamma)>\mu b$. Then $\mathscr{F}(\gamma) \subset \mathscr{F}\left(\hat{S}^{\beta}(b)\right)$ because $\gamma \in \hat{S}^{\beta}(b)$. If $\sigma\left(\gamma ; \gamma^{\prime}\right) \leqslant \mu b$, then $\sigma\left(\beta, \gamma^{\prime}\right)>\mu b$ and so $\gamma^{\prime} \in \hat{S}^{\beta}(b)$. This shows that $\mathscr{G}\left(S^{\gamma}(b)\right) \subset \mathscr{F}\left(\hat{S}^{\beta}(b)\right)$ and therefore

$$
\mathscr{F}(\gamma) \vee \mathscr{G}\left(S^{\gamma}(b)\right) \subset \mathscr{F}(\gamma) \vee \mathscr{F}\left(\hat{S}^{\beta}(b)\right)=\mathscr{F}\left(\hat{S}^{\beta}(b)\right) .
$$

The result now follows from (b). 
(d) Let $A \in \mathscr{G}\left(S^{\beta}(b)\right)$ and $B \in \mathscr{F}\left(\hat{S}^{\beta}(b)\right)$. We may assume $x_{\beta_{0}} \neq \Delta$. Then

$$
\begin{aligned}
& \int I\left(B, N_{t}^{\beta} \neq \Delta\right) P\left(A \mid N_{t}^{\beta} \neq \Delta\right) d P \\
& \quad=P\left(B, N_{t}^{\beta} \neq \Delta\right) P\left(A, N_{t}^{\beta} \neq \Delta\right) P\left(N_{t}^{\beta} \neq \Delta\right)^{-1} \\
& \quad=P\left(B, e^{\beta \mid k}=2 \text { for } 0 \leqslant k<|\beta|\right) P\left(A, e^{\beta \mid k}=2 \text { for } 0 \leqslant k<|\beta|\right) 2^{|\beta|} .
\end{aligned}
$$

Note that if $\tilde{S}^{\beta}(b)=\left\{\gamma\left|k: \gamma \in \hat{S}^{\beta}(b), k \leqslant\right| \beta \mid\right\}$, then $\tilde{S}^{\beta}(b) \cap S^{\beta}(b)=\varnothing$ and so $\mathscr{F}\left(\hat{S}^{\beta}(b)\right)=\mathscr{G}\left(\tilde{S}^{\beta}(b)\right) \vee \sigma\left(\pi^{1}\right)$ and $\mathscr{G}\left(S^{\beta}(b)\right)$ are independent. Also $\beta \mid k \in S^{\beta}(b)$ if $|\beta|-([\mu b]+1) \leqslant k$, and $\beta \mid k \in \tilde{S}^{\beta}(b)$ if $k<|\beta|-([\mu b]+1)$. Therefore (3.3) equals

$$
\begin{aligned}
P(B, & \left.e^{\beta \mid k}=2 \text { for } 0 \leqslant k<|\beta|-([\mu b]+1)\right) 2^{-([\mu b]+1)} \\
& \times P\left(A, e^{\beta \mid k}=2 \text { for }|\beta|-([\mu b]+1) \leqslant k<|\beta|\right) 2^{-(|\beta|-([\mu b]+1))} 2^{|\beta|} \\
= & P\left(A, B, e^{\beta \mid k}=2 \text { for } 0 \leqslant k<|\beta|\right) \\
= & P\left(A, B, N_{t}^{\beta} \neq \Delta\right) .
\end{aligned}
$$

Lemma 3.2. Let $c^{(3)}=\left(2 c^{(2)}\right)^{-1}$. Assume $\beta \in I, \underline{t}=|\beta| / \mu$ and $a>0$ satisfy $2 \mu^{-1} \leqslant a^{\alpha}<\underline{t}^{-1} \wedge 3^{-\alpha}$. Then

(a) $E\left(\exp \left(\theta Z^{\beta, i}(a)\right) \mid \mathscr{F}(\beta)\right) \leqslant 1+2 \theta \mu^{-1} \mathrm{P}_{0}^{0}\left(\left|Y\left(i^{+} / \mu\right)\right| \leqslant a\right)$ a.s. on $\left\{N_{t}^{\beta} \neq \Delta\right\}$ for $0 \leqslant \theta \leqslant c^{(3)} \psi_{d, \alpha}(a)^{-1}$ and $i \in\{-1,0, \ldots,|\beta|-1\}$,

(b) $P\left(Z^{\beta}\left(a, \underline{t}-\mu^{-1}\right) \geqslant K \phi_{d, \alpha}(a) \mid \mathscr{F}(\beta)\right) \leqslant e \exp \left\{-c^{(3)} K \log \log 1 / a\right\} I\left(N_{\underline{t}}^{\beta} \neq \Delta\right)$ a.s. for any $K>0$.

Proof. (a) Fix $\beta, \underline{t}$ and $a$ as above and let $i \in\{0,1, \ldots,|\beta|-1\}$. Let $\tilde{\beta}$ be the unique multi-index of length $\mu \underline{t}-i$ such that $\tilde{\beta}|\mu \underline{t}-i-1=\beta| \mu \underline{t}-i-1$ but $\tilde{\beta} \neq \beta \mid \mu \underline{t}-i$, and for $\gamma \in\{0,1\}^{i}$ let $\tilde{\beta} \vee \gamma$ denote the multi-index of length $\mu \underline{\underline{t}}$ obtained by adding the components of $\gamma$ to $\tilde{\beta}$. For $y \in \mathbf{R}^{d}$ fixed, $H_{a}^{\beta, i}(y, \cdot)$ (given by (3.2)) is independent of $\mathscr{F}(\beta)$ and hence on $\left\{N_{t}^{\beta} \neq \Delta\right\}$ we have

$$
\begin{aligned}
E\left(\exp \left(\theta H_{a}^{\beta, i}(y, \omega)\right) \mid \mathscr{F}(\beta)\right) & \\
= & E\left(\exp \left(\theta H_{a}^{\beta, i}(y, \omega)\right)\right) \\
= & E\left(\operatorname { e x p } \left\{\theta_{\mu^{-1}} \sum_{\gamma \in\{0,1\}^{i}} I\left(\mid y+\sum_{k=0}^{i-1} \int I\left(\frac{k}{\mu} \leqslant s<\frac{k+1}{\mu}\right) d Y^{\tilde{\beta} \vee(\gamma \mid k)}\left(s+\underline{t}-\frac{i}{\mu}\right)\right)\right.\right. \\
& \left.\left.\left.\leqslant a, e^{\bar{\beta} \vee(\gamma \mid k)}=2, k=0, \ldots, i-1\right)\right\}\right) \\
= & E^{\mu^{-1} \delta_{y}}\left(\exp \left\{\theta N_{i / \mu}(B(0, a))\right\}\right) .
\end{aligned}
$$

Use Proposition 2.6(b) and Lemma 2.7 to conclude that if $\theta \in\left[0,\left(2 c^{(2)}\right)^{-1} \psi_{d, \alpha}(a)^{-1}\right]$, then

$$
\begin{aligned}
E\left(\exp \left(\theta H_{a}^{\beta, i}(y, \omega)\right) \mid \mathscr{F}(\beta)\right) & \leqslant 1+\theta \mu^{-1} 2 P_{0}^{y}\left(\left|Y_{i / \mu}\right| \leqslant a\right) \\
& \leqslant 1+\theta \mu^{-1} 2 P_{0}^{0}\left(\left|Y_{i / \mu}\right| \leqslant a\right) \quad(\text { by }(2.4)) .
\end{aligned}
$$


Now set $y=N_{t-i / \mu}^{\beta}-N_{t}^{\beta}$ and use (3.1) to prove (a) for $i \geqslant 0$. If $i=0$ we get $e^{\theta / \mu} \leqslant 1+2 \theta / \mu$ and this gives the result for $i=-1$.

(b) Assume $\underline{t}$ and $a$ are as above and let $\theta=\left(2 c^{(2)}\right)^{-1} \psi_{d, \alpha}(a)^{-1}$. Then $E\left(\exp \left(\theta Z^{\beta}\left(a, \underline{t}-\mu^{-1}\right)\right) \mid \mathscr{F}(\beta)\right)=\prod_{i=-1}^{\mu \underline{t}-1} E\left(\exp \left(\theta Z^{\beta, i}(a)\right) \mid \mathscr{F}(\beta)\right)$

(Lemma 3.1(a))

$$
\begin{aligned}
& \leqslant \exp \left\{2 \theta \int_{[0, \underline{t}]} P_{0}^{0}\left(\left|Y\left(\left(\underline{s}-\mu^{-1}\right)^{+}\right)\right| \leqslant a\right) d \lambda(\underline{s})\right\} \\
& \quad \text { (by (a)) } \\
& =\exp \left\{2 \theta G\left(I_{B(0, a)}, \underline{t}\right)\right\} \\
& \leqslant e \quad(\operatorname{Lemma} 2.7) .
\end{aligned}
$$

Chebychev's inequality implies

$$
\begin{aligned}
P\left(Z^{\beta}\left(a, \underline{t}-\mu^{-1}\right)\right. & \left.\geqslant K \phi_{d, \alpha}(a) \mid \mathscr{F}(\beta)\right) \\
& \leqslant \exp \left\{-K\left(2 c^{(2)}\right)^{-1} \log \log 1 / a\right\} e \quad(a<1 / 3) .
\end{aligned}
$$

Definition. If $b>0$, a $p$-tuple $(p \geqslant 2)$ of multi-indices, $\left(\beta_{1}, \ldots, \beta_{p}\right)$, all of the same length, is a $b$-good $p$-tuple iff $\sigma\left(\left\{\beta_{i}: i \neq j\right\} ; \beta_{j}\right) \leqslant \mu b$ for all $j \leqslant p$. $\sum_{\beta_{1}, \ldots, \beta_{p}}^{b} \sim \underline{t}$ denotes summation over all $b$-good $p$-tuples of length $\mu \underline{t}$.

LEMMA 3.3. Let $\underline{t} \in T, 2 \mu^{-1} \leqslant b<\underline{t}$, and $p \in \mathbf{N}$. Then

$$
\mu^{-2 p} \sum_{\beta_{1}, \ldots, \beta_{2 p} \sim \underline{t}}^{b} P\left(N_{t}^{\beta_{i}} \neq \Delta, i=1, \ldots, 2 p\right) \leqslant 32^{p} b^{p} p ! E\left(N_{t}\left(R^{d}\right)^{p}\right) .
$$

Proof. Fix $b \geqslant 2 \mu^{-1}$ and $\underline{t} \in T$ such that $b<\underline{t}$.

If $p \geqslant 2$ and $\left(\beta_{1}, \ldots, \beta_{p}\right)$ is a good (i.e. $b$-good) $p$-tuple we claim there are disjoint subsets $S^{(1)}$ and $S^{(2)}$ of $\{1, \ldots, p\}$ with cardinalities $[p / 2]$ and $p-[p / 2]$, respectively, such that $\sigma\left(S^{(1)} ; \beta_{j}\right) \leqslant \mu b$ for each $j \in S^{(2)}$. We proceed by induction on $p \geqslant 2$. If $p=2$ this is obvious and if $p=3$ a short argument proves the claim. Consider a good $p$-tuple $\left(\beta_{1}, \ldots, \beta_{p}\right)$ where $p \geqslant 4$ and suppose the claim holds for good $k$-tuples when $k<p$. Choose $j \leqslant p-1$ such that $\sigma\left(\beta_{j} ; \beta_{p}\right) \leqslant \mu b$.

Case 1. $\sigma\left(\left\{\beta_{i}: i \neq j\right.\right.$ or $\left.\left.p\right\} ; \beta_{j}\right)>\mu b$.

We first show

$$
\left(\beta_{1}, \ldots, \beta_{j-1}, \beta_{j+1}, \ldots, \beta_{p-1}\right) \text { is a } \operatorname{good}(p-2) \text {-tuple. }
$$

Let $i \in\{1, \ldots, p-1\}-\{j\}$. If $\sigma\left(\beta_{p} ; \beta_{i}\right) \leqslant \mu b$, then $\sigma\left(\beta_{j} ; \beta_{p}\right) \leqslant \mu b$ would imply $\sigma\left(\beta_{j} ; \beta_{i}\right) \leqslant \mu b$, which contradicts our assumption. Therefore $\sigma\left(\beta_{p} ; \beta_{i}\right)>\mu b$. One also has $\sigma\left(\beta_{j} ; \beta_{i}\right)>\mu b$ by assumption. Recalling that $\left(\beta_{1}, \ldots, \beta_{p}\right)$ is a good $p$-tuple, one concludes $\sigma\left(\left\{\beta_{k}: k \neq j\right.\right.$ or $\left.\left.p\right\} ; \beta_{i}\right) \leqslant \mu b$ and hence obtains (3.4).

By induction there are disjoint subsets $\bar{S}^{(1)}$ and $\bar{S}^{(2)}$ of $\{1, \ldots, p-1\}-\{j\}$ with cardinalities $[p / 2]-1$ and $p-1-[p / 2]$, respectively, such that $\forall k \in \bar{S}^{(2)}$, $\sigma\left(\bar{S}^{(1)} ; \beta_{k}\right) \leqslant \mu b$. It is now easy to see $S^{(1)}=\bar{S}^{(1)} \cup\left\{\beta_{j}\right\}$ and $S^{(2)}=\bar{S}^{(2)} \cup\left\{\beta_{p}\right\}$ are the required sets (recall $\left.\sigma\left(\beta_{j} ; \beta_{p}\right) \leqslant \mu b\right)$. 
Case 2. $\sigma\left(\left\{\beta_{i}: i \neq j\right.\right.$ or $\left.\left.p\right\} ; \beta_{j}\right) \leqslant \mu b$.

We first show

$$
\left(\beta_{1}, \ldots, \beta_{p-1}\right) \text { is a good }(p-1) \text {-tuple. }
$$

Let $i \in\{1, \ldots, p-1\}$. If $i=j$ then $\sigma\left(\left\{\beta_{k}: k \neq j\right.\right.$ or $\left.\left.p\right\} ; \beta_{j}\right) \leqslant \mu b$ by assumption. Assume $i \neq j$. Choose $k \neq i, k \leqslant p$ such that $\sigma\left(\beta_{k} ; \beta_{i}\right) \leqslant \mu b\left(\right.$ recall $\left(\beta_{1}, \ldots, \beta_{p}\right)$ is a good $p$-tuple). If $k=p$ then $\sigma\left(\beta_{j} ; \beta_{p}\right) \leqslant \mu b$ implies $\sigma\left(\beta_{j} ; \beta_{i}\right) \leqslant \mu b$. Therefore we may assume $k \leqslant p-1, k \neq i$. This proves (3.5).

By induction there are disjoint subsets $\bar{S}^{(1)}$ and $\bar{S}^{(2)}$ of $\{1, \ldots, p-1\}$ with cardinalities $[(p-1) / 2]$ and $p-1-[(p-1) / 2]$, respectively, that satisfy the desired property. We know $\sigma\left(\beta_{j} ; \beta_{p}\right) \leqslant \mu b$ where $j \in \bar{S}^{(1)}$ or $j \in \bar{S}^{(2)}$. In the latter case $\sigma\left(\beta_{i} ; \beta_{j}\right) \leqslant \mu b$ for some $i \in \bar{S}^{(1)}$ and therefore $\sigma\left(\beta_{i} ; \beta_{p}\right) \leqslant \mu b$. We have shown $\sigma\left(\beta_{i} ; \beta_{p}\right) \leqslant \mu b$ for some $i \in \bar{S}^{(1)}$. It is therefore clear that

$$
\left.\left.\begin{array}{l}
S^{(1)}=\bar{S}^{(1)} \\
S^{(2)}=\bar{S}^{(2)} \cup\{p\}
\end{array}\right\} \quad \text { if } m \text { is odd; } \quad \begin{array}{l}
S^{(1)}=\bar{S}^{(1)} \cup\{p\} \\
S^{(2)}=\bar{S}^{(2)}
\end{array}\right\} \quad \text { if } m \text { is even }
$$

defines the required subsets. The induction proof is complete.

Now fix $p \in \mathbf{N}$ and a good $2 p$-tuple $\left(\beta_{1}, \ldots, \beta_{2 p}\right)\left(\beta_{j} \sim t\right)$. Let $S^{(1)}=\left\{i_{1}, \ldots, i_{p}\right\}$ and $S^{(2)}=\left\{j_{1}, \ldots, j_{p}\right\}\left(i_{k}<i_{k+1}, j_{k}<j_{k+1}\right)$ be disjoint subsets of $\{1, \ldots, 2 p\}$ that satisfy

$$
\sigma\left(\left\{\beta_{i_{k}}: k=1, \ldots, p\right\} ; \beta_{j^{\prime}}\right) \leqslant \mu b, \quad k^{\prime}=1, \ldots, p .
$$

Conversely given any two $p$-tuples $\left(\beta_{i_{1}}, \ldots, \beta_{i_{p}}\right)$ and $\left(\beta_{j_{1}}, \ldots, \beta_{j_{p}}\right)$ that satisfy (3.6), there are at most $\left(\begin{array}{c}2 p \\ p\end{array}\right)$ good $2 p$-tuples that could give rise to these $p$-tuples in the above manner, corresponding to the $\left(\begin{array}{c}2 p \\ p\end{array}\right)$ ways to choose the subset $S^{(1)}$. Therefore

$$
\begin{aligned}
& \mu^{-2 p} E\left(\sum_{\beta_{1}, \ldots, \beta_{2 p}-t}^{b} I\left(N_{t}^{\beta_{i}} \neq \Delta, i=1, \ldots, 2 p\right)\right) \\
& \leqslant\left(\begin{array}{c}
2 p \\
p
\end{array}\right) E\left(\mu^{-2 p} \sum_{\beta_{1}-t} \cdots \sum_{\beta_{p}-t} \sum_{\substack{\beta_{p+1}, \ldots, \beta_{2 p}-t \\
\left(\left\{\beta_{k}: k \leqslant p\right\} ; \beta_{j}\right) \leqslant \mu b \\
\text { for } j=p+1, \ldots, 2 p}} I\left(N_{i}^{\beta_{i}} \neq \Delta, i=1, \ldots, 2 p\right)\right)
\end{aligned}
$$

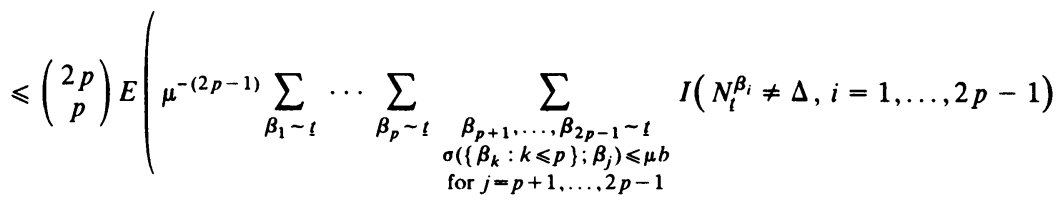

$$
\begin{aligned}
& \times E\left(\sum_{\substack{\beta_{2 p}-t \\
\sigma\left(\left\{\beta_{i}: i \leqslant 2 p-1\right\} ; \beta_{2 p}\right) \leqslant \mu b}} I\left(N_{t}^{\left.\left.\beta_{2 p} \neq \Delta\right) \mid \mathscr{F}\left(\beta_{1}, \ldots, \beta_{2 p-1}\right)\right)}\right) .\right.
\end{aligned}
$$


The last conditional expectation is bounded by $(2 p-1)([\mu b]+2) / \mu$ (apply Lemma $2.4(c)$ with $\phi=1$ ). Proceeding inductively, we can bound the above sum by

$$
\begin{aligned}
\frac{(2 p) !}{p ! p !}(2 p-1) & \cdots p\left(\frac{[\mu b]+2}{\mu}\right)^{p} E\left(N_{t}\left(\mathbf{R}^{d}\right)^{p}\right) \\
\leqslant & 2^{2 p} p ! 2^{2 p}(b+2 / \mu)^{p} E\left(N_{t}\left(\mathbf{R}^{d}\right)^{p}\right) \\
& \leqslant 32^{p} b^{p} p ! E\left(N_{t}\left(\mathbf{R}^{d}\right)^{p}\right) \quad(b \geqslant 2 / \mu) .
\end{aligned}
$$

Let $\delta \in(0,2)$ be fixed throughout the rest of this section.

Proposition 3.4. Assume $K>2 / c^{(3)}, a^{\alpha} \wedge a^{\delta} \geqslant 2 \mu^{-1}, M \in \mathbf{N}, M \geqslant m\left(\mathbf{R}^{d}\right)$, and $\underline{t} \in\left[M^{-1}, M\right] \cap T$. There is an $a_{0}=a_{0}(\delta, K, M)>0$ such that if $a \leqslant a_{0}$, then

$$
P\left(\mu^{-1} \sum_{\beta \sim t} I\left(Z^{\beta}\left(a, a^{\delta}\right) \geqslant K \phi_{d, \alpha}(a)\right) \geqslant \frac{1}{2}\left(\log \frac{1}{a}\right)^{-K c^{(3)} / 2}\right) \leqslant a^{K} .
$$

Proof. Fix $K, a, M$ and $t$ as in the first line of the proposition, assume also $a^{\delta}<M^{-1}$, and let $p \in \mathbf{N}$. Define

$$
\begin{aligned}
F(\underline{t}, a, K)=\frac{1}{\mu} \sum_{\beta \sim t}( & I\left(Z^{\beta}\left(a, a^{\delta}\right) \geqslant K \phi_{d, \alpha}(a)\right) \\
& \left.-P\left(Z^{\beta}\left(a, a^{\delta}\right) \geqslant K \phi_{d, \alpha}(a) \mid \mathscr{F}_{1}(\beta)\right)\right) .
\end{aligned}
$$

Then

$$
\begin{aligned}
& E\left(F(t, a, K)^{2 p}\right) \\
&=\mu^{-2 p} \sum_{\beta_{1} \sim t} \cdots \sum_{\beta_{2 p} \sim t} E\left(\prod _ { i = 1 } ^ { 2 p } \left(I\left(Z^{\beta_{i}}\left(a, a^{\delta}\right) \geqslant K \phi_{d, \alpha}(a)\right)\right.\right. \\
&\left.\left.-P\left(Z^{\beta_{i}}\left(a, a^{\delta}\right) \geqslant K \phi_{d, \alpha}(a) \mid \mathscr{F}_{1}\left(\beta_{i}\right)\right)\right)\right) .
\end{aligned}
$$

Suppose $\beta_{1}, \ldots, \beta_{2 p} \sim \underline{t}$ satisfy $\sigma\left(\left\{\beta_{j}: j \leqslant 2 p, j \neq i\right\} ; \beta_{i}\right)>\mu a^{\delta}$ for some $i \leqslant 2 p$. To simplify the notation, assume $i=2 p$. Then

$$
\begin{aligned}
E\left(\prod_{j=1}^{2 p}\left(I\left(Z^{\beta_{j}}\left(a, a^{\delta}\right) \geqslant K \phi_{d, \alpha}(a)\right)-P\left(Z^{\beta_{j}}\left(a, a^{\delta}\right) \geqslant K \phi_{d, \alpha}(a) \mid \mathscr{F}_{1}\left(\beta_{j}\right)\right)\right)\right) \\
=E\left[\prod_{j=1}^{2 p-1}\left(I\left(Z^{\beta_{j}}\left(a, a^{\delta}\right) \geqslant K \phi_{d, \alpha}(a)\right)-P\left(Z^{\beta_{j}}\left(a, a^{\delta}\right) \geqslant K \phi_{d, \alpha}(a) \mid \mathscr{F}_{1}\left(\beta_{j}\right)\right)\right)\right. \\
\times E\left(\left(I\left(Z^{\beta_{2 p}}\left(a, a^{\delta}\right) \geqslant K \phi_{d, \alpha}(a)\right)-P\left(Z^{\beta_{2 p}}\left(a, a^{\delta}\right) \geqslant K \phi_{d, \alpha}(a) \mid \mathscr{F}_{1}\left(\beta_{2 p}\right)\right)\right)\right. \\
\left.\left.\mid \mathscr{F}\left(\hat{S}^{\beta_{2 p}}\left(a^{\delta}\right)\right) \vee \mathscr{F}_{1}\left(\beta_{2 p}\right)\right)\right]
\end{aligned}
$$


The last conditional expectation is zero by Lemma 3.1(b), (d). Therefore (3.7) implies

$$
\begin{aligned}
E\left(F(t, a, K)^{2 p}\right) \leqslant \mu^{-2 p} \sum_{\beta_{1}, \ldots, \beta_{2 p} \sim t}^{a^{\delta}} P\left(N_{t}^{\beta_{j}} \neq \Delta, j=1, \ldots, 2 p\right) \\
\leqslant 32^{p} p ! a^{\delta p} E\left(N_{t}\left(\mathbf{R}^{d}\right)^{p}\right) \quad(\text { Lemma 3.3) } \\
\leqslant c(p, M) a^{\delta p} \quad\left(\text { Proposition 2.6(c) and } m\left(\mathbf{R}^{d}\right) \vee \underline{t} \leqslant M\right) .
\end{aligned}
$$

If $a<1 / 3 \wedge M^{-1 / \alpha}$, and $\lambda=\frac{1}{2}(\log 1 / a)^{-K c^{(3)} / 2}$, then

$$
\begin{aligned}
& P\left(\mu^{-1} \sum_{\beta \sim t} I\left(Z^{\beta}\left(a, a^{\delta}\right) \geqslant K \phi_{d, \alpha}(a)\right) \geqslant \lambda\right) \\
& \leqslant P\left(F(\underline{t}, a, K) \geqslant \frac{\lambda}{2}\right)+P\left(\mu^{-1} \sum_{\beta \sim t} P\left(Z^{\beta}\left(a, \underline{t}-\mu^{-1}\right) \geqslant K \phi_{d, \alpha}(a) \mid \mathscr{F}_{1}(\beta)\right) \geqslant \frac{\lambda}{2}\right) \\
& \leqslant 16^{p}\left(\log \frac{1}{a}\right)^{p K c^{(3)}} c(p, M) a^{\delta p}+P\left(e \exp \left\{-c^{(3)} K \log \log \frac{1}{a}\right\} N_{t}\left(\mathbf{R}^{d}\right) \geqslant \frac{\lambda}{2}\right)
\end{aligned}
$$

(by (3.8) and Lemma 3.2(b))

$$
\begin{aligned}
& \leqslant c_{1}(p, M)\left(\log \frac{1}{a}\right)^{p K c^{(3)}} a^{\delta p}+P\left(N_{t}\left(\mathbf{R}^{d}\right) \geqslant(4 e)^{-1}\left(\log \frac{1}{a}\right)^{K c^{(3)} / 2}\right) \\
& \leqslant c_{1}(p, M)\left(\log \frac{1}{a}\right)^{p K c^{(3)}} a^{\delta p}+e^{M m\left(\mathbf{R}^{d}\right)} \exp \left\{-(16 e M)^{-1}\left(\log \frac{1}{a}\right)^{K c^{(3)} / 2}\right\}
\end{aligned}
$$

$$
\text { (Proposition 2.6(c) with } \left.\theta=\left(2\left(\underline{t}+\mu^{-1}\right)\right)^{-1}\right) \text {. }
$$

Choose $p>K \delta^{-1}$ and then $a_{0}=a_{0}(\delta, K, M)$ small enough so that the above expression is less than $a^{K}$ if $a<a_{0}$ (recall $K c^{(3)} / 2>1$ ).

We have found a good probabilistic bound on $Z^{\beta}\left(a, a^{\delta}\right)$ which represents the contribution to $N_{t}\left(B\left(N_{t}^{\beta}, a\right)\right)$ by "close cousins." This is the major portion of $N_{t}\left(B\left(N_{!}^{\beta}, a\right)\right)$ and the impatient (or exhausted) reader may want to skip to the next section. If $d>\alpha+2$, a simple first moment argument can handle the contribution from "distant cousins" (see Theorem 3.8). To handle $\alpha \leqslant d \leqslant \alpha+2$ a more complicated argument seems to be needed.

Notation. If $\beta \in I, \beta \sim \underline{t} \in T$ and $a>0$, let

$$
\begin{aligned}
W^{\beta}(a)= & Z^{\beta}(a, \underline{t})-Z^{\beta}\left(a, a^{\delta}\right) \\
& =\mu^{-1} \sum_{\gamma \sim t, a^{\delta} \mu<\sigma(\beta ; \gamma) \leqslant \underline{t}} I\left(\left|N_{t}^{\gamma}-N_{t}^{\beta}\right| \leqslant a, N_{t}^{\gamma} \neq \Delta, N_{t}^{\beta} \neq \Delta\right), \\
W_{\infty}^{\beta}(a) & =Z^{\beta}(a, \underline{t})-Z^{\beta}\left(a, \underline{t}-\mu^{-1}\right) \\
& =\mu^{-1} \sum_{\gamma \sim t, \sigma(\beta ; \gamma)=\mu \underline{t}} I\left(\left|N_{t}^{\gamma}-N_{t}^{\beta}\right| \leqslant a, N_{t}^{\gamma} \neq \Delta, N_{t}^{\beta} \neq \Delta\right) .
\end{aligned}
$$


LEMMA 3.5. If $p, M \in \mathbf{N}$, there is a $c^{(4)}(M, p)$ such that

$$
\begin{aligned}
& E\left(\left(\mu^{-1} \sum_{\beta \sim t} W_{\infty}^{\beta}(a)\right)^{p}\right) \\
& \leqslant c^{(4)}(M, p)\left(m\left(\mathbf{R}^{d}\right)+1\right)^{2 p}\left(\left(a \underline{t}^{-1 / \alpha}\right)^{d}+a^{2 \alpha}(1+\log 1 / a)\right)^{p}
\end{aligned}
$$

whenever $a^{\alpha} \in[4 / \mu, 1], \underline{t} \in[6 / \mu, M] \cap T$.

Proof. Let $M, a, t$ be as above and assume $p \in \mathbf{N} \geqslant 2$.

$$
\begin{aligned}
& E\left(\left(\mu^{-1} \sum_{\beta \sim t} W_{\infty}^{\beta}(a)\right)^{p}\right) \\
&=E\left(\sum_{\substack{\mu_{1}, \ldots, \beta_{p-1} \sim t, \sigma\left(\beta_{i}, \gamma_{i}\right)=\mu t \\
\mu_{1}, \ldots, \gamma_{p-1} \sim t}} I\left(\left|N_{t}^{\gamma_{i}}-N_{l}^{\beta_{i}}\right| \leqslant a, i=1, \ldots, p-1\right)\right. \\
&\left.\times\left(\sum_{1}^{\left(\beta_{i}, \gamma_{i} ; i<p\right)}+\sum_{2}^{\left(\beta_{i}, \gamma_{i} ; i<p\right)}\right)\right),
\end{aligned}
$$

where

$$
\sum_{k}^{\left(\beta_{i}, \gamma_{i} ; i<p\right)}=\mu^{-2} \sum_{\substack{\beta_{p}, \gamma_{p} \sim t \\ \sigma\left(\beta_{p} ; \gamma_{p}\right)=\mu t}}^{(k)} P\left(\left|N_{\underline{t}}^{\beta_{p}}-N_{\underline{t}}^{\gamma_{p}}\right| \leqslant a \mid \mathscr{F}\left(\beta_{i}, \gamma_{i}: i<p\right)\right), \quad k=1,2 .
$$

Here $\Sigma^{(1)}$ indicates the summation is over those $\left(\beta_{p}, \gamma_{p}\right)$ for which

$$
\sigma\left(\left\{\beta_{i}, \gamma_{i}: i<p\right\} ; \beta_{p}\right)<\mu \underline{t} \text { and } \sigma\left(\left\{\beta_{i}, \gamma_{i}: i<p\right\} ; \gamma_{p}\right)<\mu \underline{t} \text {, }
$$

and $\Sigma^{(2)}$ indicates the summation over those $\left(\beta_{p}, \gamma_{p}\right)$ for which

$$
\sigma\left(\left\{\beta_{i}, \gamma_{i}: i<p\right\} ; \beta_{p}\right)=\mu \underline{t} \text { or } \sigma\left(\left\{\beta_{i}, \gamma_{i}: i<p\right\} ; \gamma_{p}\right)=\mu \underline{t} \text {. }
$$

\section{By symmetry}

$$
\begin{aligned}
& \sum_{2}^{\left(\beta_{i}, \gamma_{i} ; i<p\right)} \leqslant 2 \mu^{-2} \sum_{\beta_{p}, \gamma_{p} \sim t ; \sigma\left(\left\{\beta_{i}, \gamma_{j}: i \leqslant p, j<p\right\} ; \gamma_{p}\right)=\mu t} E\left(P \left(\left|N_{t}^{\beta_{p}}-N_{t}^{\gamma_{p}}\right| \leqslant a \mid\right.\right. \\
& \left.\left.\mathscr{F}\left(\beta_{i}, \gamma_{j}: i \leqslant p, j<p\right)\right) \mid \mathscr{F}\left(\beta_{i}, \gamma_{i}: i<p\right)\right) \\
& \leqslant 2 \mu^{-2} \sum_{\beta_{p}, \gamma_{p}-t} E\left(P_{0}^{N_{t}^{\beta_{p}}-N_{0}^{\gamma}}(|Y(\underline{t})| \leqslant a) 2^{-\mu_{t}} I\left(N_{i}^{\beta_{p}} \neq \Delta, N_{0}^{\gamma_{p}} \neq \Delta\right) \mid \mathscr{F}\left(\beta_{i}, \gamma_{i}: i<p\right)\right) \\
& \text { (Lemma 2.5(b)) } \\
& \leqslant 2 P_{0}^{0}(|Y(t)| \leqslant a) m\left(\mathbf{R}^{d}\right) \mu^{-1}\left[\sum_{\beta_{p} \sim t, \sigma\left(\left\{\beta_{i}, \gamma_{i}: i<p\right\} ; \beta_{p}\right)<\mu t} P\left(N_{t}^{\beta_{p}} \neq \Delta \mid \mathscr{F}\left(\beta_{i}, \gamma_{i}: i<p\right)\right)\right. \\
& +\sum_{\beta_{p} \sim t, \sigma\left(\left\{\beta_{i}, \gamma_{i}: i<p\right\} ; \beta_{p}\right)=\mu t} P\left(N_{i}^{\left.\left.\beta_{p} \neq \Delta\right)\right]}\right. \\
& \leqslant c_{1} a^{d} \underline{t}^{-d / \alpha} m\left(\mathbf{R}^{d}\right)\left[2(p-1)\left(\underline{t}+\mu^{-1}\right)+m\left(\mathbf{R}^{d}\right)\right] \quad(\text { Lemma 2.4 (c)), } \\
& \sum_{2}^{\left(\beta_{i}, \gamma_{i} ; i<p\right)} \leqslant c_{2} a^{d} \underline{t}^{-d / \alpha} m\left(\mathbf{R}^{d}\right)(p-1)\left[t+m\left(\mathbf{R}^{d}\right)\right]
\end{aligned}
$$


To bound $\Sigma_{1}$, note that if $\sigma\left(\beta_{p} ; \gamma_{p}\right)=\mu t, \sigma\left(\left\{\beta_{i}, \gamma_{i}: i<p\right\} ; \beta_{p}\right)=j<\mu \underline{t}$ and $\sigma\left(\left\{\beta_{i}, \gamma_{i}: i<p\right\} ; \gamma_{p}\right)=k<\mu t$ then Lemma 2.5(b) implies

$$
\begin{aligned}
& P\left(\left|N_{t}^{\beta_{p}}-N_{i}^{\gamma_{p}}\right| \leqslant a \mid \mathscr{F}\left(\beta_{i}, \gamma_{i}: i<p\right)\right)
\end{aligned}
$$

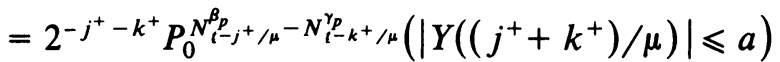

$$
\begin{aligned}
& \times I\left(N_{t-j^{+} / \mu}^{\beta_{p^{+}}} \neq \Delta, N_{t}^{\gamma_{\underline{p^{+}}} k^{+} / \mu}, \Delta\right) \\
& \leqslant 2^{-j^{+}-k^{+}} P_{0}^{0}\left(\left|Y\left(\left(j^{+}+k^{+}\right) / \mu\right)\right| \leqslant a\right) .
\end{aligned}
$$

Sum over $j, k$, noting (as in the proof of Lemma 2.4(b)) that

$$
\begin{aligned}
\operatorname{card}\left\{\left(\beta_{p}, \gamma_{p}\right): \beta_{p} \sim t\right. & \left.\underline{t} \gamma_{p} \sim \underline{t}, \sigma\left(\left\{\beta_{i}, \gamma_{i}: i<p\right\} ; \beta_{p}\right)=j, \sigma\left(\left\{\beta_{i}, \gamma_{i}: i<p\right\} ; \gamma_{p}\right)=k\right\} \\
& \leqslant(2(p-1))^{2} 2^{j^{+}+k^{+}}, \quad j, k<\mu \underline{t},
\end{aligned}
$$

to obtain

$$
\begin{aligned}
& \sum_{1}^{\left(\beta_{i}, \gamma_{i}: i<p\right)} \leqslant \mu^{-2} \sum_{-1 \leqslant j<\mu t \underline{t}} \sum_{-1 \leqslant k<\mu \underline{t}} P_{0}^{0}\left(\left|Y\left(\left(j^{+}+k^{+}\right) / \mu\right)\right| \leqslant a\right) 4(p-1)^{2} \\
& \leqslant c_{3}(p-1)^{2} \int_{[0, t]^{2}}\left[a^{d}\left((\underline{s}+\underline{u}-2 / \mu)^{+}\right)^{-d / \alpha} \wedge 1\right] d \lambda(\underline{s}) d \lambda(\underline{u}) \\
& \leqslant c_{3}(p-1)^{2} \int_{\left[0, \underline{t}+\mu^{-1}\right]^{2}}\left(a^{d}\left((s+u-4 / \mu)^{+}\right)^{-d / \alpha} \wedge 1\right) d s d u \\
& =\left(c_{3} / 2\right)(p-1)^{2}\left[\int_{0}^{t+\mu^{-1}} \int_{-v}^{v} a^{d}\left((v-4 / \mu)^{+}\right)^{-d / \alpha} \wedge 1 d w d v\right. \\
& \left.\quad+\int_{t+\mu^{-1}}^{2\left(\underline{t}+\mu^{-1}\right)} \int_{-\left(2\left(\underline{t}+\mu^{-1}\right)-v\right)}^{2\left(t+\mu^{-1}\right)-v} a^{d}\left((v-4 / \mu)^{+}\right)^{-d / \alpha} \wedge 1 d w d v\right] \\
& \leqslant \begin{cases}c_{4}(p-1)^{2}\left[a^{2 \alpha}+a^{d} \underline{t}^{2-d / \alpha}\right] & \text { if } d / \alpha \neq 2, \\
c_{4}(p-1)^{2}\left[a^{2 \alpha}+a^{2 \alpha} \log ^{+}\left(\underline{t} a^{-\alpha}\right)\right] & \text { if } d / \alpha=2,\end{cases}
\end{aligned}
$$

by a routine integration (the lower bounds on $a$ and $t$ are used here). Therefore

$$
\sum_{1}^{\left(\beta_{i}, \gamma_{i}: i<p\right)} \leqslant c_{4}(p-1)^{2}\left[a^{2 \alpha}\left(1+\left(\log \left(\underline{t} a^{-\alpha}\right)\right)^{+}\right)+a^{d} \underline{t}^{2-d / \alpha}\right] .
$$

(3.9), (3.10) and (3.11) imply for $p \geqslant 2$,

$$
\begin{aligned}
& E\left(\left(\mu^{-1} \sum_{\beta \sim t} W_{\infty}^{\beta}(a)\right)^{p}\right) / E\left(\left(\mu^{-1} \sum_{\beta \sim t} W_{\infty}^{\beta}(a)\right)^{p-1}\right) \\
& \quad \leqslant c_{5}(p-1)^{2}\left(m\left(\mathbf{R}^{d}\right)+1\right)\left[a^{d} \underline{t}^{-d / \alpha}\left(\underline{t}+m\left(\mathbf{R}^{d}\right)+\underline{t}^{2}\right)+a^{2 \alpha}\left(1+\log ^{+}\left(\underline{t} a^{-\alpha}\right)\right)\right] \\
& \quad \leqslant c_{6}(M, p)\left(m\left(\mathbf{R}^{d}\right)+1\right)^{2}\left[\left(a \underline{t} \underline{-}^{-1 / \alpha}\right)^{d}+a^{2 \alpha}(1+\log 1 / a)\right] .
\end{aligned}
$$


If $p=1$ the left side of (3.9) is easy to compute (as for Proposition 2.2) and the obvious induction therefore gives

$$
\begin{aligned}
E\left(\left(\mu^{-1} \sum_{\beta \sim t} W_{\infty}^{\beta}(a)\right)^{p}\right) \\
\leqslant c_{6}(M, p)^{p-1}\left(m\left(\mathbf{R}^{d}\right)+1\right)^{2(p-1)}\left[\left(a \underline{t}^{-1 / \alpha}\right)^{d}+a^{2 \alpha}(1+\log 1 / a)\right]^{p-1} \\
\quad \times P_{0}^{0}\left(\left|Y_{2 \underline{t}}\right| \leqslant a\right) E\left(X_{t}\left(\mathbf{R}^{d}\right)\right)^{2} \\
\leqslant c^{(4)}(M, p)\left(m\left(\mathbf{R}^{d}\right)+1\right)^{2 p}\left[\left(a \underline{t}^{-1 / \alpha}\right)^{d}+a^{2 \alpha}(1+\log 1 / a)\right]^{p}
\end{aligned}
$$

(Proposition 2.2).

If $d>\alpha$, assume $\delta>0$ satisfies

$$
d\left(1-\delta \alpha^{-1}\right)>\alpha .
$$

Proposition 3.6. Assume $d>\alpha$. $\exists \varepsilon_{0}=\varepsilon_{0}(\delta)>0$ and for each $M, p \in \mathbf{N}$ with $M \geqslant m\left(\mathbf{R}^{d}\right), \exists c^{(5)}=c^{(5)}(M, p)$ such that

$$
P\left(\mu^{-1} \sum_{\beta \sim t} I\left(W^{\beta}(a)>a^{\alpha}\right) \geqslant \lambda\right) \leqslant c^{(5)} \lambda^{-p} a^{\varepsilon_{0} p}
$$

whenever $\lambda>0, a \in[0,1], a^{\alpha} \wedge a^{\delta} \geqslant 6 \mu^{-1}$, and $\underline{t} \in\left[a^{\delta}, M\right] \cap T$.

Proof. Let $M, p, \lambda, a$ and $\underline{t}$ be as above. If $\underline{s}, \underline{u} \in T$, then

$$
\begin{aligned}
\mu^{-1} \sum_{\beta \sim \underline{s}} W_{\infty}^{\beta}(a) \circ \theta_{\underline{u}} & =\mu^{-2} \sum_{\beta \sim \underline{s}} \sum_{\gamma \sim \underline{s}, \gamma_{0} \neq \beta_{0}} I\left(\left|N_{s+\underline{u}}^{\rho_{u}(\beta)}-N_{s+\underline{u}}^{\rho_{u}(\gamma)}\right| \leqslant a\right) \quad(\text { by }(2.1)) \\
& =\mu^{-2} \sum_{\beta \sim \underline{s}+\underline{u}} \sum_{\gamma \sim \underline{s}+\underline{u}, \sigma(\beta ; \gamma) \geqslant \mu \underline{s}} I\left(\left|N_{s+\underline{u}}^{\beta}-N_{\varepsilon+\underline{u}}^{\gamma}\right| \leqslant a\right) .
\end{aligned}
$$

Let $\underline{s}=\left[a^{\delta} \mu\right] / \mu, \underline{u}=\underline{t}-\underline{s} \geqslant 0$ in the above to get

$$
\mu^{-1} \sum_{\beta \sim \underline{s}} W_{\infty}^{\beta}(a) \circ \theta_{t-\underline{s}} \geqslant \mu^{-1} \sum_{\beta \sim \underline{t}} W^{\beta}(a) .
$$

We can now apply the Markov property (Proposition 2.3(b)) and conclude

$$
\begin{aligned}
& E\left(\left(\mu^{-1} \sum_{\beta \sim t} W^{\beta}(a)\right)^{p}\right)=E\left(E^{N_{t-s}}\left(\left(\mu^{-1} \sum_{\beta \sim \underline{s}} W_{\infty}^{\beta}(a)\right)^{p}\right)\right) \\
& \leqslant c^{(4)}(M, p)\left(\left(a \underline{s}^{-1 / \alpha}\right)^{d}+a^{2 \alpha}(1+\log 1 / a)\right)^{p} E^{m}\left(\left(N_{t-s}\left(R^{d}\right)+1\right)^{2 p}\right)
\end{aligned}
$$

$$
\leqslant c_{1}(M, p)\left(a^{d(1-\delta / \alpha)}+a^{2 \alpha}(1+\log 1 / a)\right)^{p}
$$

(Proposition 2.6(c) and $m\left(\mathbf{R}^{d}\right) \vee \underline{t} \leqslant M$ )

$$
\leqslant c^{(5)}(M, p) a^{\left(\alpha+\varepsilon_{0}\right) p}
$$


for some $\varepsilon_{0}=\varepsilon_{0}(\delta)>0$, by (3.12). Therefore

$$
\begin{aligned}
P\left(\frac{1}{\mu} \sum_{\beta \sim \underline{t}} I\left(W^{\beta}(a) \geqslant a^{\alpha}\right) \geqslant \lambda\right) & \leqslant \lambda^{-p} a^{-\alpha p} E\left(\left(\mu^{-1} \sum_{\beta \sim t} W^{\beta}(a)\right)^{p}\right) \\
& \leqslant c^{(5)}(M, p) \lambda^{-p} a^{\varepsilon_{0} p} .
\end{aligned}
$$

Notation. If $a, K, t \geqslant 0$, let

$$
S(t, a, K)(\omega)=\left\{x: N_{t}(B(x, a)) \geqslant K\right\} .
$$

TheOREM 3.7. Assume $d>\alpha$. Let $M, N \in \mathrm{N} . \exists K=K(N)>0$ and $a_{1}=$ $a_{1}(M, N)>0$ such that if $m\left(\mathbf{R}^{d}\right) \leqslant M, \underline{t} \in\left[M^{-1}, M\right] \cap T, a \in\left(0, a_{1}\right]$ and $\mu \geqslant$ $6\left(a^{-\alpha} \vee a^{-\delta}\right)$, then

$$
P\left(N_{t}\left(S\left(\underline{t}, a, K \phi_{\alpha}(a)\right)\right)>(\log 1 / a)^{-2}\right) \leqslant a^{N} .
$$

Proof. Let

$$
K(N)=\max \left(N, 4 / c^{(3)}\right)+2,
$$

and

$$
a_{1}(M, N)=\min \left(e^{-e}, M^{-1 / \delta}, a_{0}(\delta, K(N)-1, M)\right)
$$

$\left(a_{0}\right.$ is as in Proposition 3.4). Choose $\underline{t} \in\left[M^{-1}, M\right] \cap T, a \in\left(0, a_{1}\right], \mu \geqslant$ $6\left(a^{-\delta} \vee a^{-\alpha}\right)$ and $p \in \mathbf{N}, p>N / \tilde{\varepsilon}_{0}(\delta)$. Then

$$
\begin{aligned}
P\left(N_{t}\left(S\left(\underline{t}, a, K \phi_{\alpha}(a)\right)\right)>(\log 1 / a)^{-2}\right) & \\
= & P\left(\mu^{-1} \sum_{\beta \sim t} I\left(Z^{\beta}\left(a, a^{\delta}\right)+W^{\beta}(a) \geqslant K \phi_{\alpha}(a)\right) \geqslant(\log 1 / a)^{-2}\right) \\
\leqslant & P\left(\mu^{-1} \sum_{\beta \sim t} I\left(Z^{\beta}\left(a, a^{\delta}\right) \geqslant(K-1) \phi_{\alpha}(a)\right) \geqslant(\log 1 / a)^{-2} / 2\right) \\
& +P\left(\mu^{-1} \sum_{\beta \sim t} I\left(W^{\beta}(a) \geqslant \phi_{\alpha}(a)\right) \geqslant(\log 1 / a)^{-2} / 2\right) \\
\leqslant & a^{K-1}+c^{(5)}(M, p) 2^{p}(\log 1 / a)^{2 p} a^{\tilde{\varepsilon}_{0} p},
\end{aligned}
$$

where we have applied Propositions 3.4 and 3.6. (The choice of $K$ implies $(\log 1 / a)^{-2} \geqslant(\log 1 / a)^{-(K-1) c^{(3)} / 2}$ so that 3.4 applies. Also $\underline{t} \geqslant M^{-1} \geqslant a^{\delta}$ so 3.6 applies.) By making $a_{1}$ smaller, if necessary, depending only on $M$ and $N$, the above expression can be bounded by $a^{N}$.

A cruder first moment argument is used to handle the critical case $d=\alpha$.

Theorem 3.8. Assume $d \geqslant \alpha$. If $M \in \mathbf{N}, M \geqslant m\left(\mathbf{R}^{d}\right), \exists c^{(6)}=c^{(6)}(M)$ such that

$$
\left.P\left(N_{t}\left(S\left(\underline{t}, a, K \phi_{d, \alpha}(a)\right)\right) \geqslant \lambda\right) \leqslant \lambda^{-1} c^{(6)} \exp \left\{-\left(c^{(3)} / 2\right) K \log \log 1 / a\right)\right\},
$$

where $\lambda, K>0, \underline{t} \in T \cap\left[M^{-1}, \infty\right), a^{\alpha} \in\left(2 \mu^{-1}, \underline{t}^{-1} \wedge 3^{-\alpha}\right)$. 
Proof. Fix $\lambda, K, \underline{t}, a$ as above. Write $N_{t}\left(B\left(N_{t}^{\beta}, a\right)\right)=Z^{\beta}\left(a, \underline{t}-\mu^{-1}\right)+W_{\infty}^{\beta}(a)$ and proceed as in the proof of Theorem 3.7 to see that

$$
\begin{aligned}
& P\left(N_{t}\left(S\left(\underline{t}, a, K \phi_{d, \alpha}(a)\right)\right) \geqslant \lambda\right) \\
& \begin{aligned}
\leqslant\left(\mu ^ { - 1 } \sum _ { \beta \sim t } I \left(Z^{\beta}\left(a, \underline{t}-\mu^{-1}\right) \geqslant\right.\right. & \left.(K / 2) \phi_{d, \alpha}(a)\right) \\
& \left.+I\left(W_{\infty}^{\beta}(a) \geqslant(K / 2) \phi_{d, \alpha}(a)\right) \geqslant \lambda\right)
\end{aligned} \\
& \leqslant \lambda^{-1}\left[\mu^{-1} \sum_{\beta \sim t} P\left(Z^{\beta}\left(a, \underline{t}-\mu^{-1}\right) \geqslant(K / 2) \phi_{d, \alpha}(a)\right)\right. \\
& \left.+P\left(W_{\infty}^{\beta}(a) \geqslant(K / 2) \phi_{d, \alpha}(a)\right)\right] \\
& \leqslant \lambda^{-1}\left[e \cdot \exp \left\{-c^{(3)}(K / 2) \log \log 1 / a\right\} m\left(\mathbf{R}^{d}\right)\right. \\
& \left.\quad+\mu^{-1} \sum_{\beta \sim t} P\left(W_{\infty}^{\beta}(a) \geqslant(K / 2) \phi_{d, \alpha}(a)\right)\right] \quad \text { (by Lemma 3.2(b)). }
\end{aligned}
$$

Recall $m=\mu^{-1} \sum_{i=1}^{K_{0}} \delta_{x_{i}}$ and for $1 \leqslant i \leqslant K_{0}$, define $\omega_{i}^{1} \in \Omega^{1}$ by

$$
\omega_{i}^{1}(j)= \begin{cases}\Delta, & j \neq i \\ x_{i}, & j=i,\end{cases}
$$

and $G_{i}: \Omega \times \mathbf{R}^{d} \rightarrow \mathbf{R}$ by

$$
G_{i}(\omega, x)=\mu^{-1} \sum_{\gamma \sim t, \gamma_{0}=i} I\left(N_{t}^{\gamma} \in B(x, a)\right)=N_{t}\left(\omega_{i}^{1}, \Pi^{2} \omega\right)(B(x, a)) .
$$

If $S_{i}=\left\{\gamma \sim \underline{t}: \gamma_{0}=i\right\}$ and $\beta \in I$, then $G_{i}$ is $\mathscr{F}\left(S_{i}\right) \times \mathscr{B}\left(\mathbf{R}^{d}\right)$-measurable and $\left\{\mathscr{F}\left(S_{i}\right): i \neq \beta_{0}, i \leqslant K_{0}\right\}$ are mutually independent $\sigma$-fields whose join is independent of $\mathscr{F}(\beta)$. Therefore $\left\{G_{i}\left(\omega, N_{t}^{\beta}\right): i \neq \beta_{0}\right\}$ are conditionally independent given $\mathscr{F}(\beta)$. If $\theta=\left(2 c^{(2)}\right)^{-1} \psi_{d, \alpha}(a)^{-1}$, then on $\left\{N_{t}^{\beta} \neq \Delta\right\}$,

$$
\begin{aligned}
P\left(W_{\infty}^{\beta}(a)\right. & \left.\geqslant(K / 2) \phi_{d, \alpha}(a) \mid \mathscr{F}(\beta)\right)(\omega) \\
& =P\left(\sum_{i=1, i \neq \beta_{0}}^{K_{0}} G_{i}\left(\cdot, N_{i}^{\beta}\right) \geqslant(K / 2) \phi_{d, \alpha}(a) \mid \mathscr{F}(\beta)\right)(\omega) \\
& \leqslant \exp \left\{-\theta K \phi_{d, \alpha}(a) / 2\right\} \prod_{i=1, i \neq \beta_{0}}^{K_{0}} E^{\omega_{i}^{1}}\left(e^{\theta N_{t}\left(B\left(N_{l}^{\beta}(\omega), a\right)\right)}\right)
\end{aligned}
$$

(by the conditional independence)

$$
\begin{aligned}
\leqslant & \exp \left\{-\left(4 c^{(2)}\right)^{-1} K \log \log 1 / a\right\} \\
& \times \exp \left\{\sum_{i=1}^{K_{0}} \mu^{-1}\left(c^{(2)}\right)^{-1} \psi_{d, \alpha}(a)^{-1} P_{0}^{x_{i}}\left(\left|Y_{t}-N_{t}^{\beta}(\omega)\right| \leqslant a\right)\right\}
\end{aligned}
$$

(Proposition 2.6, Lemma 2.7) 


$$
\begin{array}{lr}
\leqslant \exp \left\{-\left(4 c^{(2)}\right)^{-1} K \log \log 1 / a+c^{(2)^{-1}} m\left(\mathbf{R}^{d}\right) a^{-\alpha} c_{1} a^{d} \underline{t}^{-d / \alpha}\right\} \\
\quad(\text { by }(2.4) \text { and } a<1 / 3) \\
\leqslant c_{1}(M) \exp \left\{-\left(4 c^{(2)}\right)^{-1} K \log \log 1 / a\right\} & \left(m\left(\mathbf{R}^{d}\right) \leqslant M, \underline{t}^{-1} \leqslant M\right) .
\end{array}
$$

Combining the above with (3.14), one gets

$$
\begin{aligned}
& P\left(N_{t}\left(S\left(\underline{t}, a, K \phi_{d, \alpha}(a)\right)\right) \geqslant \lambda\right) \\
& \leqslant \lambda^{-1}\left[e M \exp \left\{-\left(c^{(3)} / 2\right) K \log \log 1 / a\right\}\right. \\
& \left.\quad+c_{1}(M) \exp \left\{-\left(c^{(3)} / 2\right) K \log \log 1 / a\right\}\left(\mu^{-1} \sum_{\beta \sim \underline{t}} P\left(N_{t}^{\beta} \neq \Delta\right)\right)\right] \\
& \leqslant \lambda^{-1} c^{(6)}(M) \exp \left\{-\left(c^{(3)} / 2\right) K \log \log 1 / a\right\} .
\end{aligned}
$$

4. Upper bound on $X_{t}$-interpolation arguments and proof. Having obtained a probabilistic bound on $N_{t}\left(S\left(\underline{t}, a, K \phi_{d, \alpha}(a)\right)\right)$ for $\underline{t}$ fixed $\left(S\left(\underline{t}, a, K \phi_{d, \alpha}(a)\right)\right.$ as in (3.13)), we now will bound $\sup \left\{N_{t}\left(S\left(\underline{t}, a, K \phi_{d, \alpha}(a)\right)\right): 1 / M \leqslant \underline{t} \leqslant M\right\}$. This will be done by first bounding $N_{t}\left(S\left(\underline{t}, a, K \phi_{d, \alpha}(a)\right)\right)$ for $\underline{t}$ in a grid $\{j \Delta: j \in \mathbf{N}\}$ and then interpolating between grid points. The key idea (expressed more precisely in Lemma 4.3 below) is the following: If $N_{t}\left(S\left(\underline{t}, a, K \phi_{d, \alpha}(a)\right)\right)$ is small for $\underline{t}=(j+1) \Delta$ but large for some $\underline{t}_{0} \in[j \Delta,(j+1) \Delta]$, then an appreciable number of particles must have either died or travelled a distance $O(a)$ in the interval $\left[\underline{t}_{0},(j+1) \Delta\right]$. The first task is to bound these latter two probabilities in Lemmas 4.1 and 4.2 below. Clearly we must take $\Delta=o\left(a^{\alpha}\right)$ if the last probability is to be small.

$\mu \in \mathbf{N}$ will be fixed until Theorem 4.5 but it will be convenient to let the initial measure $N_{0}=m \in M_{F}^{\mu}$ vary.

Notation. If $\lambda, \Delta_{0}>0$ and $\delta_{1} \in(0,1)$, let $\underline{\Delta}_{0}=\left[\mu \Delta_{0}\right] / \mu$, and

$$
\begin{aligned}
\eta_{1}= & \eta_{1}\left(\lambda, \Delta_{0}, \delta_{1}, \mu\right) \\
= & \exp \left\{\left(2 / \Delta_{0}\right)\left(\left(1-\delta_{1}\right)^{1 / 2}-\left(1-\delta_{1}\right)\right)\right\} \\
& \times\left|\left(E^{\delta_{0}}\left(e^{-\theta N_{\Delta_{0}}\left(\mathbf{R}^{d}\right)}\right)\right)^{\lambda}-\left(E_{Q^{\delta_{0}}}\left(e^{-\theta X_{\Delta_{0}}\left(\mathbf{R}^{d}\right)}\right)\right)^{\lambda}\right|,
\end{aligned}
$$

where $\theta=2 / \Delta_{0}\left(\left(1-\delta_{1}\right)^{-1 / 2}-1\right)$.

LEMMA 4.1. (a) If $\lambda, \Delta_{0}>0$ and $\delta_{1} \in(0,1)$ then

$$
\lim _{\mu \rightarrow \infty} \eta_{1}\left(\lambda, \Delta_{0}, \delta_{1}, \mu\right)=0
$$

and

$$
P^{m}\left(\inf _{\underline{t} \leqslant \Delta_{0}} \mu^{-1} \sum_{\beta \sim \underline{t}} I\left(N_{0}^{\beta} \in S, N_{\underline{t}}^{\beta} \neq \Delta\right) \leqslant\left(1-\delta_{1}\right) m(S)\right) \leqslant \exp \left\{-\delta_{1}^{2} \lambda / 2 \Delta_{0}\right\}+\eta_{1}
$$

whenever $m \in M_{F}^{\mu}$ and $S \in \mathscr{B}\left(\mathbf{R}^{d}\right)$ satisfies $m(S) \geqslant \lambda$.

(b) If $\lambda>0, \underline{\Delta}_{0} \in T, m \in M_{F}^{\mu}$ and $S \in \mathscr{B}\left(\mathbf{R}^{d}\right)$, then

$$
P^{m}\left(\sup _{\underline{t} \leqslant \underline{\Delta}_{0}} \mu^{-1} \sum_{\beta \sim \underline{t}} I\left(N_{0}^{\beta} \in S, N_{t}^{\beta} \neq \Delta\right) \geqslant \lambda\right) \leqslant \exp \left\{-\left(\underline{\Delta}_{0}+\mu^{-1}\right)^{-1}(\lambda / 2-m(S))\right\} \text {. }
$$


Proof. (a) Fix $\lambda, \Delta_{0}>0$, and $\delta_{1} \in(0,1)$. By replacing $m$ with $m(S \cap \cdot)$ we may assume without loss of generality that $S=\mathbf{R}^{d}$. Choose $m \in M_{F}^{\mu}$ such that $m\left(\mathbf{R}^{d}\right) \geqslant \lambda$. $\left\{N_{j / \mu}\left(\mathbf{R}^{d}\right): j=0,1, \ldots\right\}$ is a martingale. The maximal inequality for submartingales therefore implies that for $\theta \geqslant 0$,

$$
\begin{aligned}
P^{m}\left(\inf _{t \leqslant \Delta_{0}} N_{t}\left(\mathbf{R}^{d}\right) \leqslant\left(1-\delta_{1}\right) m\left(\mathbf{R}^{d}\right)\right) & =P^{m}\left(\sup _{t \leqslant \Delta_{0}} e^{-\theta N_{t}\left(\mathbf{R}^{d}\right)} \geqslant e^{-\theta\left(1-\delta_{1}\right) m\left(\mathbf{R}^{d}\right)}\right) \\
\leqslant & {\left[\exp \left\{\theta\left(1-\delta_{1}\right) m\left(\mathbf{R}^{d}\right)\right\} E^{m}\left(e^{-\theta N_{\Delta_{0}}\left(\mathbf{R}^{d}\right)}\right)\right] \wedge 1 . }
\end{aligned}
$$

Divide $N_{\underline{\Delta}_{0}}\left(\mathbf{R}^{d}\right)$ into $\mu m\left(\mathbf{R}^{d}\right)$ i.i.d. contributions (corresponding to the descendants of each initial particle) to see that the above equals

$$
\begin{aligned}
{\left[\exp \left\{\theta\left(1-\delta_{1}\right)\right\} E^{\mu^{-1} \delta_{0}}\left(e^{-\theta N_{\Delta_{0}}\left(\mathbf{R}^{d}\right)}\right)^{\mu}\right]^{m\left(\mathbf{R}^{d}\right)} } & \wedge 1 \\
& \leqslant \exp \left\{\theta \lambda\left(1-\delta_{1}\right)\right\} E^{\delta_{0}}\left(e^{-\theta N_{\Delta_{0}}\left(\mathbf{R}^{d}\right)}\right)^{\lambda} .
\end{aligned}
$$

Take $\theta=\left(2 / \Delta_{0}\right)\left(\left(1-\delta_{1}\right)^{-1 / 2}-1\right)$ to get

$$
P^{m}\left(\inf _{\underline{t} \leqslant \Delta_{0}} N_{t}\left(\mathbf{R}^{d}\right) \leqslant\left(1-\delta_{1}\right) m\left(\mathbf{R}^{d}\right)\right) \leqslant \eta_{1}+\exp \left\{\theta \lambda\left(1-\delta_{1}\right)\right\} E_{Q}^{\delta_{0}}\left(e^{-\theta X_{\Delta_{0}}\left(\mathbf{R}^{d}\right)}\right)^{\lambda} .
$$

Theorem 1.2 and Knight [1981, p. 100] imply that

$$
E_{Q}^{\delta_{0}}\left(e^{-\theta X_{\Delta_{0}}\left(\mathbf{R}^{d}\right)}\right)=\exp \left\{-2 \theta\left(2+\theta \Delta_{0}\right)^{-1}\right\} .
$$

Substitute this into the previous expression to see that it is bounded by

$$
\eta_{1}+\exp \left\{-\delta_{1}^{2} \lambda / 2 \Delta_{0}\right\} \text {. }
$$

$\lim _{\mu \rightarrow \infty} \eta_{1}=0$ is clear from Theorem 2.8, and the proof of (a) is complete.

(b) The proof of (b) is similar but simpler. Use Proposition 2.6(c) in place of the above Laplace transform for $X_{\Delta_{0}}\left(\mathbf{R}^{d}\right)$.

Notation. If $M>0$, let $T_{1}(M)=\min \left\{\underline{t} \in T: N_{t}\left(\mathbf{R}^{d}\right)>M\right\}(\min \varnothing=\infty)$.

Next consider a bound on the number of particles that travel a (relatively) large distance in a short time. A simple first moment argument suffices for the present purposes ((a) below). The stronger estimate (c) will be used in the proof of the lower bound on $X_{t}$.

LemMA 4.2. Let $\underline{t} \in T^{>0}, \lambda>0, m \in M_{F}^{\mu}, M>0, \Delta_{0}>0$ and $a>0$.

(a)

$$
P^{m}\left(\mu^{-1} \sum_{\beta \sim t} I\left(\left|N_{t}^{\beta}-N_{0}^{\beta}\right|>a\right) \geqslant \lambda\right) \leqslant \lambda^{-1} m\left(\mathbf{R}^{d}\right) P_{0}^{0}(|Y(\underline{t})|>a),
$$

(b)

$$
\begin{aligned}
P^{m}\left(\mu^{-1} \sum_{\beta \sim \underline{t}} I\left(\left|N_{\underline{t}}^{\beta}-N_{0}^{\beta}\right|>a\right)>\lambda+m\left(\mathbf{R}^{d}\right) P_{0}^{0}(|Y(\underline{t})|>a)\right) \\
\leqslant \lambda^{-2} m\left(\mathbf{R}^{d}\right)\left(\underline{t}+\mu^{-1}\right) P_{0}^{0}(|Y(\underline{t})|>a),
\end{aligned}
$$


(c) $\exists c^{(7)}$ and $\mu_{1}=\mu_{1}\left(\lambda, \Delta_{0}\right) \in \mathbf{N}$ such that

$$
\begin{array}{r}
P^{m}\left(\sup _{0 \leqslant s<\Delta_{0} \wedge T_{1}(M)} \mu^{-1} \sum_{\beta \sim \underline{s}} I\left(\left|N_{\underline{s}}^{\beta}-N_{0}^{\beta}\right|>a\right)>\lambda\right) \\
\leqslant c^{(7)} \lambda^{-2} M \Delta_{0} P_{0}^{0}\left(\left|Y\left(\Delta_{0}\right)\right|>a / 2\right)
\end{array}
$$

whenever $\mu \geqslant \mu_{1}, \Delta_{0} \leqslant \lambda$ and $\lambda>8 M P_{0}^{0}\left(\left|Y\left(\Delta_{0}\right)\right|>a / 2\right)$.

Proof. (a)

$$
\begin{aligned}
E^{m}\left(\mu^{-1} \sum_{\beta \sim t} I\left(\left|N_{t}^{\beta}-N_{0}^{\beta}\right|>a\right)\right) \\
=\mu^{-1} \sum_{\beta \sim \underline{t}} P^{m}\left(\left|N_{t}^{\beta}-N_{0}^{\beta}\right|>a \mid N_{t}^{\beta} \neq \Delta\right) P^{m}\left(N_{t}^{\beta} \neq \Delta\right) \\
=P_{0}^{0}(|Y(\underline{t})|>a) m\left(\mathbf{R}^{d}\right) \quad(\text { Lemma 2.1(c), Proposition 2.2). }
\end{aligned}
$$

(a) is immediate.

(b) If $m=\mu^{-1} \sum_{i=1}^{K_{0}} \delta_{x_{i}}$, let

$$
R_{i}=\mu^{-1} \sum_{\beta \sim t, \beta_{0}=i} I\left(\left|N_{t}^{\beta}-N_{0}^{\beta}\right|>a\right), \quad 1 \leqslant i \leqslant K_{0} .
$$

Under $P^{m},\left\{R_{i}: i \leqslant K_{0}\right\}$ are i.i.d. r.v.'s such that

$$
P^{m}\left(R_{i} \in \cdot\right)=P^{\mu^{-1} \delta_{0}}\left(N_{t}\left(B(0, a)^{c}\right) \in \cdot\right)
$$

and (by (a))

$$
E\left(\sum_{i=1}^{K_{0}} R_{i}\right)=P_{0}^{0}(|Y(\underline{t})|>a) m\left(\mathbf{R}^{d}\right)
$$

Therefore

$$
\begin{aligned}
& P^{m}\left(\mu^{-1} \sum_{\beta \sim \underline{t}} I\left(\left|N_{\underline{t}}^{\beta}-N_{0}^{\beta}\right|>a\right)>\lambda+m\left(\mathbf{R}^{d}\right) P_{0}^{0}(|Y(\underline{t})|>a)\right) \\
& =P^{m}\left(\sum_{i=1}^{K_{0}} R_{i}-E^{m}\left(R_{i}\right)>\lambda\right) \\
& \leqslant \lambda^{-2} K_{0} E^{m}\left(R_{1}^{2}\right) \quad \text { (Chebychev) } \\
& =\lambda^{-2} m\left(\mathbf{R}^{d}\right) E^{\mu^{-1} \delta_{0}}\left(\mu N_{t}\left(B(0, a)^{c}\right)^{2}\right) \\
& \leqslant \lambda^{-2} m\left(\mathbf{R}^{d}\right) E_{0}^{0}\left(\int_{\left[-\mu^{-1}, \underline{t}\right)} S_{\underline{u}^{+}} I_{B(0, a)^{c}}\left(Y_{\underline{t}-\underline{u}^{+}}\right) d \lambda(\underline{u})\right) \quad \text { (Proposition 2.6(a)(ii)) } \\
& =\lambda^{-2} m\left(\mathbf{R}^{d}\right) P_{0}^{0}\left(\left|Y_{\underline{t}}\right|>a\right)\left(\underline{t}+\mu^{-1}\right) \quad \text { (by the semigroup property). }
\end{aligned}
$$


(c) Assume without loss of generality that $m\left(\mathbf{R}^{d}\right) \leqslant M$ because otherwise the above probability is zero. Assume $\mu^{-1}<\Delta_{0} \leqslant \lambda$ and

$$
\lambda>8 M P_{0}^{0}\left(\left|Y\left(\Delta_{0}\right)\right|>a / 2\right),
$$

and set $\underline{\Delta}=\left[\mu \Delta_{0}\right] / \mu>0$. Define

$$
U=\min \left\{\underline{s} \in T: \mu^{-1} \sum_{\beta \sim \underline{s}} I\left(\left|N_{\underline{s}}^{\beta}-N_{0}^{\beta}\right|>a, N_{\underline{s}}^{\beta} \neq \Delta\right)>\lambda\right\} \quad(>0)
$$

and on $\{U<\infty\}$, let

$$
S(\omega)=\left\{N_{U}^{\beta}:\left|N_{U}^{\beta}-N_{0}^{\beta}\right|>a, N_{U}^{\beta} \neq \Delta, \beta \sim U\right\} .
$$

Clearly $(x, \omega) \rightarrow I(U(\omega)<\infty, x \in S(\omega))$ is $\mathscr{B}\left(\mathbb{R}^{d}\right) \times \mathscr{A}_{U}$-measurable. Elementary reasoning gives us

$$
\begin{aligned}
& P^{m}\left(U<\Delta_{0} \wedge T_{1}(M)\right) \\
& \leqslant P^{m}\left(U<\Delta_{0} \wedge T_{1}(M), \mu^{-1} \sum_{\beta \sim \underline{\Delta}} I\left(\left|N_{\underline{\beta}}^{\beta}-N_{U}^{\beta}\right|>a / 2, N_{\underline{\beta}}^{\beta} \neq \Delta\right)>\lambda / 4\right) \\
& +P^{m}\left(U<\Delta_{0} \wedge T_{1}(M), \mu^{-1} \sum_{\beta \sim \underline{\Delta}} I\left(N_{U}^{\beta} \in S, N_{\underline{\beta}}^{\beta} \neq \Delta\right)<\lambda / 2\right) \\
& +P^{m}\left(U<\Delta_{0} \wedge T_{1}(M), \mu^{-1} \sum_{\beta \sim \Delta} I\left(\left|N_{\underline{\Delta}}^{\beta}-N_{0}^{\beta}\right|>a / 2\right)>\lambda / 4\right) \\
& =E^{m}\left(I\left(U<\Delta_{0} \wedge T_{1}(M)\right)\right. \\
& \left.\times P^{N_{U}(\omega)}\left(\mu^{-1} \sum_{\beta \sim \underline{\Delta-U(\omega)}} I\left(\left|N_{\underline{\beta}}^{\beta}-U(\omega)-N_{0}^{\beta}\right|>a / 2, N_{\underline{\beta}}^{\beta}-U(\omega) \neq \Delta\right)>\lambda / 4\right)\right) \\
& +E^{m}\left(I\left(U<\Delta_{0} \wedge T_{1}(M)\right) P^{N_{U}(\omega)}\right. \\
& \times\left(\mu^{-1} \sum_{\beta \sim \underline{\Delta-U(\omega)}} I\left(N_{0}^{\beta} \in S(\omega), N_{\underline{\beta}-U(\omega)}^{\beta} \neq \Delta\right)\right. \\
& \left.\left.<N_{0}(S(\omega)) / 2\right)\right) \\
& +64 \lambda^{-2} m\left(\mathbf{R}^{d}\right)\left(\underline{\Delta}+\mu^{-1}\right) P_{0}^{0}(|Y(\underline{\Delta})|>a / 2)
\end{aligned}
$$

(by the Strong Markov Property, (b), and (4.2) - see (4.3) below)

$$
\begin{aligned}
\leqslant & 64 \lambda^{-2} \underline{\Delta} P_{0}^{0}\left(\left|Y_{\underline{\Delta}}\right|>a / 2\right) E^{m}\left(I\left(U<\Delta_{0} \vee T_{1}(M)\right) N_{U}\left(\mathbf{R}^{d}\right)\right) \\
& +P^{m}\left(U<\Delta_{0} \wedge T_{1}(M)\right)\left(\exp \{-\lambda / 8 \underline{\Delta}\}+\eta_{1}\left(\lambda, \Delta_{0}, 1 / 2, \mu\right)\right) \\
& +128 \lambda^{-2} M \Delta_{0} P_{0}^{0}\left(\left|Y\left(\Delta_{0}\right)\right|>a / 2\right) .
\end{aligned}
$$


In the last line we have again used (b) with $m=N_{U}$ and $\Delta-U(\omega) \leqslant \Delta-\mu^{-1}$ in place of $\underline{t}$. Note that $U<T_{1}(M)$ implies (by (4.2))

$$
\lambda / 4>\lambda / 8+M P_{0}^{0}\left(\left|Y\left(\Delta_{0}\right)\right|>a / 2\right) \geqslant \lambda / 8+N_{U}\left(\mathbf{R}^{d}\right) P_{0}^{0}(|Y(\underline{\Delta})|>a / 2)
$$

and therefore we may apply (b) with $\lambda / 8$ in place of $\lambda$. We have also used Lemma 4.1(a). This is possible because $N_{U}(S)>\lambda$ on $\{U<\infty\}$. Use the above and the fact that $\underline{\Delta} \leqslant \lambda$ to get

$$
\begin{aligned}
P^{m}(U< & \left.\Delta_{0} \wedge T_{1}(M)\right) \\
& \leqslant 192 \lambda^{-2} M \Delta_{0} P_{0}^{0}\left(\left|Y\left(\Delta_{0}\right)\right|>a / 2\right)\left(1-e^{-1 / 8}-\eta_{1}\left(\lambda, \Delta_{0}, 1 / 2, \mu\right)\right)^{-1},
\end{aligned}
$$

assuming this last term is positive. Choose $\mu_{1}\left(\lambda, \Delta_{0}\right) \in \mathbf{N}$ such that $\mu_{1}>\Delta_{0}^{-1}$ and

$$
e^{-1 / 8}+\eta_{1}\left(\lambda, \Delta_{0}, 1 / 2, \mu\right) \leqslant e^{-1 / 9} \text { for } \mu \geqslant \mu_{1}\left(\lambda, \Delta_{0}\right) .
$$

Let $c^{(7)}=192(1-e-1 / 9)^{-1}$ to complete the proof.

The key idea in the interpolation argument is contained in the following result. Recall the notation (3.13).

Lemma 4.3. Let $a, K, \delta_{1}, \varepsilon, \eta>0$. Assume $\omega \in \Omega, 0 \leqslant T_{1} \leqslant T_{2}<\infty$ and $S \subset \mathbf{R}^{d}$ $\left(T_{1}, T_{2}\right.$ and $S$ may depend on $\left.\omega\right)$ satisfy

$$
\begin{gathered}
\beta \sim T_{1}, \quad N_{T_{1}}^{\beta} \in S \Rightarrow N_{T_{1}}\left(B\left(N_{T_{1}}^{\beta}, a\right) \cap S\right) \geqslant K, \\
\mu^{-1} \sum_{\beta \sim T_{2}} I\left(N_{T_{1}}^{\beta} \in S, N_{T_{2}}^{\beta} \neq \Delta\right) \geqslant\left(1-\delta_{1}\right) N_{T_{1}}(S), \\
\mu^{-1} \sum_{\beta \sim T_{2}} I\left(\left|N_{T_{2}}^{\beta}-N_{T_{1}}^{\beta}\right|>a, N_{T_{1}}^{\beta} \in S\right) \leqslant \varepsilon N_{T_{1}}(S) .
\end{gathered}
$$

Then

$$
\mu^{-1} \sum_{\beta \sim T_{2}} I\left(N_{T_{2}}^{\beta} \in S\left(T_{2}, 4 a, \eta K\right), N_{T_{1}}^{\beta} \in S\right) \geqslant\left(1-\delta_{1}-\varepsilon-\eta\right) N_{T_{1}}(S) .
$$

Proof. Fix $\omega, T_{1}, T_{2}$ and $S$ as above and let $\delta_{1}, \varepsilon, \eta \in(0,1]$. For $t \geqslant T_{1}$, let $\tilde{N}_{t}=\sum_{\beta \sim t} \delta_{N_{t}^{\beta}} I\left(N_{T_{1}}^{\beta} \in S\right)$. Also define

$$
\begin{gathered}
I_{1}=\left\{\beta \sim T_{2}:\left|N_{T_{2}}^{\beta}-N_{T_{1}}^{\beta}\right| \leqslant a\right\}, \\
A=\left\{N_{T_{2}}^{\beta}: N_{T_{2}}^{\beta} \notin S\left(T_{2}, 4 a, \eta K\right), \beta \in I_{1}\right\} .
\end{gathered}
$$

Cover $A$ by a countable collection of balls $\left\{B\left(N_{T_{2}}^{\beta_{i}}, 4 a\right): i \in J\right\}$ such that $\beta_{i} \in I_{1}$, $N_{T_{2}}^{\beta_{i}} \in A$ and $\left\{B\left(N_{T_{2}}^{\beta_{i}}, 2 a\right): i \in J\right\}$ are disjoint. Such a covering can be constructed inductively by searching for an $N_{T_{i}}^{\beta_{i}} \in A\left(\beta_{i} \in I_{1}\right)$ such that $N_{T_{2}}^{\beta_{i}} \bigcup_{j<i} B\left(N_{T_{2}}^{\beta_{j}}, 4 a\right)$ and continuing, until $A$ is covered after a finite or countable number of steps. (In 
practice $\left\{i: \omega_{i}^{1} \neq \Delta\right\}$ is finite and hence so is $J$.) Then

$$
\begin{aligned}
& \mu^{-1} \sum_{\beta \sim T_{2}} I\left(N_{T_{2}}^{\beta} \in S\left(T_{2}, 4 a, \eta K\right)^{c}, N_{T_{1}}^{\beta} \in S\right) \\
&=\mu^{-1} \sum_{\beta \sim T_{2}, \beta \in I_{1}^{c}} I\left(N_{T_{2}}^{\beta} \in S\left(T_{2}, 4 a, \eta K\right)^{c}, N_{T_{1}}^{\beta} \in S\right) \\
&+\mu^{-1} \sum_{\beta \sim T_{2}, \beta \in I_{1}} I\left(N_{T_{2}}^{\beta} \in S\left(T_{2}, 4 a, \eta K\right)^{c}, N_{T_{1}}^{\beta} \in S\right) \\
& \leqslant \varepsilon N_{T_{1}}(S)+\tilde{N}_{T_{2}}(A) \quad(\text { by }(4.6) \text { and the definition of } A) \\
& \leqslant \varepsilon N_{T_{1}}(S)+\sum_{i \in J} \tilde{N}_{T_{2}}\left(B\left(N_{T_{2}}^{\beta_{i}}, 4 a\right)\right) \\
& \leqslant \varepsilon N_{T_{1}}(S)+\sum_{i \in J} \eta N_{T_{1}}\left(B\left(N_{T_{1}}^{\beta_{i}}, a\right) \cap S\right) \quad\left(\text { by }(4.4) \text { and } N_{T_{1}}^{\beta_{i}} \in A\right) \\
& \leqslant \varepsilon N_{T_{1}}(S)+\eta \sum_{i \in J} N_{T_{1}}\left(B\left(N_{T_{2}}^{\beta_{i}}, 2 a\right) \cap S\right) \quad\left(\left|N_{T_{2}}^{\beta_{i}}-N_{T_{1}}^{\beta_{i}}\right| \leqslant a\right) \\
& \leqslant(\varepsilon+\eta) N_{T_{1}}(S) \quad(\text { the balls are disjoint }) .
\end{aligned}
$$

Subtract the above inequality from (4.5) to see that

$$
\mu^{-1} \sum_{\beta \sim T_{2}} I\left(N_{T_{2}}^{\beta} \in S\left(T_{2}, 4 a, \eta K\right), N_{T_{1}}^{\beta} \in S\right) \geqslant\left(1-\delta_{1}-\varepsilon-\eta\right) N_{T_{1}}(S) .
$$

Proposition 4.4. If $m \in M_{F}^{\mu}$ and $M \in \mathbf{N} \geqslant 2$ satisfy $m\left(\mathbf{R}^{d}\right) \leqslant M$, then $\exists c^{(8)}=c^{(8)}(M)$ such that

$$
\begin{aligned}
& P^{m}\left(\sup _{M^{-1} \leqslant \underline{t}<M} N_{\underline{t}}(S(\underline{t}, a / 2, K))>\lambda\right) \\
& \leqslant c^{(8)}\left[\exp \left(-\lambda / 32 \Delta_{0}\right)+\eta_{1}\left(\lambda, \Delta_{0}, 1 / 4, \mu\right)+\lambda^{-1} a^{-\alpha} \Delta_{0}\right. \\
& \\
& \left.\quad+\Delta_{0}^{-1} \sup _{M^{-1}<\underline{t}<M+1} P^{m}\left(N_{\underline{t}}(S(\underline{t}, 4 a, K / 4))>\lambda / 4\right)\right]
\end{aligned}
$$

whenever $a, K, \lambda>0$ and $\Delta_{0} \in T \cap[0,1]$.

Proof. Fix $a, K, \lambda$ and $\Delta_{0}$ as above and suppress dependence on these parameters in our notation. Let $t_{0} \in(0, M)$ and define $(d(x, A)$ is the distance between $x$ and the set $A$ )

$$
\begin{aligned}
T_{1} & =\inf \left\{\underline{t} \geqslant M^{-1}: N_{t}(S(\underline{t}, a / 2, K))>\lambda\right\} \quad(\inf \varnothing=\infty), \\
S(\omega) & =\left\{x: d\left(x, S\left(T_{1}, a / 2, K\right)\right)<a / 2\right\} \quad \text { on }\left\{T_{1}<\infty\right\} .
\end{aligned}
$$

If $\beta \sim T_{1}<\infty$ and $N_{T_{1}}^{\beta} \in S$ then $\left|x-N_{T_{1}}^{\beta}\right|<a / 2$ for some $x \in S\left(T_{1}, a / 2, K\right)$ and $B(x, a / 2) \subset B\left(N_{T_{1}}^{\beta}, a\right) \cap S$. Therefore

$$
N_{T_{1}}\left(B\left(N_{T_{1}}^{\beta}, a\right) \cap S\right) \geqslant N_{T_{1}}(B(x, a / 2)) \geqslant K
$$


and (4.4) holds. Lemma 4.3 (with $T_{2}=t_{0}+\Delta_{0}$ and $\varepsilon=\delta_{1}=\eta=1 / 4$ ) implies

$$
\begin{aligned}
& P^{m}\left(t_{0} \leqslant T_{1}<t_{0}+\Delta_{0}\right) \\
& \leqslant P^{m}\left(t_{0} \leqslant T_{1}<t_{0}+\Delta_{0}, \mu^{-1} \sum_{\beta \sim t_{0}+\Delta_{0}} I\left(N_{T_{1}}^{\beta} \in S, N_{t_{0}+\Delta_{0}}^{\beta} \neq \Delta\right)<(3 / 4) N_{T_{1}}(S)\right) \\
& +P^{m}\left(t_{0} \leqslant T<t_{0}+\Delta_{0}, \mu^{-1} \sum_{\beta \sim t_{0}+\Delta_{0}} I\left(N_{T_{1}}^{\beta} \in S,\left|N_{t_{0}+\Delta_{0}}^{\beta}-N_{T_{1}}^{\beta}\right|>a\right)\right. \\
& \left.\quad>(1 / 4) N_{T_{1}}(S)>\lambda / 4\right) \\
& +P^{m}\left(t_{0} \leqslant T_{1}<t_{0}+\Delta_{0}, N_{t_{0}+\Delta_{0}}\left(S\left(t_{0}+\Delta_{0}, 4 a, K / 4\right)\right) \geqslant N_{T_{1}}(S) / 4>\lambda / 4\right) .
\end{aligned}
$$

Denote the terms on the right side by I, II and III, respectively. $T_{1}$ is an $\left\{\mathscr{A}_{t}\right\}$-stopping time. An easy argument using the strong Markov property (Proposition 2.3) shows

$$
\begin{aligned}
& I=E^{m}( I\left(t_{0} \leqslant T_{1}<t_{0}+\Delta_{0}\right) \\
& \quad \times P^{N_{T_{1}}(\omega)}\left(\mu_{\beta \sim t_{0}+\Delta_{0}-T_{1}(\omega)}^{-1} \sum I\left(N_{0}^{\beta} \in S(\omega), N_{t_{0}+\Delta_{0}-T_{1}(\omega)}^{\beta} \neq \Delta\right)\right. \\
&\left.\left.<(3 / 4) N_{0}(S(\omega))\right)\right) .
\end{aligned}
$$

(Here we are implicitly using such elementary facts as $\left(\omega^{\prime}, \omega\right) \rightarrow N .\left(\omega^{\prime}\right)(S(\omega))$ is $\mathscr{A} \times \mathscr{A}_{T_{1}}$-measurable.) The definition of $T_{1}$ shows that on $\left\{T_{1}<\infty\right\}$,

$$
N_{T_{1}}(S) \geqslant N_{T_{1}}\left(S\left(T_{1}, a / 2, K\right)\right)>\lambda
$$

and therefore Lemma 4.1(a) implies

$$
I \leqslant P^{m}\left(t_{0} \leqslant T_{1}<t_{0}+\Delta_{0}\right)\left[\exp \left(-\lambda / 32 \Delta_{0}\right)+\eta_{1}\left(\lambda, \Delta_{0}, 1 / 4, \mu\right)\right] .
$$

Another application of the strong Markov property shows that

$$
\begin{aligned}
\text { II } \leqslant E^{m}( & I\left(t_{0} \leqslant T_{1}<t_{0}+\Delta_{0}\right) \\
& \left.\quad \times P^{N_{T_{1}}(\omega)}\left(\mu^{-1} \sum_{\beta \sim t_{0}+\Delta_{0}-T_{1}(\omega)} I\left(\left|N_{t_{0}+\Delta_{0}-T_{1}(\omega)}^{\beta}-N_{0}^{\beta}\right|>a\right)>\lambda / 4\right)\right), \\
\text { (4.9) II } \leqslant & E\left(I\left(t_{0} \leqslant T_{1}<t_{0}+\Delta_{0}\right) 4 \lambda^{-1} \sup _{t<M+1} N_{t}\left(\mathrm{R}^{d}\right)\right) P_{0}^{0}\left(\left|Y\left(\Delta_{0}\right)\right|>a\right)
\end{aligned}
$$


Cover $\left[M^{-1}, M\right) \cap T$ by $N$ intervals of the form $\left[t_{0}, t_{0}+\Delta_{0}\right]$ where $t_{0} \in T \cap\left[M^{-1}, M\right)$ and $N \leqslant M / \Delta_{0}+2 \leqslant 2 M / \Delta_{0}(M \geqslant 2)$. Substitute (4.8) and (4.9) into (4.7) and sum over the $N$ intervals to obtain

$$
\begin{aligned}
P^{m}\left(\sup _{M^{-1} \leqslant \underline{t}<M} N_{t}(S(\underline{t}, a / 2, K))>\lambda\right) & \\
\leqslant & \exp \left(-\lambda / 32 \Delta_{0}\right)+\eta_{1}\left(\lambda, \Delta_{0}, 1 / 4, \mu\right) \\
& +4 \lambda^{-1} P_{0}^{0}\left(\left|Y\left(\Delta_{0}\right)\right|>a\right) E^{m}\left(\sup _{\underline{t}<M+1} N_{\underline{t}}\left(\mathbf{R}^{d}\right)\right) \\
& +\left(2 M / \Delta_{0}\right) \sup _{M^{-1}<\underline{t}<M+1} P^{m}\left(N_{\underline{t}}(S(\underline{t}, 4 a, K / 4))>\lambda / 4\right) \\
& c^{(8)}(M)\left[{\exp \left(-\lambda / 32 \Delta_{0}\right)+\eta_{1}\left(\lambda, \Delta_{0}, 1 / 4, \mu\right)+\lambda^{-1} a^{-\alpha} \Delta_{0}}\left[\sup _{M^{-1}<\underline{t}<M+1} P^{m}\left(N_{t}(S(\underline{t}, 4 a, K / 4))>\lambda / 4\right)\right]\right.
\end{aligned}
$$

where we have used (2.4), Proposition 2.6(c) and the fact that $N_{t}\left(\mathbf{R}^{d}\right)$ is a martingale in the last line.

The next theorem uses nonstandard analysis to complete the proof of the upper bound on $X_{t}$. We will work in an $\omega_{1}$-saturated enlargement of a superstructure that contains our original $(\Omega, \mathscr{A})$. Recall $X_{t}$ denotes the $d$-dimensional branching diffusion of index $\alpha$ that starts at $m \in M_{F}$ under $Q^{m}$.

THEOREM 4.5. Assume $d>\alpha . \exists C<\infty$, depending only on $(\alpha, d)$, such that for any $m \in M_{F}$,

$$
X_{t}(A) \leqslant C \phi_{\alpha}-m(A) \quad \text { for all } A \in \mathscr{B}\left(\mathbf{R}^{d}\right) \text { and } t>0, Q^{m}-a . s .
$$

Proof. Take $N=4$ in Theorem 3.7 and let $K=K(4)(K(N)$ as in Theorem 3.7). Fix $m \in M_{F}$ and choose $\left\{m^{(\mu)}: \mu \in \mathbf{N}\right\} \subset M_{F}^{\mu}$ so that $m^{(\mu)} \stackrel{w}{\rightarrow} m$. Assume $M \in \mathbf{N} \geqslant 2$ satisfies $M \geqslant \sup \left\{m^{(\mu)}\left(\mathbf{R}^{d}\right): \mu \in \mathbf{N}\right\}$, and let $a_{1}=a_{1}(M+1,4)$ be as in Theorem 3.7. Define $a_{n}=e^{-n}, \Delta_{n}=a_{n}^{1+\alpha}, \lambda_{n}=8\left(\log \left(1 /\left(16 a_{n}\right)\right)\right)^{-2}(n \geqslant 4)$, fix $\mu \in{ }^{*} \mathbf{N}-\mathbf{N}$, and let $\underline{\Delta}_{n}=\left[\mu \Delta_{n}\right] / \mu$. We work on the Loeb space

$$
(* \Omega, \mathscr{F}, P)=\left(* \Omega, L(* \mathscr{A}), L\left({ }^{*} P^{m^{(\mu)}}\right)\right) .
$$

Theorem 2.8 and the nonstandard characterization of weak convergence (e.g. Anderson and Rashid [1978, Theorem 4]) imply that $N_{t} \equiv N_{t}^{(\mu)}$ is an $S$-continuous ${ }^{*} M_{F}$-valued process and $X_{t}=\mathrm{st}_{1}(N)(t) \quad\left(\mathrm{st}_{1}\right.$ is the standard part map on $\left.\mathrm{ns}\left({ }^{*} C\left([0, \infty), M_{F}\right)\right)\right)$ is the $d$-dimensional branching diffusion of index $\alpha$, that starts at $m$. This means that

$$
X_{\circ_{t}}(A)=L\left(N_{t}\right)\left(\mathrm{st}^{-1}(A)\right) \quad \forall t \in \mathrm{ns}(*[0, \infty)), A \in \mathscr{B}\left(\mathbf{R}^{d}\right) \text { a.s. }
$$


Therefore if $c_{1}=8 \times 16^{\alpha} \mathrm{K}$ and $n$ is large enough so that $16 a_{n} \leqslant a_{1}(M+1,4)$ (some easy measurability properties are left to the interested reader), one has

$$
\begin{aligned}
P\left(\sup _{M^{-1} \leqslant t \leqslant m}\right. & \left.X_{t}\left(\left\{x: X_{t}\left(B\left(x, a_{n}\right)\right) \geqslant c_{1} \phi_{\alpha}\left(a_{n}\right)\right\}\right) \geqslant \lambda_{n}\right) \\
& =P\left(\sup _{t \in\left[M^{-1}, M\right) \cap T} L\left(N_{t}\right)\left(\mathrm{st}^{-1}\left\{x: L\left(N_{t}\right)\left(\mathrm{st}^{-1}\left(B\left(x, a_{n}\right)\right)\right) \geqslant c_{1} \phi_{\alpha}\left(a_{n}\right)\right\}\right) \geqslant \lambda_{n}\right) \\
& \leqslant{ }^{\circ *} P^{m^{(\mu)}}\left(\sup _{t \in\left[M^{-1}, M\right) \cap T} N_{t}\left(S\left(t, 2 a_{n},\left(c_{1} / 2\right) \phi_{\alpha}\left(a_{n}\right)\right)\right)>\lambda_{n} / 2\right) \\
\leqslant & c^{(8)}(M)^{\circ}\left[\exp \left(-\lambda_{n} / 64 \underline{\Delta}_{n}\right)+\eta_{1}\left(\lambda_{n} / 2, \underline{\Delta}_{n}, 1 / 4, \mu\right)+2 \lambda_{n}^{-1} 4^{-\alpha} a_{n}^{-\alpha} \underline{\Delta}_{n}\right.
\end{aligned}
$$

(Proposition 4.4)

$$
\begin{aligned}
& \leqslant c_{2}(M)\left[\exp \left(-\lambda_{n} / 64 \Delta_{n}\right)+\lambda_{n}^{-1} a_{n}\right. \\
& \left.\quad+\Delta_{n}^{-1} \operatorname{osup}_{M^{-1}<t<M+1}{ }^{*} P^{m^{(\mu)}}\left(N_{t}\left(S\left(t, 16 a_{n}, K \phi_{\alpha}\left(16 a_{n}\right)\right)\right)>\left(\log \left(1 / 16 a_{n}\right)\right)^{-2}\right)\right] \\
& \leqslant c_{2}(M)\left[\exp \left(-\lambda_{n} / 64 \Delta_{n}\right)+\lambda_{n}^{-1} a_{n}+16^{4} a_{n}\right] \quad(\text { Theorem 3.7 with } N=4) .
\end{aligned}
$$

This is summable over $n$, so the Borel-Cantelli Lemma implies $\exists N(\omega)<\infty$ a.s. such that if $n \geqslant N(\omega)$, then

$$
X_{t}\left(\left\{x: X_{t}\left(B\left(x, a_{n}\right)\right) \geqslant c_{1} \phi_{\alpha}\left(a_{n}\right)\right\}\right) \leqslant \lambda_{n} \quad \forall t \in\left[M^{-1}, M\right] .
$$

Fix $\omega$ such that $N(\omega)<\infty$, let $t \in\left[M^{-1}, M\right]$ and apply Borel-Cantelli, this time with respect to the measure $X_{t}(\omega)\left(\sum \lambda_{n}<\infty\right)$, to find an $N(t, x)<\infty$ for $X_{t}$-a.s. $x$ such that

$$
X_{t}\left(B\left(x, a_{n}\right)\right) \phi_{\alpha}\left(a_{n}\right)^{-1} \leqslant c_{1} \text { for } n \geqslant N(t, x) .
$$

This shows that if $C=C(d, \alpha) \leqq e^{\alpha} c_{1}$, then

$$
\Lambda(t, \omega)=\left\{x: \limsup _{a \downarrow 0} X_{t}(B(x, a)) \phi_{\alpha}(a)^{-1} \leqslant C\right\}
$$

satisfies $X_{t}\left(\Lambda(t, \omega)^{c}\right)=0 \quad \forall t \in\left[M^{-1}, M\right]$ a.s. Apply Theorem 1.4(a) with $\nu=X_{t}$ to see that

$$
\begin{array}{rl}
X_{t}(A)=X_{t}\left(A \cap \Lambda_{t}\right) \leqslant C \phi_{\alpha}-m\left(A \cap \Lambda_{t}\right) \leqslant C \phi_{\alpha}-m(A) & \forall A \in \mathscr{B}\left(\mathbf{R}^{d}\right), \\
t & t \in\left[M^{-1}, M\right] \text { a.s. }
\end{array}
$$

Let $M \rightarrow \infty$ to complete the proof.

In the critical case $d=\alpha$ argue as above but use Theorem 3.8 in place of Theorem 3.7. Recall the functions $\phi_{\alpha}^{(i)}, i=2,3$, introduced in $\S 1$.

TheOREM 4.6. Assume $d=\alpha . \exists C<\infty$, depending only on $(\alpha, d)$, such that for any $m \in M_{F}$,

$$
X_{t}(A) \leqslant C \phi_{\alpha}^{(3)}-m(A) \quad \text { for all } A \in \mathscr{B}\left(\mathbf{R}^{d}\right) \text { and } t>0 Q^{m} \text {-a.s. }
$$


Proof. Proceed as in the proof of Theorem 4.5 up to (4.10) but with

$$
\begin{aligned}
c_{1} & =\left((80) 16^{\alpha} / c^{(3)}\right)\left(\log 1 / a_{n}\right)^{2}\left(\log \log 1 / a_{n}\right)^{-1} \\
& \equiv c_{2}\left(\log 1 / a_{n}\right)^{2}\left(\log \log 1 / a_{n}\right)^{-1} \equiv c_{2} K_{n}\left(\log 1 / a_{n}\right)
\end{aligned}
$$

to obtain (for $n \geqslant N(M)$ )

$$
\begin{aligned}
& P\left(\sup _{M^{-1} \leqslant t \leqslant M} X_{t}\left(\left\{x: X_{t}\left(B\left(x, a_{n}\right)\right) \geqslant c_{2} \phi_{\alpha}^{(3)}\left(a_{n}\right)\right\}\right) \geqslant \lambda_{n}\right) \\
& \leqslant c^{(8)}(M)\left[\exp \left(-\lambda_{n} / 64 \Delta_{n}\right)+\lambda_{n}^{-1} a_{n}\right. \\
& \left.\quad+\Delta_{n}^{-1} \quad \sup _{M^{-1}<t<M+1}{ }^{*} P^{m^{(\mu)}}\left(N_{t}\left(S\left(t, 16 a_{n},\left(10 / c^{(3)}\right) K_{n} \phi_{d, \alpha}\left(16 a_{n}\right)\right)\right)>\lambda_{n} / 8\right)\right] \\
& \leqslant c^{(8)}\left[\exp \left(-\lambda_{n} / 64 \Delta_{n}\right)+\lambda_{n}^{-1} a_{n}+\Delta_{n}^{-1} \lambda_{n}^{-1} c^{(6)} \exp \left\{-5 K_{n} \log \log 1 /\left(16 a_{n}\right)\right\}\right] \\
& \leqslant c_{2}(M)\left[\exp \left(\lambda_{n} / 64 \Delta_{n}\right)+\lambda_{n}^{-1} a_{n}+\lambda_{n}^{-1} \Delta_{n}^{-1} a_{n}^{4}\right] \quad(\text { for } n \geqslant N(M)) \\
& \leqslant c_{3}(M)\left[\exp \left(-\lambda_{n} / 64 \Delta_{n}\right)+\lambda_{n}^{-1} a_{n}\right] .
\end{aligned}
$$

The right side is summable over $n$ and the proof may be completed as in the proof of Theorem 4.5.

ThEOREM 4.7. Assume $d=\alpha . \exists C<\infty$, depending only on $(\alpha, d)$, such that for any $m \in M_{F}$,

$$
X_{t}(A) \leqslant C \phi_{\alpha}^{(2)}-m(A) \quad \text { for all } A \in \mathscr{B}\left(\mathbf{R}^{d}\right) Q^{m}-a . s . \text { for all } t>0 .
$$

Proof. Argue as in the proof of Theorem 4.5 but now for a fixed $t={ }^{\circ} \underline{t}>0$ to obtain (for $n \geqslant N(t)$ and $c_{1}>0$ ),

$$
\begin{aligned}
P\left(X_{t}(\{x:\right. & \left.\left.\left.X_{t}\left(B\left(x, a_{n}\right)\right) \geqslant c_{1} \phi_{\alpha}^{(2)}\left(a_{n}\right)\right\}\right) \geqslant \lambda_{n}\right) \\
& \leqslant{ }^{*} P^{m^{(\mu)}}\left(N_{t}\left(S\left(\underline{t}, 2 a_{n},\left(c_{1} / 2^{1+\alpha}\right) \phi_{d, \alpha}\left(2 a_{n}\right)\right) \geqslant \lambda_{n} / 2\right)\right) \\
& \leqslant 2 \lambda_{n}^{-1} c^{(6)} \exp \left\{-\left(c^{(3)} / 2\right)\left(c_{1} / 2^{1+\alpha}\right) \log \log 1 /\left(2 a_{n}\right)\right\} \quad \text { (Theorem 3.8). }
\end{aligned}
$$

Let $c_{1}=(16) 2^{\alpha} / c^{(3)}$. The above is then bounded by

$$
c_{2} n^{2} n^{-4}=c_{2} n^{-2} \text {. }
$$

This is summable and the proof proceeds as for Theorem 4.5.

5. Probability estimates for the lower bound on $X_{t}$.

Notation. If $\beta \in I, a>0, \underline{t}=|\beta| / \mu$, let

$$
\begin{aligned}
& \bar{Z}^{\beta, i}=\mu^{-1} \sum_{\gamma: \sigma(\beta ; \gamma)=i} I\left(N_{t}^{\gamma} \neq \Delta, N_{t}^{\beta} \neq \Delta\right), \quad-1 \leqslant i<|\beta|, \\
& \mathscr{F}_{2}(\beta)=\mathscr{F}(\beta) \vee \sigma\left(e^{\gamma}: \gamma \in I\right), \\
& Z^{\beta}(a)=Z^{\beta}\left(a, 2 a^{\alpha}\right)-Z^{\beta}\left(a, a^{\alpha}\right) \text { if } \underline{t}>2 a^{\alpha} .
\end{aligned}
$$

To get a lower bound on $N_{t}\left(B\left(N_{t}^{\beta}, a\right)\right)$ we will in fact find a lower bound on the smaller $Z^{\beta}(a)$. This amounts to only looking at the contribution to $N_{t} B\left(N_{t}^{\beta}, a\right)$ from those particles that branched off from $N^{\beta}$ between $\underline{t}-2 a^{\alpha}$ and $\underline{t}-a^{\alpha}$. This 
accomplishes two things. First, the scaling of $Y$ shows that these particles have a reasonable chance of ending up in $B\left(N_{t}^{\beta}, a\right)$ given that they survive. As a result their contribution should be comparable to the total number of such particles that survive (Lemma 5.1 below) and this (limiting) distribution is known from a classical result on branching processes (see (5.1), (5.2) below). Secondly, by taking a geometric sequence $a_{k} \downarrow 0$, the events $\left\{Z^{\beta}\left(a_{k}\right)\right.$ "large": $\left.k\right\}$ are conditionally independent (given $N^{\beta}$ ) and this will make it easier to find a subsequence along which $Z^{\beta}\left(a_{k_{i}}\right)$ and hence $N_{t}\left(B\left(N_{t}^{\beta}, a_{k_{i}}\right)\right)$ is large. The conditional independence will complicate things a bit.

Although $\mu \in \mathbf{N}$ and $m \in M_{F}^{\mu}$ will vary in this section, dependence on these parameters will be suppressed in our notation wherever possible. In particular we continue to write $N_{t}$ for $N_{t}^{(\mu)}$, and $P$ and $E$ for $P^{m}$ and $E^{m}$.

LEMMA 5.1. If $M>1, \beta \in I, 0<2 a^{\alpha}<\underline{t}=|\beta| / \mu$ and $i \in\left(\mu a^{\alpha}, 2 \mu a^{\alpha}\right] \cap \mathbf{N}$, then

$$
\begin{aligned}
P\left(Z^{\beta, i}(a) \geqslant\right. & \left.M^{-1} \bar{Z}^{\beta, i} \mid \mathscr{F}_{2}(\beta)\right) I\left(\left|N^{\beta}(\underline{t})-N^{\beta}(\underline{t}-i / \mu)\right| \leqslant a / 2\right) \\
& \geqslant\left(M p_{0}-1\right)(M-1)^{-1} I\left(\left|N^{\beta}(\underline{t})-N^{\beta}(\underline{t}-i / \mu)\right| \leqslant a / 2\right) \quad \text { a.s., }
\end{aligned}
$$

where $p_{0}=P_{0}^{0}(|Y(2)| \leqslant 1 / 2)$.

Proof. Fix $\beta, a, t, M$ and $i$ as above, let $N \in \mathbf{N}$ and $\left\{\gamma_{1}, \ldots, \gamma_{N}\right\}$ be a subset of $\{\gamma \in I: \quad \gamma \sim \underline{t}, \sigma(\beta ; \gamma)=i\}$. Let $A$ be the set of $\omega$ such that $N_{t}^{\beta} \neq \Delta$, $\left|N^{\beta}(\underline{t})-N^{\beta}(\underline{t}-i / \mu)\right| \leqslant a / 2$ and $\left\{\gamma_{j}: j \leqslant N\right\}=\left\{\gamma \sim \underline{t}: \sigma(\beta ; \gamma)=i, N_{\underline{t}}^{\gamma} \neq \Delta\right\}$. Then $A \in \mathscr{F}_{2}(\beta)$ and on $A$ we have

$$
\begin{aligned}
E\left(Z^{\beta, i}(a) \mid \mathscr{F}_{2}(\beta)\right)=\mu^{-1} \sum_{j=1}^{N} P\left(\left|N_{t}^{\gamma_{j}}-N_{t}^{\beta}\right| \leqslant a \mid \mathscr{F}_{2}(\beta)\right) \\
\quad=\mu^{-1} N P_{0}^{N^{\beta}(\underline{t}-i / \mu)-N_{t}^{\beta}}(|Y(i / \mu)| \leqslant a) \quad(\text { Lemma 2.5(a) and Lemma 2.1(b)) } \\
\geqslant \bar{Z}^{\beta, i} P_{0}^{0}(|Y(i / \mu)| \leqslant a / 2) \geqslant \bar{Z}^{\beta, i} p_{0} .
\end{aligned}
$$

Therefore on $\left\{N^{\beta}(\underline{t})-N^{\beta}(\underline{t}-i / \mu) \mid \leqslant a / 2\right\}$ we have

$$
E\left(Z^{\beta, i}(a) \mid \mathscr{F}_{2}(\beta)\right) \geqslant \bar{Z}^{\beta, i} p_{0} .
$$

If $q=P\left(Z^{\beta, i}(a) \geqslant M^{-1} \bar{Z}^{\beta, i} \mid \mathscr{F}_{2}(\beta)\right)$, this implies

$$
(1-q) \bar{Z}^{\beta, i} / M+q \bar{Z}^{\beta, i} \geqslant \bar{Z}^{\beta, i} p_{0} \Rightarrow q \geqslant\left(M p_{0}-1\right)(M-1)^{-1} .
$$

To get a lower bound on $\bar{Z}^{\beta, i}$, two well-known results on branching processes are needed (see Harris [1963, pp. 21-22]). Let $\left\{Z_{n}: n=0,1, \ldots\right\}$ denote a critical Galton-Watson branching process such that $Z_{0}=1$ and $P\left(Z_{1}=0\right)=P\left(Z_{1}=2\right)=$ $1 / 2$. Then

$$
\begin{gathered}
\lim _{n \rightarrow \infty} n P\left(Z_{n}>0\right)=2, \\
\lim _{n \rightarrow \infty} P\left(Z_{n} / n>z \mid Z_{n}>0\right)=e^{-2 z}, \quad z \geqslant 0 .
\end{gathered}
$$


LEMMA 5.2. $\exists c^{(9)}, c^{(10)}$, and for each $K>0$ and $a \in\left(0, e^{-1}\right) \exists \mu_{2}=\mu_{2}(a, K) \in \mathbf{N}$, such that on $\left\{N_{t}^{\beta} \neq \Delta\right\}$,

$$
\begin{aligned}
P\left(Z^{\beta}(a)>K \phi_{\alpha}(a) \mid \mathscr{F}(\beta)\right) & \\
& \geqslant c^{(9)}(\log 1 / a)^{-c^{(10)} K} \int_{\left(a^{\alpha}, 2 a^{\alpha}\right]} \underline{s}^{-1} I\left(\left|N_{t}^{\beta}-N_{\underline{t}-\underline{s}}^{\beta}\right| \leqslant a / 2\right) d \lambda(\underline{s})
\end{aligned}
$$

whenever $\beta \in I$ satisfies $\underline{t}=|\beta| / \mu>2 a^{\alpha}$ and $\mu \geqslant \mu_{2}$.

Proof. Let $a, K$ and $\beta$ satisfy the above conditions. Choose $M>1$ such that $\left(M p_{0}-1\right)(M-1)^{-1}=p_{0} / 2\left(p_{0}\right.$ as in Lemma 5.1). Define

$$
i_{0}(\omega)=\min \left\{i>\mu a^{\alpha}: \bar{Z}^{\beta, i}>0\right\} \quad(\min \varnothing=\infty)
$$

On $\left\{N_{t}^{\beta} \neq \Delta\right\}$ one has

$$
\begin{aligned}
& P\left(Z^{\beta}(a)>K \phi_{\alpha}(a) \mid \mathscr{F}(\beta)\right) \\
& \geqslant \sum_{\mu a^{\alpha}<i \leqslant 2 \mu a^{\alpha}} E\left(I\left(i_{0}=i, \bar{Z}^{\beta, i}>K M \phi_{\alpha}(a)\right)\right. \\
& \left.\quad \times P\left(Z^{\beta, i}(a)>M^{-1} \bar{Z}^{\beta, i} \mid \mathscr{F}_{2}(\beta)\right) \mid \mathscr{F}(\beta)\right) \\
& \geqslant \sum_{\mu a^{\alpha}<i \leqslant 2 \mu a^{\alpha}} I\left(N^{\beta}(\underline{t}) \neq \Delta,\left|N^{\beta}(\underline{t})-N^{\beta}(\underline{t}-i / \mu)\right| \leqslant a / 2\right)\left(p_{0} / 2\right) \\
& \quad \times P\left(\bar{Z}^{\beta, j}=0 \text { for } \mu a^{\alpha}<j<i, \bar{Z}^{\beta, i}>K M \phi_{\alpha}(a) \mid \mathscr{F}(\beta)\right) \quad(\operatorname{Lemma} 5.1) \\
& =\left(p_{0} / 2\right) \sum_{\mu a^{\alpha}<i \leqslant 2 \mu a^{\alpha}} I\left(N^{\beta}(\underline{t}) \neq \Delta,\left|N^{\beta}(\underline{t})-N^{\beta}(\underline{t}-i / \mu)\right| \leqslant a / 2\right) \\
& \quad \times \prod_{\mu a^{\alpha}<j<i} P\left(\bar{Z}^{\beta, j}=0 \mid \mathscr{F}(\beta)\right) P\left(\bar{Z}^{\beta, i}>K M \phi_{\alpha}(a) \mid \mathscr{F}(\beta)\right) .
\end{aligned}
$$

We have used the conditional independence of $\left\{\bar{Z}^{\beta, i}\right\}$ given $\mathscr{F}(\beta)$ in the last (let $a \rightarrow \infty$ in Lemma 3.1(a)). $\bar{Z}^{\beta, j}$ is also conditionally independent of $\mathscr{F}(\beta)$ given $\sigma\left(I\left(N_{t}^{\beta} \neq \Delta\right)\right)$ because it is a function of $I\left(N_{t}^{\beta} \neq \Delta\right)$ and $\left(e^{\gamma \mid k}:|\gamma|=|\beta|, k \geqslant|\beta|-\right.$ $\sigma(\beta ; \gamma))$. Therefore (5.3) implies that on $\left\{N_{t}^{\beta} \neq \Delta\right\}$,

$$
\begin{aligned}
& P\left(Z^{\beta}(a)>K \phi_{\alpha}(a) \mid \mathscr{F}(\beta)\right) \\
& \geqslant\left(p_{0} / 2\right) \sum_{\mu a^{\alpha}<i \leqslant 2 \mu a^{\alpha}} I\left(N^{\beta}(\underline{t}) \neq \Delta,\left|N^{\beta}(\underline{t})-N^{\beta}(\underline{t}-i / \mu)\right| \leqslant a / 2\right) \\
& \times \prod_{\mu a^{\alpha}<j<i} P\left(\bar{Z}^{\beta, j}=0 \mid N_{\underline{t}}^{\beta} \neq \Delta\right) P\left(\bar{Z}^{\beta, i}>K M \phi_{\alpha}(a) \mid \bar{Z}^{\beta, i}>0\right) \\
& \times P\left(\bar{Z}^{\beta, i}>0 \mid N_{t}^{\beta} \neq \Delta\right) .
\end{aligned}
$$


Each $\mu \bar{Z}^{\beta, j}$ is equal in law to $Z_{j}$ ( $j$ fixed), where $Z_{j}$ is as in (5.1), (5.2). Those results and (5.4) show $\exists \mu_{2}=\mu_{2}(a, K) \in \mathbf{N}$ such that if $\mu \geqslant \mu_{2}$, then

$$
\begin{aligned}
& P\left(Z^{\beta}(a)>K \phi_{\alpha}(a) \mid \mathscr{F}(\beta)\right) \\
& \geqslant\left(p_{0} / 2\right) \sum_{\mu a^{\alpha}<i \leqslant 2 \mu a^{\alpha}} I\left(N_{\underline{t}}^{\beta} \neq \Delta,\left|N^{\beta}(\underline{t})-N^{\beta}(\underline{t}-i / \mu)\right| \leqslant a / 2\right) \\
& \quad \times\left[\prod_{\mu a^{\alpha}<j<i}(1-3 / j)\right] i^{-1} \exp \left(-3 K M \phi_{\alpha}(a)(i / \mu)^{-1}\right) \\
& . \geqslant\left(p_{0} / 2\right) e^{-4}(\log 1 / a)^{-3 K M} \int_{\left(a^{\alpha}, 2 a^{\alpha}\right]} \underline{s}^{-1} I\left(\left|N^{\beta}(\underline{t})-N^{\beta}(\underline{t}-\underline{s})\right| \leqslant a / 2\right) d \lambda(\underline{s}) \\
& \times I\left(N_{\underline{t}}^{\beta} \neq \Delta\right) .
\end{aligned}
$$

This proves the result with $c^{(9)}=\left(p_{0} / 2\right) e^{-4}$ and $c^{(10)}=3 M$.

Notation. $\tilde{Y}(u)=Y\left(e^{u}\right) e^{-u / \alpha}, a_{j}=2^{-j / \alpha}, j_{n}=2^{n}, R_{n}=\left\{a_{j}: j_{n}<j \leqslant j_{n+1}\right\}$.

REMARK 5.3. If $\alpha=2, \tilde{Y}$ is an Ornstein-Uhlenbeck process and is stationary and ergodic if $Y(0) \equiv 0$. A simple calculation shows that in general $\left\{\tilde{Y}_{u} \mid u \in \mathbf{R}\right\}$ is a homogeneous Markov process and is stationary and ergodic if $Y(0) \equiv 0$.

LEMMA 5.4. $\exists K^{(1)}>0$ and sequences $\left\{\lambda_{n}^{0}\right\}$ and $\left\{\eta_{2}(n, \mu): n, \mu \in \mathbf{N}\right\}$ such that

$$
\lim _{n \rightarrow \infty} \dot{\lambda}_{n}^{0}=0, \quad \lim _{\mu \rightarrow \infty} \eta_{2}(n, \mu)=0 \text { for each } n,
$$

and

$$
\begin{gathered}
\mu^{-1} \sum_{\beta \sim \underline{t}} P\left(Z^{\beta}(a) \leqslant K^{(1)} \phi_{\alpha}(a) \forall a \in R_{n} \mid \mathscr{F}_{1}(\beta)\right) I\left(N_{\underline{t}}^{\beta} \neq \Delta\right) \\
\leqslant N_{\underline{t}}\left(\mathbf{R}^{d}\right)\left(\lambda_{n}^{0}+\eta_{2}(n, \mu)\right)
\end{gathered}
$$

whenever $\underline{t}>2 a_{j_{n}}^{\alpha}, \underline{t} \in T$.

Proof. Let $K^{(1)}=\left(4 c^{(10)}\right)^{-1}, n \in \mathbf{N} \geqslant 2$, define

$$
\mu(n)=\max \left\{\mu_{2}\left(a, K^{(1)}\right): a \in R_{n}\right\} \quad\left(\mu_{2}\right. \text { as in Lemma 5.2), }
$$

and assume $\mu \geqslant \mu(n)$. Finally let $\beta \in I$ satisfy $\underline{t} \equiv|\beta| / \mu>2 a_{j_{n}}^{\alpha}$ Lemma 3.1(a) shows that $\left\{Z^{\beta}(a): a \in R_{n}\right\}$ are conditionally independent given $\mathscr{F}(\beta)\left(a_{j-1}^{\alpha}=\right.$ $\left.2 a_{j}^{\alpha}\right)$. The previous lemma therefore shows that on $\left\{N_{\underline{t}}^{\beta} \neq \Delta\right\}$,

$$
\begin{aligned}
& P\left(Z^{\beta}(a) \leqslant K^{(1)} \phi_{\alpha}(a) \forall a \in R_{n} \mid \mathscr{F}(\beta)\right) \\
& \quad \leqslant \prod_{j_{n}<j \leqslant j_{n+1}}\left[1-c^{(9)}\left(\log 1 / a_{j}\right)^{-1 / 4} \int_{\left(a_{j}^{\alpha}, a_{j-1}^{\alpha}\right]} \underline{s}^{-1} I\left(\left|N_{\underline{t}}^{\beta}-N_{t-\underline{s}}^{\beta}\right| \leqslant a_{j} / 2\right) d \lambda(\underline{s})\right] \\
& \quad \leqslant \exp \left\{-c_{1} j_{n}^{-1 / 4} \int_{\left(a_{\left.j_{n+1} a_{j_{n}}^{\alpha}\right]} \underline{s}^{-1} I\left(\left|N_{t}^{\beta}-N_{t-\underline{s}}^{\beta}\right| \underline{s}^{-1 / \alpha} \leqslant 2^{-1-1 / \alpha}\right) d \lambda(\underline{s})\right\} .}\right.
\end{aligned}
$$


Integrate over $\left\{N_{\underline{t}}^{\beta} \neq \Delta\right\}$ and use Lemma 2.1(c) to see that

$$
\begin{array}{rl}
\mu^{-1} \sum_{\beta \sim t} & P\left(Z^{\beta}(a) \leqslant K^{(1)} \phi_{\alpha}(a) \forall a \in R_{n} \mid \mathscr{F}_{1}(\beta)\right) I\left(N_{\underline{t}}^{\beta} \neq \Delta\right) \\
\leqslant & {\left[\mu^{-1} \sum_{\beta \sim \underline{t}} I\left(N_{\underline{t}}^{\beta} \neq \Delta\right)\right]} \\
& \times E_{0}^{0}\left(\exp \left\{-c_{1} j_{n}^{-1 / 4} \int_{\left(a_{j_{n+1}}^{\alpha}, a_{j_{n}}^{\alpha}\right]} \underline{s}^{-1} I\left(\left|Y_{\underline{s}}\right| \underline{s}^{-1 / \alpha} \leqslant 2^{-1-1 / \alpha}\right) d \lambda(\underline{s})\right\}\right) .
\end{array}
$$

Standard weak convergence arguments show $\exists \eta=\eta(n, \mu)$ such that $\lim _{\mu \rightarrow \infty} \eta(n, \mu)$ $=0$ and $(5.5)$ is bounded by

$$
\begin{gathered}
N_{t}\left(\mathbf{R}^{d}\right)\left[E_{0}^{0}\left(\exp \left\{-c_{1} j_{n}^{-1 / 4} \int_{a_{j_{n+1}}^{\alpha}}^{a_{j_{n}}^{\alpha}} s^{-1} I\left(\left|Y_{s}\right| s^{-1 / \alpha} \leqslant 2^{-1-1 / \alpha}\right) d s\right\}\right)+\eta\right] \\
=N_{t}\left(\mathbf{R}^{d}\right)\left[\lambda_{n}^{0}+\eta\right]
\end{gathered}
$$

where

$$
\lambda_{n}^{0}=E_{0}^{0}\left(\exp \left\{-c_{1} j_{n}^{-1 / 4} \int_{0}^{j_{n} \log 2} I\left(\left|\tilde{Y}_{u}\right| \leqslant 2^{-1-1 / \alpha}\right) d u\right\}\right)
$$

The Ergodic Theorem (recall Remark 5.3) implies $\lim _{n \rightarrow \infty} \lambda_{n}^{0}=0$. To complete the proof, define

$$
\eta_{2}(n, \mu)= \begin{cases}1 & \text { if } \mu<\mu(n) \\ \eta(n, \mu) & \text { if } \mu \geqslant \mu(n)\end{cases}
$$

Notation. If $R$ is a finite subset of $(0, \infty), f: R \rightarrow(0, \infty), \nu \in M_{F}, t>0$ and $c>0$, let

$$
S_{1}(\nu, R, f)=\{x: \nu(B(x, a))<f(a) \forall a \in R\}, \quad c R=\{c a: a \in R\} .
$$

Recall the definition of $T_{1}(M)$ given before Lemma 4.2 .

THEOREM 5.5. There is a positive sequence $\left\{\lambda_{n}^{1}\right\}$ which decreases to zero, a sequence of natural numbers $\left\{\mu_{n}^{0}\right\}$, and for each $M, N \in \mathbf{N}$, an $n_{0}=n_{0}(M, N)$ such that

$$
P\left(N_{\underline{t}}\left(S_{1}\left(N_{\underline{t}}, R_{n}, K^{(1)} \phi_{\alpha}\right)\right) \geqslant M \lambda_{n}^{1}, \underline{t}<T_{1}(M)\right) \leqslant a_{j_{n+1}}^{N},
$$

whenever $n \geqslant n_{0}, \mu \geqslant \mu_{n}^{0}$ and $\underline{t} \in T \cap\left(2 a_{j_{n}}^{\alpha}, M\right]$.

Proof. Let $\lambda_{n}^{1}=2 \lambda_{n}^{0}+n^{-2}$ and choose $\mu_{n}^{0} \in \mathbf{N}$ such that

$$
\eta_{2}(n, \mu)<\lambda_{n}^{0} \quad \text { and } \quad \mu^{-1} \leqslant a_{j_{n}}^{\alpha} \quad \text { if } \mu \geqslant \mu_{n}^{0}
$$


(Here $\lambda_{n}^{0}$ and $\eta_{2}$ are as in Lemma 5.4.) Fix $n, M, N \in \mathbf{N}, \mu \in \mathbf{N}$ satisfying $\mu \geqslant \mu_{n}^{0}$, and $\underline{t} \in T \cap\left(2 a_{j_{n}}^{\alpha}, M\right]$. We may assume that $m\left(\mathbf{R}^{d}\right) \leqslant M$ because otherwise $P\left(\underline{t}<T_{1}(M)\right)=0$. Let

$$
\begin{aligned}
& W_{t}(n)=\mu^{-1} \sum_{\beta \sim t} I\left(Z^{\beta}(a) \leqslant K^{(1)} \phi_{\alpha}(a) \forall a \in R_{n}, N_{t}^{\beta} \neq \Delta\right), \\
& \bar{W}_{t}(n)=\mu^{-1} \sum_{\beta \sim t} P\left(Z^{\beta}(a) \leqslant K^{(1)} \phi_{\alpha}(a) \forall a \in R_{n}, N_{t}^{\beta} \neq \Delta \mid \mathscr{F}_{1}(\beta)\right)
\end{aligned}
$$

and note that

$$
N_{t}\left(S_{1}\left(N_{t}, R_{n}, K^{(1)} \phi_{\alpha}\right)\right) \leqslant W_{t}(n) .
$$

If $p \in \mathbf{N}$, then

$$
E\left(\left(W_{t}(n)-\bar{W}_{t}(n)\right)^{2 p}\right)=\mu^{-2 p} \sum_{\beta_{1} \sim t \cdots \beta_{2 p} \sim t} E\left(\pi\left(\beta_{1}, \ldots, \beta_{2 p}\right)\right),
$$

where

$$
\begin{aligned}
\pi\left(\beta_{1}, \ldots, \beta_{2 p}\right)=\prod_{i=1}^{2 p}\left[I\left(Z^{\beta_{i}}(a) \leqslant K^{(1)} \phi_{\alpha}(a) \forall a \in R_{n}, N_{t}^{\beta_{i}} \neq \Delta\right)\right. \\
\left.-P\left(Z^{\beta_{i}}(a) \leqslant K^{(1)} \phi_{\alpha}(a) \forall a \in R_{n}, N_{t}^{\beta_{i}} \neq \Delta \mid \mathscr{F}_{1}\left(\beta_{i}\right)\right)\right] .
\end{aligned}
$$

Fix $\beta_{1}, \ldots, \beta_{2 p} \sim \underline{t}$ and suppose $\sigma\left(\left\{\beta_{i}: i \neq i_{0}\right\} ; \beta_{i_{0}}\right)>\mu 2 a_{j_{n}}^{\alpha}$ for some $i_{0} \leqslant 2 p$. Lemma 3.1(c) implies that for $i \neq i_{0}$ and $j \geqslant j_{n}, Z^{\beta_{i}}\left(a_{j}\right)$ is $\mathscr{F}\left(\hat{S}^{\beta_{i_{0}}}\left(2 a_{j_{n}}^{\alpha}\right)\right)$-measurable and Lemma 3.1(b) implies that for $j \geqslant j_{n}, Z^{\beta_{i_{0}}}\left(a_{j}\right)$ is $\mathscr{G}\left(S^{\beta_{i_{0}}}\left(2 a_{j_{n}}^{\alpha}\right)\right) \vee \mathscr{F}_{1}\left(\beta_{i_{0}}\right)$ measurable. Therefore Lemma 3.1(d) gives us

$$
\begin{aligned}
P\left(Z^{\beta_{i_{0}}}(a) \leqslant K^{(1)} \phi_{\alpha}(a) \forall a \in R_{n}, N_{t}^{\beta_{i_{0}}} \neq \Delta \mid \mathscr{F}_{1}\left(\beta_{i_{0}}\right) \vee \mathscr{F}\left(\hat{S}^{\beta_{i_{0}}}\left(2 a_{j_{n}}^{\alpha}\right)\right)\right) \\
=P\left(Z^{\beta_{i_{0}}}(a) \leqslant K^{(1)} \phi_{\alpha}(a) \forall a \in R_{n}, N_{t}^{\beta_{i_{0}}} \neq \Delta \mid \mathscr{F}_{1}\left(\beta_{i_{0}}\right)\right) .
\end{aligned}
$$

By first conditioning with respect to $\mathscr{F}_{1}\left(\beta_{i_{0}}\right) \vee \mathscr{F}\left(\hat{S}^{\beta_{i o}}\left(2 a_{j_{n}}^{\alpha}\right)\right)$ we see from the above that $E\left(\pi\left(\beta_{1}, \ldots, \beta_{2 p}\right)\right)=0$. (5.8) now leads to

$$
\begin{aligned}
E\left(\left(W_{t}(n)-\bar{W}_{t}(n)\right)^{2 p}\right) & \leqslant \mu^{-2 p} \underset{\beta_{1}, \ldots, \beta_{2 p} \sim t}{2 a_{j_{n}}^{\alpha}} P\left(N_{t}^{\beta_{i}} \neq \Delta, i=1, \ldots, 2 p\right) \\
& \leqslant c_{1}(p, M) a_{j_{n}}^{p \alpha}
\end{aligned}
$$

(Lemma 3.3, Proposition 2.6(c) and $\underline{t} \vee m\left(\mathbf{R}^{d}\right) \leqslant M$ ). Therefore

$$
\begin{aligned}
P\left(N_{t}(\right. & \left.\left.S_{1}\left(N_{t}, R_{n}, K^{(1)} \phi_{\alpha}\right)\right) \geqslant M \lambda_{n}^{1}, \underline{t}<T_{1}(M)\right) \\
& \leqslant P\left(W_{t}(n) \geqslant M \lambda_{n}^{1}, \underline{t}<T_{1}(M)\right) \quad(\text { by }(5.7)) \\
& \leqslant P\left(W_{t}(n)-\bar{W}_{t}(n) \geqslant n^{-2}\right)+P\left(\bar{W}_{t}(n) \geqslant 2 M \lambda_{n}^{0}, \underline{t}<T_{1}(M)\right) \\
& \leqslant c_{1}(p, M) n^{4 p} a_{j_{n}}^{p \alpha}+P\left(N_{t}\left(\mathbf{R}^{d}\right)\left(\lambda_{n}^{0}+\eta_{2}(n, \mu)\right) \geqslant M\left(2 \lambda_{n}^{0}\right), \underline{t}<T_{1}(M)\right)
\end{aligned}
$$

$((5.9)$ and Lemma 5.4)

$$
=c_{1}(p, M) n^{4 p} a_{j_{n+1}}^{p \alpha / 2} \quad(\text { by }(5.6)) .
$$


Let $p \geqslant 2(N+1) / \alpha$ and then choose $n_{0}=n_{0}(M, N)$ so that the above is less than $a_{j_{n+1}}^{N}$ if $n \geqslant n_{0}$.

The previous estimate is far from optimal if $d=\alpha$. The problem is that in this case $Z^{\beta}(a)$ is of smaller order than $N_{t}\left(B\left(N_{t}^{\beta}, a\right)\right)$ as $a \downarrow 0$, as an easy first moment argument will indicate (see Lemma 5.7 and its proof). The neighborhood recurrence that occurs in the critical case leads to a significant contribution to $N_{t}\left(B\left(N_{t}^{\beta}, a\right)\right)$ from particles that branched off as far away as $t-a^{\varepsilon}$ for any $\varepsilon>0$. To get a better lower estimate we rely on a very crude method that uses only the mean and variance (see Lemma 5.6). The lower bounds obtained in this way and hence the lower bound on $X_{t}$ obtained in Theorem 6.5 are therefore clearly not optimal.

The condition $d=\alpha$ will be in force for the rest of this section.

LEMMA 5.6. Let $Z \geqslant 0$ be a random variable on a probability space $(\Omega, \mathscr{F}, Q)$ and let $\mathscr{G}$ be a sub- $\sigma$-algebra of $\mathscr{F}$. If $c(\omega)$ is $\mathscr{G}$-measurable and satisfies

$$
c E(Z \mid \mathscr{G}) \geqslant(\operatorname{Var}(Z \mid \mathscr{G}))^{1 / 2} \text { a.s., }
$$

then

$$
P(Z>E(Z \mid \mathscr{G}) / 2 \mid \mathscr{G}) \geqslant 4^{-1}(1+c)^{-2} \text { a.s. }
$$

Proof. By working with a regular conditional probability for $Z$ given $\mathscr{G}$ (Ash [1972, Theorem 6.6.5]) we may assume without loss of generality that $\mathscr{G}=\{\phi, \Omega\}$. Therefore assume $c \mu \geqslant \sigma$ where $\mu$ and $\sigma^{2}$ are the mean and variance of $Z$ and $c \in \mathbf{R}$.

$$
\begin{aligned}
(\mu+\sigma) P(Z>\mu / 2)^{1 / 2} & \geqslant\left(\int Z^{2} d Q\right)^{1 / 2} P(Z>\mu / 2)^{1 / 2} \\
& \geqslant \int Z I(Z>\mu / 2) d Q \\
& =\mu-\int Z I(Z \leqslant \mu / 2) d Q \geqslant \mu / 2 \\
& \Rightarrow P(Z>\mu / 2) \geqslant \mu^{2} /\left(4(\mu+\sigma)^{2}\right) \geqslant 4^{-1}(1+c)^{-2} .
\end{aligned}
$$

Notation. If $\beta \sim \underline{t}$ and $a^{\alpha / 2} \in(0, \underline{t} \wedge 1)$, let

$$
\begin{aligned}
& \bar{Z}^{\beta}(a)=Z^{\beta}\left(a, a^{\alpha / 2}\right)-Z^{\beta}\left(a, a^{\alpha}\right), \\
& \bar{N}_{s}^{\beta}=N_{t}^{\beta}-N_{t-s}^{\beta} \text { if } s \in[0, \underline{t}] \text { and }\left\{N_{!}^{\beta} \neq \Delta\right\}, \\
& H^{\beta}(a)=\int_{\left(a^{\alpha}, a^{\alpha / 2}\right]} \underline{s}^{-1} p\left(\bar{N}_{\underline{s}}^{\beta} / \underline{s}^{1 / \alpha}\right) d \lambda(\underline{s}) \\
& \quad \text { on }\left\{N_{t}^{\beta} \neq \Delta\right\}, \text { where } p(y) d y=P_{0}^{0}\left(Y_{1} \in d y\right) .
\end{aligned}
$$

LEMMA 5.7. There are constants $c^{(11)}, c^{(12)}$ such that if $\beta \in I, t=|\beta| / \mu$, and $a^{\alpha / 2} \in\left((2 / \mu)^{1 / 2}, \underline{t} \wedge(1 / 2)\right)$, then

$$
P\left(\bar{Z}^{(\beta)}(a)>c^{(11)} a^{\alpha} H^{\beta}(a) \mid \mathscr{F}(\beta)\right) \geqslant c^{(12)} H^{\beta}(a)(\log 1 / a)^{-1} \text { a.s. on }\left\{N_{\underline{t}}^{\beta} \neq \Delta\right\} \text {. }
$$


Proof. Let $\beta, \underline{t}, a$ be as above, and work on $\left\{N_{t}^{\beta} \neq \Delta\right\}$ throughout. An easy computation using Lemma 2.5(b) leads to

$$
E\left(\bar{Z}^{\beta}(a) \mid \mathscr{F}(\beta)\right)=\int_{\left(a^{\alpha}, a^{\alpha / 2}\right]} P_{0}^{\bar{N}_{s}^{\beta}}(|Y(\underline{s})| \leqslant a) d \lambda(\underline{s}) .
$$

Let

$$
c_{1}=\inf \left\{\left|\left\{x \in B\left(x_{0}, r\right):|x| \leqslant\left|x_{0}\right|\right\}\right| r^{-d}:\left|x_{0}\right| \geqslant r>0\right\}>0 .
$$

(Here $|A|$ is the Lebesgue measure of $A$.) Recalling that the density of $Y_{1}$ under $P_{0}^{0}$, $p(y)$, is symmetric and decreasing in $|y|$ (see (2.4)), one gets for any $x_{0} \in \mathbf{R}^{d}$ and $r \in(0,1]$

$$
P_{0}^{x_{0}}(|Y(1)| \leqslant r)=\int_{B\left(-x_{0}, r\right)} p(y) d y \geqslant c_{2} p\left(x_{0}\right) r^{d}
$$

for some $c_{2}>0$, independent of $\left(r, x_{0}\right)$. (If $\left|x_{0}\right| \geqslant r, c_{2}=c_{1}$ will work and clearly some positive constant works for $\left|x_{0}\right|<r \leqslant 1$.) A scaling argument now shows that (5.10) implies

$$
\begin{aligned}
E\left(\bar{Z}^{\beta}(a) \mid \mathscr{F}(\beta)\right) & \geqslant c_{2} a^{d} \int_{\left(a^{\alpha}, a^{\alpha / 2}\right]} p\left(\bar{N}_{\underline{s}}^{\beta} / \underline{s}^{1 / \alpha}\right) \underline{s}^{-1} d \lambda(\underline{s}) \\
& =c_{2} a^{\alpha} H^{\beta}(a) .
\end{aligned}
$$

Turning to the conditional variance, we have (by Lemma 3.1(a))

$$
\operatorname{Var}\left(\bar{Z}^{\beta}(a) \mid \mathscr{F}(\beta)\right) \leqslant \sum_{a^{\alpha}<i / \mu \leqslant a^{\alpha / 2}} E\left(Z^{\beta, i}(a)^{2} \mid \mathscr{F}(\beta)\right) .
$$

It is easy to see that

$$
P\left(Z^{\beta, i}(a) \in \cdot \mid \mathscr{F}(\beta)\right)=E^{\mu^{-1} \delta_{N_{i}} \beta_{/ \mu}}\left(N_{i / \mu}(B(0, a)) \in \cdot\right),
$$

in fact we showed the corresponding Laplace transforms were equal in the proof of Lemma 3.2(a). Proposition 2.6(a(ii)) and (5.12) therefore give

$$
\begin{aligned}
& \operatorname{Var}\left(\bar{Z}^{\beta}(a) \mid \mathscr{F}(\beta)\right) \\
& \quad \leqslant \int_{\left(a^{\alpha}, a^{\alpha / 2}\right]} E_{0^{s}}^{\bar{N}_{s}^{\beta}}\left(I_{B(0, a)}\left(Y_{\underline{s}}\right) \int_{\left[-\mu^{-1}, \underline{s}\right)} S_{\underline{u}^{+}} I_{B(0, a)}\left(Y_{\underline{s}-\underline{u}^{+}}\right) d \lambda(\underline{u})\right) d \lambda(\underline{s}) \\
& \quad \leqslant c_{3} \int_{\left(a^{\alpha}, a^{\alpha / 2}\right]} E_{0}^{\bar{N}_{s}^{\beta}}\left(I_{B(0, a)}\left(Y_{\underline{s}}\right)\right) \int_{\left[-\mu^{-1}, \underline{s}\right)}\left(a^{\alpha} / \underline{u}^{+}\right) \wedge 1 d \lambda(\underline{u}) d \lambda(\underline{s}) \\
& \quad \leqslant c_{3} E\left(\bar{Z}^{\beta}(a) \mid \mathscr{F}(\beta)\right)\left[a^{\alpha}+2 / \mu+a^{\alpha} \log \left(a^{\alpha / 2} /\left(a^{\alpha}-\mu^{-1}\right)\right)\right] \quad(\text { by }( \\
& \quad \leqslant c_{4}\left(a^{\alpha} \log 1 / a\right) E\left(\bar{Z}^{\beta}(a) \mid \mathscr{F}(\beta)\right) \quad\left(\text { by the bounds on } a^{\alpha}\right) .
\end{aligned}
$$

Use (5.11) to get an upper bound on $a^{\alpha}$ and substitute into the above to get

$$
\operatorname{Var}\left(\bar{Z}^{\beta}(a) \mid \mathscr{F}(\beta)\right) \leqslant c_{5}\left((\log 1 / a) / H^{\beta}(a)\right) E\left(\bar{Z}^{\beta}(a) \mid \mathscr{F}(\beta)\right)^{2} .
$$


Lemma 5.6 and (5.11) imply

$$
\begin{gathered}
P\left(\bar{Z}^{\beta}(a) \geqslant\left(c_{2} / 2\right) a^{\alpha} H^{\beta}(a) \mid \mathscr{F}(\beta)\right) \\
\geqslant 4^{-1}\left(1+c_{5}^{1 / 2}\left((\log 1 / a) / H^{\beta}(a)\right)^{1 / 2}\right)^{-2} \text { a.s. } \\
\geqslant c^{(12)} H^{\beta}(a)(\log 1 / a)^{-1},
\end{gathered}
$$

the last because $H^{\beta}(a) /(\log 1 / a)$ is uniformly bounded above.

Notation. Let $b_{j}=e^{-2^{j}}$ and $\bar{R}_{n}=\left\{b_{j}: j_{n}<j \leqslant j_{n+1}\right\}$.

The fact that $b_{j+1}^{1 / 2}=b_{j}$ and Lemma 3.1(a) show that

(5.13) $\left\{\bar{Z}^{\beta}\left(b_{j}\right): b_{j}^{\alpha / 2}<|\beta| / \mu\right\}$ are conditionally independent given $\mathscr{F}(\beta)$.

LEMMA 5.8. $\exists K^{(2)}>0$ and sequences $\left\{\lambda_{n}^{(2)}: n \in \mathbf{N}\right\},\left\{\eta_{3}(n, \mu): n, \mu \in \mathbf{N}\right\}$ such that

$$
\lim _{n \rightarrow \infty} \lambda_{n}^{(2)}=0, \quad \lim _{\mu \rightarrow \infty} \eta_{3}(n, \mu)=0 \text { for each } n,
$$

and

$$
\begin{aligned}
\mu^{-1} \sum_{\beta \sim \underline{t}} P\left(\bar{Z}^{\beta}(b)\right. & \left.\leqslant K^{(2)} \phi_{\alpha}^{(1)}(b) \forall b \in \bar{R}_{n} \mid \mathscr{F}_{1}(\beta)\right) I\left(N_{\underline{t}}^{\beta} \neq \Delta\right) \\
& \leqslant N_{\underline{t}}\left(\mathbf{R}^{d}\right)\left(\lambda_{n}^{(2)}+\eta_{3}(n, \mu)\right)
\end{aligned}
$$

whenever $\underline{t}>b_{j_{n}}^{\alpha / 2}, \underline{t} \in T$.

Proof. Let $\mu, n \in \mathbf{N}$ satisfy

$$
(2 / \mu)^{1 / 2}<b_{j_{n+1}}^{\alpha / 2}<b_{j_{n}}^{\alpha / 2}<1 / 2,
$$

and let $\underline{t}>b_{j_{n}}^{\alpha / 2}$. If $c>0$ and $\beta \sim \underline{t}$, then (5.13) and the previous lemma show that on $\left\{N_{\underline{t}}^{\beta} \neq \Delta\right\}$,

$$
\begin{aligned}
P\left(\bar{Z}^{\beta}(b) \leqslant c \phi_{\alpha}^{(1)}(b) \forall b \in \bar{R}_{n} \mid \mathscr{F}(\beta)\right) & \\
\leqslant & \prod_{j_{n}<j \leqslant j_{n+1}}\left[1-I\left(H^{\beta}\left(b_{j}\right) \geqslant\left(c / c^{(11)}\right) \log 1 / b_{j}\right)\right. \\
& \left.\times P\left(\bar{Z}^{\beta}\left(b_{j}\right)>c^{(11)} b_{j}^{\alpha} H^{\beta}\left(b_{j}\right) \mid \mathscr{F}(\beta)\right)\right] \\
& \leqslant \exp \left\{-\sum_{j=j_{n}+1}^{j_{n+1}} I\left(H^{\beta}\left(b_{j}\right) \geqslant\left(c / c^{(11)}\right) 2^{j}\right) c^{(12)} H^{\beta}\left(b_{j}\right) 2^{-j}\right\} .
\end{aligned}
$$

Integrate the above over $\left\{N_{t}^{\beta} \neq \Delta\right\}$ and appeal to Lemma 2.1(c) to conclude that on $\left\{N_{\underline{t}}^{\beta} \neq \Delta\right\}$,

$$
\begin{aligned}
P\left(\bar{Z}^{\beta}(b) \leqslant\right. & \left.c \phi_{\alpha}^{(1)}(b) \forall b \in \bar{R}_{n} \mid \mathscr{F}_{1}(\beta)\right) \\
& \leqslant E_{0}^{0}\left(\exp \left\{-\sum_{j=j_{n}+1}^{j_{n+1}} I\left(G^{(\mu)}\left(b_{j}\right)>\left(c / c^{(11)}\right) 2^{j}\right) c^{(12)} G^{(\mu)}\left(b_{j}\right) 2^{-j}\right\}\right),
\end{aligned}
$$

where

$$
G^{(\mu)}\left(b_{j}\right)=\int_{\left(b_{j}^{\alpha}, b_{j}^{\alpha / 2}\right]} p\left(Y_{\underline{s}} / \underline{s}^{1 / \alpha}\right) \underline{s}^{-1} d \lambda(\underline{s})
$$


Let $G(j)=\int_{\alpha 2^{j-1}}^{\alpha 2^{j}} p\left(\tilde{Y}_{u}\right) d u$. A routine weak convergence argument shows there is a sequence $\left\{\eta_{3}(n, \mu): n, \mu \in \mathbf{N}\right\}$ such that $\lim _{\mu \rightarrow \infty} \eta_{3}(n, \mu)=0$ for each $n$ and

$$
\begin{aligned}
& P\left(\bar{Z}^{\beta}(b) \leqslant c \phi_{\alpha}^{(1)}(b) \forall b \in \bar{R}_{n} \mid \mathscr{F}_{1}(\beta)\right) \\
& \quad \leqslant E_{0}^{0}\left(\exp \left\{-\sum_{j=j_{n}+1}^{j_{n+1}} I\left(G(j)>\left(c / c^{(11)}\right) 2^{j}\right) c^{(12)} G(j) 2^{-j}\right\}\right)+\eta_{3}(n, \mu) \\
& \quad \equiv \lambda_{n}^{(2)}+\eta_{3}(n, \mu) .
\end{aligned}
$$

As it stands $\lambda_{n}^{(2)}$ and $\eta_{3}$ also depend on $c$ but $c$ will be fixed below. The Ergodic Theorem (recall $\tilde{Y}_{u}$ is stationary ergodic under $P_{0}^{0}$ ) implies that

$$
G(j) 2^{-j} \stackrel{P}{\rightarrow} m_{0} \equiv(\alpha / 2) E_{0}^{0}\left(p\left(Y_{1}\right)\right) \quad\left(\text { under } P_{0}^{0}\right) \text { as } j \rightarrow \infty .
$$

Now fix $c=K^{(2)} \equiv c^{(11)} m_{0} / 2$. Then

$$
I\left(G(j)>\left(K^{(2)} / c^{(11)}\right) 2^{j}\right) c^{(12)} G(j) 2^{-j} \stackrel{P}{\rightarrow} c^{(12)} m_{0}>0 \quad \text { as } j \rightarrow \infty
$$

and an elementary argument shows

$$
\sum_{j=j_{n}+1}^{j_{n+1}} I\left(G(j)>\left(K^{(2)} / c^{(11)}\right) 2^{j}\right) c^{(12)} G(j) 2^{-j} \stackrel{P}{\rightarrow} \infty \quad \text { as } j \rightarrow \infty .
$$

Dominated convergence implies $\lim _{n \rightarrow \infty} \lambda_{n}^{(2)}=0$. By redefining $\lambda_{n}^{(2)}$ and $\eta_{3}$ (if necessary) we may assume (5.14) without loss of generality. The result is now immediate from (5.15).

THEOREM 5.9. There is a positive sequence $\left\{\lambda_{n}^{(3)}\right\}$ converging to zero, a sequence of natural numbers $\left\{\mu_{n}^{1}\right\}$, and for each $M, N \in \mathbf{N}$, an $n_{1}=n_{1}(M, N)$ such that

$$
P\left(N_{t}\left(S_{1}\left(N_{t}, \bar{R}_{n}, K^{(2)} \phi_{\alpha}^{(1)}\right)\right) \geqslant M \lambda_{n}^{(3)}, \underline{t}<T_{1}(M)\right) \leqslant b_{j_{n+1}}^{N},
$$

whenever $n \geqslant n_{1}, \mu \geqslant \mu_{n}^{1}$ and $\underline{t} \in T \cap\left(b_{j_{n}}^{\alpha / 2}, M\right]$.

Proof. The proof is identical to that of Theorem 5.5 (use Lemma 5.8 in place of 5.4) except for one minor complication. Define $W_{t}(n)$ and $\bar{W}_{t}(n)$ as in the proof of Theorem 5.5 but with $\bar{R}_{n}$ in place of $R_{n}, K^{(2)} \phi_{\alpha}^{(1)}$ in place of $K^{(1)} \phi_{\alpha}$, and $\bar{Z}^{\beta}$ in place of $Z^{\beta}$. Proceed just as in the proof of Theorem 5.5 to obtain (in place of (5.9))

$$
E\left(\left(W_{t}(n)-\bar{W}_{t}(n)\right)^{2 p}\right) \leqslant 32^{p} b_{j_{n}}^{p \alpha / 2} p ! E\left(N_{t}\left(\mathbf{R}^{d}\right)^{p}\right) .
$$

If $\theta=\theta(M)$ satisfies $32 \theta^{2}=(2 M)^{-1}$, then

$$
\begin{gathered}
E\left(\exp \left\{\left|W_{t}(n)-\bar{W}_{t}(n)\right| \theta b_{j_{n}}^{-\alpha / 4}\right\}\right) \leqslant 2 E\left(\cosh \left(\left(W_{t}(n)-\bar{W}_{t}(n)\right) \theta b_{j_{n}}^{-\alpha / 4}\right)\right) \\
\leqslant 2 \sum_{p=0}^{\infty}\left(32 \theta^{2}\right)^{p} p !(2 p !)^{-1} E\left(N_{t}\left(\mathbf{R}^{d}\right)^{p}\right) \quad(\text { by }(5.16) \\
\leqslant 2 E\left(\exp \left((2 M)^{-1} N_{t}\left(\mathbf{R}^{d}\right)\right)\right)=c_{1}(M)<\infty
\end{gathered}
$$

(the last by Proposition 2.6(c) and $\underline{t} \leqslant M, m\left(\mathbf{R}^{d}\right) \leqslant M$; the latter without loss of generality). Therefore

$$
P\left(W_{t}(n)-\bar{W}_{t}(n) \geqslant n^{-2}\right) \leqslant c_{1}(M) \exp \left\{-\theta n^{-2} b_{j_{n}}^{-\alpha / 4}\right\} \leqslant\left(b_{j_{n+1}}\right)^{N}
$$


if $n \geqslant n_{1}=n_{1}(M, N)$, as an easy computation shows. The rest of the proof of Theorem 5.5 now goes through with only obvious changes.

6. Lower bound on $X_{t}$-interpolation arguments and proof. We want to use the probabilistic bound on $N_{t}\left(S_{1}\left(N_{t}, R_{n}, K^{(1)} \phi_{\alpha}\right)\right) \equiv \tilde{N}(\underline{t}, n)$ obtained in Theorem 5.5 to get a bound on $\sup _{\underline{t} \in J} \tilde{N}(\underline{t}, n)$ for some large interval $J$. As in $\S 4$ we divide $J$ into subintervals of length $\Delta_{n}$ and use Theorem 5.5 to show $\sup _{i \Delta_{n} \in J} \tilde{N}\left(i \Delta_{n}, n\right)$ is small. Again there is the problem of interpolating between grid points. Recall that $\tilde{N}(\underline{t}, n)$ is (roughly) the measure of the particles that are the centers of fairly empty or "low density" balls. If $\tilde{N}\left(i \Delta_{n}, n\right)$ is small but $\tilde{N}\left(\underline{t}_{0}, n\right)$ is large for some $\underline{t}_{0} \in$ $\left[i \Delta_{n},(i+1) \Delta_{n}\right)$ then on $\left[i \Delta_{n}, \underline{t}_{0}\right)$ one of three things must have happened (see (6.8)):

1. The low density balls at $i \Delta_{n}$ have suddenly increased in population;

2. A lot of high density balls at $i \Delta_{n}$ have diffused themselves by travelling a relatively large distance in a short time $\left(<\Delta_{n}\right)$; or

3. A lot of high density balls at $i \Delta_{n}$ have diffused themselves by having a large number of particles die in a short time $\left(<\Delta_{n}\right)$.

Lemmas 4.1 and 4.2 will bound the probabilities of these three occurrences. Note that in practice $\underline{t}_{0}$ will be a stopping time and hence we are dealing with $N_{t}$ before a stopping time instead of after it (as in \$4). This is why some stronger estimates are needed (e.g. Lemma 4.2(c) instead of 4.2(a)). There should be a way to work on $\left[\underline{t}_{0},(i+1) / \Delta_{n}\right)$ as before but this argument has eluded us.

Both $\mu \in \mathbf{N}$ and $m=N_{0}^{(\mu)} \in M_{F}^{\mu}$ will vary in this section but dependence on $\mu$ is often suppressed in our notation. We continue to use the notation $a_{j}=2^{-j / \alpha}$, $j_{n}=2^{n}$ and $R_{n}$ from $\S 5$.

Lemma 6.1. Let $R \subset(0,1]$ be finite, $a_{1}=\min R, f: R \rightarrow(0,1]$ be nondecreasing, $\varepsilon \in\left[f\left(a_{1}\right), 1\right]$ and $M \in \mathbf{N} . \exists c^{(13)}(M), c^{(14)}(M)$ and for each $\Delta_{0} \in\left(0, c^{(13)} a_{1}^{\alpha} f\left(a_{1}\right)\right]$, $\exists \mu_{3}=\mu_{3}\left(f(R), \Delta_{0}\right)$ such that if $\mu \geqslant \mu_{3}(\mu \in \mathbf{N})$ and $m \in M_{F}^{\mu}$ satisfies

$$
m\left(S_{1}(m, R, f)\right)<\varepsilon / 16,
$$

then

$$
\begin{array}{r}
P^{m}\left(\sup _{\underline{t}<\Delta_{0} \wedge T_{1}(M)} N_{t}\left(S_{1}\left(N_{t}, 6 R, f(\cdot / 6) / 2\right)\right)>\varepsilon\right) \\
\leqslant c^{(14)} \varepsilon^{-1}\left(\Delta_{0}^{2} f\left(a_{1}\right)^{-2} a_{1}^{-\alpha}+\exp \left\{-f\left(a_{1}\right) / 32 \Delta_{0}\right\}\right) .
\end{array}
$$

Proof. Let $c_{1}>1 / 8$ satisfy $P_{0}^{0}(|Y(\Delta)|>a / 2)<c_{1} \Delta a^{-\alpha} \forall a, \Delta>0$ and define $c^{(13)}(M)=\left(c_{1} 32 M\right)^{-1}$. Let $R, a_{1}, f, \varepsilon$ and $M$ be as above, and let $\Delta_{0} \in$ $\left(0, c^{(13)}(M) a_{1}^{\alpha} f\left(a_{1}\right)\right]$. The definition of $c^{(13)}$ implies (recall $c_{1}>1 / 8$ )

$$
f(a) / 4>\left(8 M P_{0}^{0}\left(\left|Y\left(\Delta_{0}\right)\right|>a / 2\right)\right) \vee \Delta_{0} \quad \forall a \in R .
$$

Finally assume

$$
\mu \geqslant \mu\left(f(R), \Delta_{0}\right) \equiv \max \left\{\mu_{1}\left(f(a) / 4, \Delta_{0}\right): a \in R\right\} \vee \Delta_{0}^{-1},
$$

where $\mu_{1}$ is as in Lemma $4.2(\mathrm{c})$. Let $m \in M_{F}^{\mu}$ satisfy (6.1) and (without loss of generality) $m\left(\mathbf{R}^{d}\right) \leqslant M$. (The probability in question is zero if $m\left(\mathbf{R}^{d}\right)>M$.) 
To simplify the notation write $\bar{S}$ for $\operatorname{supp}(m)-S_{1}(m, R, f)$. We claim there are finite sequences $\left\{y_{i}: i=1, \ldots, N\right\}$ in $\bar{S}$ and $\left\{a^{(i)}: i=1, \ldots, N\right\}$ in $R$ such that

$$
\begin{gathered}
m\left(B\left(y_{i}, a^{(i)}\right)\right) \geqslant f\left(a^{(i)}\right), \\
\bar{S} \subset \bigcup_{i=1}^{N} B\left(y_{i}, 2 a^{(i)}\right), \\
\left\{B\left(y_{i}, a^{(i)}\right): i \leqslant N\right\} \text { are mutually disjoint. }
\end{gathered}
$$

If $a \in R$, let

$$
S(a)=\{y \in \operatorname{supp}(m): m(B(y, a)) \geqslant f(a)\}
$$

Also let $R=\left\{a_{1}, \ldots, a_{K}\right\}$ where $a_{i}<a_{i+1}$. First cover $S\left(a_{K}\right)$ by a finite number of balls of radius $2 a_{K}$ centered in $S\left(a_{K}\right)$ so that the corresponding balls of radius $a_{K}$ form a disjoint set. This may be easily done inductively by simply adding $B\left(y, 2 a_{K}\right)$ if $y \in S\left(a_{K}\right)$ is not yet covered by the previous set of balls of radius $2 a_{K}$. Next proceed inductively through $S\left(a_{K-1}\right)$. If $y \in S\left(a_{K-1}\right)$ is not yet covered by the balls of radius $2 a_{K}$ (that covered $S\left(a_{K}\right)$ ) or the previous set of balls of radius $2 a_{K-1}$ (that we are adding to cover $\left.S\left(a_{K-1}\right)\right)$, then add $B\left(y, 2 a_{K-1}\right)$. Note that $a_{K-1}<a_{K}$ guarantees that $B\left(y, a_{K-1}\right)$ will be disjoint from the disjoint balls of radius $a_{K}$ corresponding to the cover of $S\left(a_{K}\right)$. Continue until we cover $S\left(a_{K}\right) \cup S\left(a_{K-1}\right)$. The obvious induction argument now leads to the required cover of $\bigcup_{i=1}^{K} S\left(a_{i}\right)=\bar{S}$.

If $\beta \sim \underline{t}, N_{0}^{\beta} \in \bar{S}$ and $\left|N_{t}^{\beta}-N_{0}^{\beta}\right| \leqslant a_{1}$, then $N_{0}^{\beta} \in B\left(y_{i}, 2 a^{(i)}\right)$ for some $i \leqslant N$ and hence $N_{t}^{\beta} \in B\left(y_{i}, 3 a^{(i)}\right)$. Therefore if $S(\underline{t}) \equiv S_{1}\left(N_{t}, 6 R, f(\cdot / 6) / 2\right)$, then

$$
\begin{aligned}
N_{\underline{t}}(S(\underline{t})) \leqslant & \mu^{-1} \sum_{\beta \sim \underline{t}} I\left(N_{0}^{\beta} \in S_{1}(m, R, f), N_{\underline{t}}^{\beta} \neq \Delta\right) \\
& +\mu^{-1} \sum_{\beta \sim \underline{t}} I\left(\left|N_{\underline{t}}^{\beta}-N_{0}^{\beta}\right|>a_{1}, N_{\underline{t}}^{\beta} \neq \Delta\right) \\
& +\sum_{i=1}^{N} N_{\underline{t}}\left(B\left(y_{i}, 3 a^{(i)}\right) \cap S(\underline{t})\right) .
\end{aligned}
$$

If $N_{\underline{t}}\left(B\left(y_{i}, 3 a^{(i)}\right) \cap S(\underline{t})\right)>0$ then $N_{\underline{t}}^{\beta} \in S(\underline{t}) \cap B\left(y_{i}, 3 a^{(i)}\right)$ for some $\beta \sim \underline{t}$ and

$$
\begin{aligned}
N_{\underline{t}}\left(B\left(y_{i}, 3 a^{(i)}\right)\right) & \leqslant N_{\underline{t}}\left(B\left(N_{\underline{t}}^{\beta}, 6 a^{(i)}\right)\right)<f\left(a^{(i)}\right) / 2 \\
& \leqslant m\left(B\left(y_{i}, a^{(i)}\right)\right) / 2 \quad(\text { by }(6.4)) .
\end{aligned}
$$

The last summation in (6.7) therefore is bounded by

$$
\frac{1}{2} \sum_{i=1}^{N} m\left(B\left(y_{i}, a^{(i)}\right)\right) I\left(N_{t}\left(B\left(y_{i}, 3 a^{(i)}\right)\right)<f\left(a^{(i)}\right) / 2\right) .
$$


This and (6.7) show that

$$
\begin{aligned}
& P^{m}\left(\sup _{\underline{t}<\Delta_{0} \wedge T_{1}(M)} N_{t}(S(\underline{t}))>\varepsilon\right) \\
& \leq P^{m}\left(\sup _{\underline{t}<\Delta_{0}} \mu^{-1} \sum_{\beta \sim t} I\left(N_{0}^{\beta} \in S_{1}(m, R, f), N_{\underline{t}}^{\beta} \neq \Delta\right)>\varepsilon / 4\right) \\
&+P^{m}\left(\sup _{\underline{t}<\Delta_{0} \wedge T_{1}(M)} \mu^{-1} \sum_{\beta \sim t} I\left(\left|N_{t}^{\beta}-N_{0}^{\beta}\right|>a_{1}, N_{\underline{t}}^{\beta} \neq \Delta\right)>\varepsilon / 4\right) \\
&+P^{m}\left(\frac{1}{2} \sum_{i=1}^{N} m\left(B\left(y_{i}, a^{(i)}\right)\right)\right. \\
&\left.\quad \times I\left(\inf _{\underline{t}<\Delta_{0} \wedge T_{1}(M)} N_{t}\left(B\left(y_{i}, 3 a^{(i)}\right)\right)<f\left(a^{(i)}\right) / 2\right)>\varepsilon / 2\right) \\
& \equiv \mathrm{I}+\mathrm{II}+\mathrm{III} . \quad
\end{aligned}
$$

If

$$
p_{i}=P^{m}\left(\inf _{\underline{t}<\Delta_{0} \wedge T_{1}(M)} N_{t}\left(B\left(y_{i}, 3 a^{(i)}\right)\right)<f\left(a^{(i)}\right) / 2\right),
$$

then an elementary argument shows

$$
\begin{aligned}
p_{i} \leqslant & P^{m}\left(\sup _{\underline{t}<T_{1}(M) \wedge \Delta_{0}} \mu^{-1} \sum_{\beta \sim t} I\left(\left|N_{t}^{\beta}-N_{0}^{\beta}\right|>2 a^{(i)}\right)>f\left(a^{(i)}\right) / 4\right) \\
& +P^{m}\left(\inf _{\underline{t}<\Delta_{0}} \mu^{-1} \sum_{\beta \sim t} I\left(N_{0}^{\beta} \in B\left(y_{i}, a^{(i)}\right), N_{t}^{\beta} \neq \Delta\right)<(3 / 4) f\left(a^{(i)}\right)\right. \\
& \\
\leqslant & c^{(7)} 16 f\left(a^{(i)}\right)^{-2} M \Delta_{0} P_{0}^{0}\left(\left|Y\left(\Delta_{0}\right)\right|>a^{(i)}\right) \\
& +\exp \left\{-f\left(a^{(i)}\right) / 32 \Delta_{0}\right\}+\eta_{1}\left(f\left(a^{(i)}\right), \Delta_{0}, 1 / 4, \mu\right) .
\end{aligned}
$$

In the last line we have used Lemmas 4.2(c) and 4.1(a). (6.4) is used to apply the latter and (6.3) allows us to use the former. If

$$
\eta\left(f(R), \Delta_{0}, \mu\right)=\max _{a \in R} \eta_{1}\left(f(a), \Delta_{0}, 1 / 4, \mu\right),
$$

then for some $c_{2}<\infty$,

$$
p_{i} \leqslant c_{2} M \Delta_{0}^{2} a_{1}^{-\alpha} f\left(a_{1}\right)^{-2}+\exp \left\{-f\left(a_{1}\right) / 32 \Delta_{0}\right\}+\eta .
$$

Therefore

$$
\begin{aligned}
\mathrm{III} & \leqslant \varepsilon^{-1}\left(\sum_{i=1}^{N} m\left(B\left(y_{i}, a^{(i)}\right)\right)\right)\left(c_{2} M \Delta_{0}^{2} a_{1}^{-\alpha} f\left(a_{1}\right)^{-2}+\exp \left\{-f\left(a_{1}\right) / 32 \Delta_{0}\right\}+\eta\right) \\
& \leqslant \varepsilon^{-1} M\left(c_{2} M \Delta_{0}^{2} a_{1}^{-\alpha} f\left(a_{1}\right)^{-2}+\exp \left\{-f\left(a_{1}\right) / 32 \Delta_{0}\right\}+\eta\right)
\end{aligned}
$$


(by (6.6) and $m\left(\mathbf{R}^{d}\right) \leqslant M$ ). Use Lemmas $4.1(\mathrm{~b})$ and 4.2 (c) to bound I and II, respectively, and hence conclude from (6.8) and the above that

$$
\begin{aligned}
P^{m}\left(\sup _{\substack{t<\Delta_{0} \wedge T_{1}(M) \\
N_{t}}} N_{t}(S(\underline{t}))>\varepsilon\right) \\
\leqslant \exp \left\{-\left(\Delta_{0}+\mu^{-1}\right)^{-1}(\varepsilon / 8-\varepsilon / 16)\right\}+c^{(7)} 16 \varepsilon^{-2} M \Delta_{0} P_{0}^{0}\left(\left|Y\left(\Delta_{0}\right)\right|>a_{1} / 2\right) \\
\quad+\varepsilon^{-1} M\left(c_{2} M \Delta_{0}^{2} a_{1}^{-\alpha} f\left(a_{1}\right)^{-2}+\exp \left\{-f\left(a_{1}\right) / 32 \Delta_{0}\right\}+\eta\right) .
\end{aligned}
$$

In applying Lemma 4.1(b) we have used (6.1), and (6.3) allows us to use Lemma 4.2(c) (recall $\left.\varepsilon / 4 \geqslant f\left(a_{1}\right) / 4\right)$. This last observation and $\mu \geqslant \mu\left(f(R), \Delta_{0}\right) \geqslant \Delta_{0}^{-1}$ show

$$
\begin{aligned}
& P^{m}\left(\sup _{\underline{t}<\Delta_{0} \wedge T_{1}(M)} N_{t}(S(\underline{t}))>\varepsilon\right) \\
& \leqslant c_{3}(M) \varepsilon^{-1}\left[\exp \left(-f\left(a_{1}\right) / 32 \Delta_{0}\right)+f\left(a_{1}\right)^{-2} \Delta_{0}^{2} a_{1}^{-\alpha}+\eta\right]
\end{aligned}
$$

Choose $\mu_{3}\left(f(R), \Delta_{0}\right) \geqslant \mu\left(f(R), \Delta_{0}\right)$ such that $\eta\left(f(R), \Delta_{0}, \mu\right) \leqslant \exp \left(-f\left(a_{1}\right) / 32 \Delta_{0}\right)$ for $\mu \geqslant \mu_{3}$; the right side of (6.9) is then bounded by (6.2) for such a $\mu$.

Notation. Let $T_{2}(M)=T_{1}(M) \wedge M$ for $M>0$.

THEOREM 6.2. There is a sequence $\left\{\mu_{n}\right\}$ in $\mathbf{N}$ and positive doubly indexed sequences $\left\{\varepsilon_{n, M}\right\},\left\{\lambda_{n, M}\right\}$ such that $\lim _{n \rightarrow \infty} \lambda_{n, M}=0$ and $\sum_{n=1}^{\infty} \varepsilon_{n, M}<\infty \forall M \in \mathbf{N}$, and if $\mu \geqslant \mu_{n}, m \in M_{F}^{\mu}$ and $M \in \mathbf{N}$, then

$$
P^{m}\left(\sup _{4 a_{j_{n}}^{\alpha}<t<T_{2}(M), t \in T} N_{t}\left(S_{1}\left(N_{\underline{t}}, 6 R_{n},\left(K^{(1)} / 2\right) \phi_{\alpha}(\cdot / 6)\right)\right)>\lambda_{n, M}\right) \leqslant \varepsilon_{n, M} .
$$

PROOF. Let $\lambda_{n, M}=\left(\left(16 \lambda_{n}^{1}\right) \vee n^{-2}\right) M\left(\lambda_{n}^{1}\right.$ as in Theorem 5.5), $\Delta_{n}=a_{j_{n+1}}^{3 \alpha+1}, \underline{\Delta}_{n}=$ $\left[\mu \Delta_{n}\right] / \mu$, and define $\mu_{n}=\mu_{n}^{0} \vee \mu_{3}\left(K^{(1)} \phi_{\alpha}\left(R_{n}\right), \Delta_{n}\right)\left(\mu_{n}^{0}\right.$ as in Theorem 5.5 and $\mu_{3}$ as in Lemma 6.1). Choose $M \in \mathbf{N}$, let $N \in \mathbf{N}, N \geqslant 3 \alpha+2$, and by making $n_{0}(M, N)$ (as in Theorem 5.5) larger, if necessary, assume that for $n \geqslant n_{0}$

$$
\begin{gathered}
\Delta_{n} \leqslant c^{(13)} a_{j_{n+1}^{\alpha}}^{\alpha} K^{(1)} \phi_{\alpha}\left(a_{j_{n+1}}\right)<2 a_{j_{n}}^{\alpha}, \\
n^{-2} \geqslant K^{(1)} \phi_{\alpha}\left(a_{j_{n+1}}\right) \geqslant K^{(1)} a_{j_{n+1}}^{\alpha}, \quad \lambda_{n, M} \leqslant 1 .
\end{gathered}
$$

Fix natural numbers $n \geqslant n_{0}$ and $\mu \geqslant \mu_{n}$. Note that $\mu \geqslant \mu_{3} \geqslant \Delta_{n}^{-1}$ (by definition) so that $\underline{\Delta}_{n}>0$. Then

$$
\begin{aligned}
& P^{m}\left(\sup _{4 a_{j_{n}}^{\alpha}<t<T_{2}(M)} N_{t}\left(S_{1}\left(N_{t}, 6 R_{n},\left(K^{(1)} / 2\right) \phi_{\alpha}(\cdot / 6)\right)\right)>\lambda_{n, M}\right) \\
& \leqslant \sum_{i: 2 a_{j_{n}}^{\alpha}<i \underline{\Delta}_{n}<M} E^{m}\left(I\left(i \underline{\Delta}_{n}<T_{1}(M)\right)\right. \\
& \left.\quad \times P^{N_{i \Delta_{n}}}\left(\sup _{0 \leqslant i<\underline{\Delta}_{n} \wedge T_{i}(M)} N_{t}\left(S_{1}\left(N_{t}, 6 R_{n},\left(K^{(1)} / 2\right) \phi_{2}(\cdot / 6) \phi_{\alpha} / 2\right)\right)>\lambda_{n, M}\right)\right)
\end{aligned}
$$

(by the Markov property and (6.10)) 
(continued)

$$
\begin{aligned}
\leqslant & \sum_{2 a_{j_{n} \underline{\Delta}_{n}^{-1}<i<M \underline{\Delta}_{n}^{-1}}} E^{m}\left(I\left(i \underline{\Delta}_{n}<T_{1}(M), N_{i \underline{\Delta}_{n}}\left(S_{1}\left(N_{i \underline{\Delta}_{n}}, R_{n}, K^{(1)} \phi_{\alpha}\right)\right)<\lambda_{n, M} / 16\right)\right. \\
& \left.\times P^{N_{i \underline{\Delta}_{n}}}\left(\sup _{t \in \underline{\Delta}_{n} \wedge T_{1}(M)} N_{\underline{t}}\left(S_{1}\left(N_{\underline{t}}, 6 R_{n},\left(K^{(1)} / 2\right) \phi_{\alpha}(\cdot / 16)\right)\right)>\lambda_{n, M}\right)\right) \\
& +P^{m}\left(i \underline{\Delta}_{n}<T_{1}(M), N_{i \underline{\Delta}_{n}}\left(S_{1}\left(N_{i \underline{\Delta}_{n}}, R_{n}, K^{(1)} \phi_{\alpha}\right)\right) \geqslant \lambda_{n, M} / 16 \geqslant M \lambda_{n}^{1}\right) \\
\leqslant & M \underline{\Delta}_{n}^{-1}\left[c ^ { ( 1 4 ) } \lambda _ { n , M } ^ { - 1 } \left(\underline{\Delta}_{n}^{2}\left(K^{(1)} \phi_{\alpha}\left(a_{j_{n+1}}\right)\right)^{-2} a_{j_{n+1}-\alpha}\right.\right. \\
& \left.\left.+\exp \left\{-K^{(1)} \phi_{\alpha}\left(a_{j_{n+1}}\right) / 32 \underline{\Delta}_{n}\right\}\right)+a_{j_{n+1}}^{N}\right] .
\end{aligned}
$$

In the last line (6.10) and (6.11) allow us to apply Lemma 6.1 and we have also used Theorem 5.5. Use (6.11) and the definitions of $\lambda_{n, M}$ and $\Delta_{n}\left(\leqslant 2 \underline{\Delta}_{n}\right)$ to bound the above by

$$
c^{(14)}\left[n^{2} K^{(1)^{-2}} a_{j_{n+1}}+2 M a_{j_{n+1}}^{-3 \alpha-1} \exp \left\{-K^{(1)} a_{j_{n+1}}^{-2 \alpha-1} / 32\right\}\right]+M 2 a_{j_{n+1}} \equiv \varepsilon_{n, M} .
$$

Clearly $\sum_{n} \varepsilon_{n, M}<\infty$ and the proof is complete.

To complete the proof of the lower bound on $X_{t}$ we again work in an $\omega_{1}$-saturated enlargement of a superstructure containing our original $(\Omega, \mathscr{A})$.

TheOREM 6.3. Assume $d \geqslant \alpha$. There is a positive constant $c$, depending only on $(\alpha, d)$, such that for any $m \in M_{F}$, and $Q^{m}$-a.a. $\omega$ :

$$
\begin{aligned}
& \forall t>0 \exists \Lambda_{t}(\omega) \in \mathscr{B}\left(\mathbf{R}^{d}\right) \text { such that } X_{t}\left(\Lambda_{t}^{c}\right)=0 \text { and } \\
& X_{t}\left(A \cap \Lambda_{t}\right) \geqslant c \phi_{\alpha}-m\left(A \cap \Lambda_{t}\right) \text { for all } A \in \mathscr{B}\left(\mathbf{R}^{d}\right) .
\end{aligned}
$$

Proof. Choose $m \in M_{F}$. Let $N_{t}=N_{t}^{\mu}\left(\mu \in{ }^{*} \mathbf{N}-\mathbf{N}\right)$ be the internal $S$-continuous lifting of $X_{t}(=\operatorname{st}(N)(t))$ constructed in the proof of Theorem 4.5 and defined on the appropriate Loeb space $(* \Omega, \mathscr{F}, P)$. Choose $M \in \mathbf{N}$ and let

$$
\tau_{2}(M)=\inf \left\{t \geqslant 0 \mid X_{t}\left(\mathbf{R}^{d}\right) \geqslant M\right\} \wedge M .
$$

Argue just as in the proof of Theorem 4.5 to get

$$
\begin{aligned}
& P\left(\sup _{4 a_{j_{n}}^{\alpha}<t<\tau_{2}(M)} X_{t}\left(S_{1}\left(X_{t}, 12 R_{n},\left(K^{(1)} / 4\right) \phi_{\alpha}(\cdot / 12)\right)\right) \geqslant 2 \lambda_{n, M}\right) \\
& \quad \leqslant{ }^{*} P^{m^{(\mu)}}\left(\sup _{4 a_{j_{n}}^{\alpha}<t<T_{2}(M)} N_{t}\left(S_{1}\left(N_{t}, 6 R_{n},\left(K^{(1)} / 2\right) \phi_{\alpha}(\cdot / 6)\right)\right) \geqslant \lambda_{n, M}\right) \\
& \leqslant \varepsilon_{n, M}
\end{aligned}
$$

by Theorem $6.2\left(\mu \geqslant \mu_{n}\right)$. By Borel-Cantelli $\left(\sum \varepsilon_{n, M}<\infty\right) \exists N(\omega)<\infty$ a.s. such that if $n \geqslant N(\omega)$ then

$$
\begin{aligned}
X_{t}\left(\left\{x: X_{t}\left(B\left(x, 12 a_{j}\right)\right)<\left(K^{(1)} / 4\right) \phi_{\alpha}\left(a_{j}\right) \forall j \in\left(j_{n}, j_{n+1}\right]\right\}\right)<2 \lambda_{n, M} \\
\forall t \in\left[M^{-1}, \tau_{2}(M)\right) .
\end{aligned}
$$

Choose a subsequence such that $\sum_{i=1}^{\infty} \lambda_{n_{i}, M}<\infty$ and fix $\omega$ such that $N(\omega)<\infty$. Let $t \in\left[M^{-1}, \tau_{2}(M)(\omega)\right)$. Applying Borel-Cantelli with respect to $X_{t}(\omega)$, one gets an $i_{0}(t, x)<\infty X_{t}$-a.s. such that

$$
X_{t}\left(B\left(x, 12 a_{j}\right)\right) \geqslant\left(K^{(1)} / 4\right) \phi_{\alpha}\left(a_{j}\right) \text { for some } j \in\left(j_{n_{i}}, j_{n_{i+1}}\right] \text { and all } i \geqslant i_{0} \text {, }
$$


and hence

$$
\underset{a \downarrow 0}{\limsup } X_{t}(B(x, 12 a)) \phi_{\alpha}(a)^{-1} \geqslant K^{(1)} / 4 \text { for } X_{t} \text {-a.a. } x \text {. }
$$

Define

$$
\Lambda_{t}(\omega)=\left\{x: \limsup _{a \downarrow 0} X_{t}(B(x, a)) \phi_{\alpha}(a)^{-1} \geqslant K^{(1)} 4^{-1} 12^{-\alpha}\right\} .
$$

Then $X_{t}\left(\Lambda_{t}^{c}\right)=0$ for $t \in\left[1 / M, \tau_{2}(M)\right)$ a.s. and hence for all $t>0$ a.s. by letting $M \rightarrow \infty$. Apply Theorem 1.4 with $\nu=X_{t}(\omega)$ to complete the proof.

Virtually the same argument gives a slightly stronger result if $d=\alpha$ (use Theorem 5.9 instead of Theorem 5.5 to first prove an analogue of Theorem 6.2).

THEOREM 6.4. Assume $d=\alpha$. There is a positive constant $c$, depending only on $(\alpha, d)$ such that for all $m \in M_{F}$ and $Q^{m}$-a.a. $\omega$ :

$$
\begin{aligned}
& \forall t>0 \exists \Lambda_{t}(\omega) \in \mathscr{B}\left(\mathbf{R}^{d}\right) \text { such that } X_{t}\left(\Lambda_{t}^{c}\right)=0 \text { and } \\
& X_{t}\left(A \cap \Lambda_{t}\right) \geqslant c \phi_{\alpha}^{(1)}-m\left(A \cap \Lambda_{t}\right) \text { for all } A \in \mathscr{B}\left(\mathbf{R}^{d}\right) .
\end{aligned}
$$

The following is an immediate consequence of the proofs of Theorems 4.5 and 6.3.

TheOrem 6.5. Assume $d>\alpha$. There are constants $c$, $C$ satisfying $0<c \leqslant C<\infty$, and depending only on $(\alpha, d)$, such that for any $m \in M_{F}$ and $Q^{m}-a . a$. $\omega$

$$
c \leqslant \limsup X_{t}(B(x, a)) \phi_{\alpha}(a)^{-1} \leqslant C \text { for } X_{t} \text {-a.a. } x \text { and all } t>0 .
$$

Similar, but less precise, results are also immediate for $d=\alpha$.

7. Infinite initial measures. The constants in Theorems 4.5, 4.6, 4.7, 6.3, 6.4 and 6.5 do not depend on the initial measure $m$ and so it will come as no surprise that these results extend easily to a large class of infinite initial measures, including Lebesgue measure. One must only be a little careful with the rate of increase of $m(B(0, n))$ as $n \rightarrow \infty$ to avoid particles "cascading in from $\infty$ " in finite time. Most of the work has been done by Iscoe [1986]. We sketch the additional arguments that are needed because we were unable to find them in the literature, even though they must be well known to the experts.

Notation. If $p \geqslant 0$, let

$$
\begin{gathered}
\phi_{p}(x)=\left(1+|x|^{p}\right)^{-1}, \quad M_{p}\left(\mathbf{R}^{d}\right)=\left\{m \in M\left(\mathbf{R}^{d}\right): \int \phi_{p} d m<\infty\right\}, \\
C_{p}\left(\mathbf{R}^{d}\right)=\left\{\phi \in C\left(\mathbf{R}^{d}\right): \phi \phi_{p}^{-1} \text { is bounded }\right\}, C_{p}^{+}\left(\mathbf{R}^{d}\right)=\left\{\phi \in C_{p}\left(\mathbf{R}^{d}\right): \phi \geqslant 0\right\} .
\end{gathered}
$$

Topologize $M_{p}=M_{p}\left(\mathbf{R}^{d}\right)$ so that $m_{n} \rightarrow m$ in $M_{p}$ iff $\lim _{n \rightarrow \infty} \int \phi d m_{n}=\int \phi d m$ for $\phi$ continuous with compact support or $\phi=\phi_{p} . M_{p}$ is a separable metric space (Iscoe [1986, p. 89]). Fix $p \in(d, d+\alpha)$ if $\alpha<2$, and $p \in(d, \infty)$ if $\alpha=2$.

Iscoe [1986] showed there is a unique probability kernel $p_{t}(m, A)\left(t \geqslant 0, m \in M_{p}\right.$, $\left.A \in \mathscr{B}\left(M_{p}\right)\right)$ such that if $m \in M_{p}$ and $\phi \in C_{p}^{+}$, then

$$
\int \exp \{-x(\phi)\} p_{t}(m, d x)=\exp \left\{-\int u(t, x) d m(x)\right\},
$$


where $u$ is the unique solution of (1.2). He also proves there is a homogeneous Feller process with paths in $C\left(M_{p}\right)$, having transition kernel $p_{t}$ (continuity is clear in our more restrictive setting and in fact is reproved below). We call this process the $d$-dimensional branching diffusion of index $\alpha$, suppressing dependence on $p$ (if $p=0$ it is the process introduced in $\S 1)$, and continue to denote its law on $C\left(M_{p}\right)$ by $Q^{m}$, if $X_{0} \equiv m \in M_{p}$.

To extend our results to the $M_{p}$-valued process we show that such a process is a uniform (for $t \leqslant n$ ) limit of the $M_{F}$-valued processes already studied in detail. Let $m \in M_{p}$ and $\left\{A_{n}\right\}$ be a sequence of disjoint, bounded Borel sets whose union is $\mathbf{R}^{d}$. Let $\left\{X_{n}\right\}$ be a collection of independent $\left(M_{F}\right.$-valued) $d$-dimentional branching diffusions of index $\alpha$ such that $X_{n}(0)(A)=m_{n}(A) \equiv m\left(A \cap A_{n}\right) .\left\{X_{n}\right\}$ are defined on a common $\left(\Omega, \mathscr{F}, \mathscr{F}_{t}, P\right)$, on which the conclusion of Theorem 1.1 holds (with $m_{n}$ in place of $m$ ). Appealing to Theorem 1.1, we see that if $m^{(n)}=\sum_{i=1}^{n} m_{i}$ and $X^{(n)}=\sum_{i=1}^{n} X_{i}$, then

$$
\begin{aligned}
& X_{t}^{(n)}(\phi)=m^{(n)}(\phi)+\int_{0}^{t} X_{s}^{(n)}(A \phi) d s+Z_{i}^{(n)}(\phi), \quad \phi \in D(A), \\
& Z_{t}^{(n)}(\phi) \text { is a continuous } L^{2} \mathscr{F}_{t} \text {-martingale, }\left\langle Z^{(n)}(\phi)\right\rangle_{t}=\int_{0}^{t} X_{s}^{(n)}\left(\phi^{2}\right) d s,
\end{aligned}
$$

and hence $X^{(n)}$ is the branching diffusion starting at $m^{(n)}$. By monotonicity we may define an $M\left(\mathbf{R}^{d}\right)$-valued process by

$$
X_{t}(A)=\lim _{n \rightarrow \infty} X_{t}^{(n)}(A), \quad A \in \mathscr{B}\left(\mathbf{R}^{d}\right), t \geqslant 0 .
$$

If $\phi \in C_{p}$ and $0 \leqslant t \leqslant t_{0}$, then Proposition 2.2 and Monotone Convergence imply

$$
\begin{aligned}
E\left(\left|X_{t}(\phi)-X_{t}^{(n)}(\phi)\right|\right) & \leqslant \sum_{m=n+1}^{\infty} \int_{A_{m}} E_{0}^{x}\left(\left|\phi\left(Y_{t}\right)\right|\right) d m(x) \\
& \leqslant C\left(t_{0}\right) \sum_{m=n+1}^{\infty} \int_{A_{m}} \phi_{p}(x) d m(x) \\
& \rightarrow 0 \text { as } n \rightarrow \infty .
\end{aligned}
$$

Let $\phi \in D(A) \cap C_{p}, t_{0}>0$ and $n_{1}<n_{2}$ be natural numbers. Then

$$
\begin{aligned}
E\left(\sup _{0 \leqslant t \leqslant t_{0}}\left(Z_{t}^{\left(n_{2}\right)}(\phi)-Z_{t}^{\left(n_{1}\right)}(\phi)\right)^{2}\right) & \leqslant c_{1} \int_{0}^{t_{0}} E\left(X_{t}^{\left(n_{2}\right)}\left(\phi^{2}\right)-X_{t}^{\left(n_{1}\right)}\left(\phi^{2}\right)\right) d t \\
& \rightarrow 0 \text { as } n_{1}, n_{2} \rightarrow \infty\left(\text { by (7.3) since } \phi^{2} \in C_{p}\right) .
\end{aligned}
$$

Therefore for a subsequence $\left\{n_{k}\right\}$ one has

$$
Z_{t}^{\left(n_{k}\right)}(\phi) \rightarrow Z_{t}(\phi) \text { uniformly for } t \text { in compacts a.s. and in } L^{2} \forall \phi \in C_{p} \cap D(A) \text {. }
$$

The limit $Z_{t}(\phi)$ must be a continuous, $L^{2} \mathscr{F}_{t}$-martingale such that

$$
\langle Z(\phi)\rangle_{t}=\lim _{n \rightarrow \infty}\left\langle Z^{(n)}(\phi)\right\rangle_{t}=\int_{0}^{t} X_{s}\left(\phi^{2}\right) d s .
$$


If, in addition, $A \phi \in C_{p}$, then

$$
\begin{aligned}
\sup _{t \leqslant t_{0}}\left|\int_{0}^{t} X_{s}(A \phi)-X_{s}^{(n)}(A \phi) d s\right| & \leqslant c_{2}(\phi) \int_{0}^{t_{0}}\left(X_{s}-X_{s}^{(n)}\right)\left(\phi_{p}\right) d s \\
& \rightarrow 0 \quad \text { a.s. and in } L^{1}
\end{aligned}
$$

(by (7.3) and monotonicity in $n$ ). (7.4) and (7.5) show that the right side of (7.2) converges uniformly for $\boldsymbol{t}$ in compacts a.s. along the subsequence $\left\{\boldsymbol{n}_{\boldsymbol{k}}\right\}$ and for $\phi$ as above. Therefore if $\phi \in D(A) \cap C_{p}$ satisfies $A \phi \in C_{p}$, then

$$
\left\{\begin{array}{l}
X_{t}(\phi)=m(\phi)+\int_{0}^{t} X_{s}(A \phi) d s+Z_{t}(\phi), \\
Z_{t}(\phi) \text { is a continuous, } L^{2} \mathscr{F}_{t} \text {-martingale such that }\langle Z(\phi)\rangle_{t}=\int_{0} X_{s}\left(\phi^{2}\right) d s .
\end{array}\right.
$$

Recall that $p>d \geqslant 2$, so that $\phi_{p}$ is $C^{2}$ and $\Delta \phi_{p} \in C_{p}$. Therefore $\phi_{p} \in D(A)$ and (Feller [1966, p. 433]) if $T_{t}$ is the Brownian semigroup and $\alpha<2$,

$$
\begin{aligned}
A \phi_{p}(x) & =c_{3} \int_{0}^{\infty}\left(T_{s} \phi_{p}(x)-\phi_{p}(x)\right) s^{-1-\alpha / 2} d s \\
& =c_{3} \int_{0}^{\infty} \int_{0}^{s} T_{u}\left(\Delta \phi_{p}\right)(x) d u s^{-1-\alpha / 2} d s \\
& \leqslant c_{4} \int_{0}^{\infty} s^{-\alpha / 2} \phi_{p}(x) d s \quad \text { (Iscoe [1986, Corollary 2.4]), } \\
A \phi_{p}(x) & \leqslant c_{5} \phi_{p}(x) .
\end{aligned}
$$

If $\alpha=2$ the above conclusion is trivial. Therefore (7.6) holds for $\phi=\phi_{p}$ and hence by comparison for any $\phi \in C_{p}$. In fact the monotonicity in $n$ of

shows that

$$
\sup _{t \leqslant t_{0}}\left|X_{t}\left(\phi_{p}\right)-X_{t}^{(n)}\left(\phi_{p}\right)\right|
$$

$$
\sup _{t \leqslant t_{0}}\left|X_{t}(\phi)-X_{t}^{(n)}(\phi)\right| \rightarrow 0 \quad \text { a.s. and in } L^{1} \text { as } n \rightarrow \infty \forall \phi \in C_{p} .
$$

$X_{t}(\phi)$ is therefore a continuous $M_{p}$-valued process. As in Iscoe [1986], one can now easily check that $X_{t}$ is a homogeneous Markov process whose transition kernel is given by (7.1). (Alternatively one can show that (7.7) characterizes this process.) We have proved

Theorem 7.1. Let $d<p<d+\alpha$ if $\alpha<2$, and $d<p$ if $\alpha=2$. Assume $m \in M_{p}$, $\left\{A_{n} \mid n \in \mathbf{N}\right\}$ is a sequence of disjoint bounded sets in $\mathscr{B}\left(\mathbf{R}^{d}\right)$ such that $\cup A_{n}=\mathbf{R}^{d}$, and $m_{n}(\cdot)=m\left(\cdot \cap A_{n}\right)$. Let $\left\{X_{n}\right\}$ be a sequence of independent d-dimensional branching diffusions of index $\alpha$ defined on a common $\left(\Omega, \mathscr{F}, \mathscr{F}_{t}, P\right)$ and such that $X_{n}(0)=m_{n}$. Let $m^{(n)}=\sum_{j-1}^{n} m_{j}$ and $X^{(n)}=\sum_{j-1}^{n} X_{j}$. Then $X^{(n)}$ is a d-dimensional branching diffusion starting at $m^{(n)}$ and $X_{t}(A)=\lim _{n \rightarrow \infty} X_{t}^{(n)}(A)$ is a d-dimensional $\left(M_{p^{-}}\right.$ valued) branching diffusion starting at $m$. Moreover (7.7) and (7.8) hold. 
The extensions of Theorems 4.5, 4.6, 4.7, 6.3, 6.4 and 6.5 to the case of an initial measure $m$ in $M_{p}$ are now easy. To illustrate this we only prove

THEOREM 7.2. Assume $\alpha<d$. There are constants $c$, $C$ satisfying $0<c<C<\infty$, and depending only on $(\alpha, d)$ such that if $p$ is as in Theorem 7.1 and $m \in M_{p}$, then for $Q^{m}$-a.a. $\omega$ :

$$
\begin{aligned}
& \forall t>0 \exists \Lambda_{t}(\omega) \in \mathscr{B}\left(\mathbf{R}^{d}\right) \text { such that } X_{t}\left(\Lambda_{t}^{c}\right)=0 \text { and } \\
& c \phi_{\alpha}-m\left(A \cap \Lambda_{t}\right) \leqslant X_{t}(A) \leqslant C \phi_{\alpha}-m\left(A \cap \Lambda_{t}\right) \forall A \in \mathscr{B}\left(\mathbf{R}^{d}\right) .
\end{aligned}
$$

Proof. Let $X^{(n)}$ and $X$ be as in Theorem 7.1. Define $\Lambda_{t}^{(n)}$ by (6.12) but with $X^{(n)}$ in place of $X$. The proof of Theorem 6.3 shows that for some $c>0$ and a.a. $\omega$

$$
\left\{\begin{array}{l}
X_{t}^{(n)}\left(\Lambda_{t}^{(n)^{c}}\right)=0 \quad \forall t>0, \\
X_{t}^{(n)}(A) \geqslant c \phi_{\alpha}-m\left(A \cap \Lambda_{t}^{(n)}\right) \quad \forall t>0, A \in \mathscr{B}\left(\mathbf{R}^{d}\right) .
\end{array}\right.
$$

Fix $\omega$ so that for all $n \in \mathbf{N}$, (7.9) holds and the conclusion of Theorem 4.5 holds with $X^{(n)}$ in place of $X . \Lambda_{t}^{(n)}(\omega) \uparrow \Lambda_{t}(\omega)$ and $\Lambda_{t}$ supports $X_{t}$ by (7.9). If $t>0$ and $A \in \mathscr{B}\left(\mathbf{R}^{d}\right)$, then

$$
\begin{aligned}
c \phi_{\alpha}-m\left(A \cap \Lambda_{t}\right) & =\lim _{n \rightarrow \infty} c \phi_{\alpha}-m\left(A \cap \Lambda_{t}^{(n)}\right) \\
& \leqslant \lim _{n \rightarrow \infty} X_{t}^{(n)}(A)=X_{t}(A) \quad(\text { by }(7.9)) \\
& =\lim _{n \rightarrow \infty} X_{t}^{(n)}\left(A \cap \Lambda_{t}^{(n)}\right) \leqslant C \phi_{\alpha}-m\left(A \cap \Lambda_{t}\right) \quad \text { (Theorem 4.5) }
\end{aligned}
$$

ReMARK. If $\alpha=2$, it is not hard to show that the space of initial measures for $X_{t}$ may be enlarged to

$$
M_{\exp }=\left\{m \in M\left(\mathbf{R}^{d}\right): \int e^{-\varepsilon|x|^{2}} d m(x)<\infty \forall \varepsilon>0\right\} .
$$

$m_{n} \rightarrow m$ in $M_{\exp }$ iff $\int \phi d m_{n} \rightarrow \int \phi d m$ for $\phi$ continuous on compact support and $\phi(x)=e^{-\varepsilon|x|^{2}}$ for each $\varepsilon>0$. There is a unique $M_{\text {exp }}$-valued diffusion satisfying the martingale problem (7.7) for each $m \in M_{\text {exp }}$. $\left(C_{p}\right.$ must be replaced by the obvious dual class of continuous functions.) The analogues of Theorems 7.1 and 7.2 hold. It is not possible to go beyond $M_{\text {exp }}$. If $m \notin M_{\exp }$ and $\left\{X^{(n)}\right\}$ are constructed as in Theorem 7.1, then $X_{t}=\lim _{n \rightarrow \infty} X_{t}^{(n)}$ is continuous on $\left[0, t_{0}\right)$ but $X_{t}(B)=\infty$ for any open ball if $t>t_{0}$, where

$$
\left(2 t_{0}\right)^{-1}=\inf \left(\left\{\varepsilon: \int e^{-\varepsilon|x|^{2}} d m(x)<\infty\right\} .\right.
$$

This is the time it takes for some of the particles at $\infty$ to saturate finite balls.

ADDED IN PROOF. Recent developments have shown that the conclusion of Theorem A continues to hold if $\Lambda_{t}$ is replaced by the closed support of $X_{t}$ and $\alpha=2$, and that this extension to closed supports is false if $0<\alpha<2$. This question was raised in the introduction. 
ACKNOWLEDGment. I thank Don Dawson for telling me about this problem and pointing out several references, and John Walsh for showing me the labelling system used throughout this work and for many helpful discussions on the worm's-eye view of things.

\section{REFERENCES}

R. M. Anderson and S. Rashid (1978), A nonstandard characterization of weak convergence, Proc. Amer. Math. Soc. 69, 327-332.

R. B. Ash (1972), Real analysis and probability, Academic Press, New York.

J. T. Cox and D. Griffeath (1985), Occupation times for critical branching Brownian motions, Ann. Probab. 13, 1108-1132.

N. Cutland (1983), Nonstandard measure theory and its applications, Bull. London Math. Soc. 15, 529-589.

D. A. Dawson (1975), Stochastic evolution equations and related measure processes, J. Multivariate Anal. 5, 1-52.

(1977), The critical measure diffusion process, Z. Wahrsch. Verw. Gebiete 40, 125-145.

D. A. Dawson and K. J. Hochberg (1979), The carrying dimension of a stochastic measure diffusion, Ann. Probab. 7, 693-703.

D. A. Dawson and T. G. Kurtz (1982), Application of duality to measure-valued processes, Lecture Notes in Control and Information Sci., vol. 42 (W. Fleming and L. G. Gorostiza, eds.), Springer-Verlag, New York.

W. Feller (1951), Diffusion processes in genetics, Proc. 2nd Berkeley Sympos., pp. 227-246. (1966), An introduction to probability theory and its applications. Vol. II, Wiley, New York.

T. E. Harris (1963), The theory of branching processes, Springer-Verlag, New York.

J. Hawkes (1971), A lower Lipschitz condition for the stable subordinator, Z. Wahrsch. Verw. Gebiete 17, 23-32.

R. A. Holley, and D. W. Stroock (1978), Generalized Ornstein-Uhlenbeck processes and infinite particle branching Brownian motions, Publ. Res. Inst. Math. Sci. 14, 741-788.

I. Iscoe (1986), A weighted occupation time for a class of measure-valued branching processes, Probab. Th. Rel. Fields 71, 85-116.

F. B. Knight (1981), Essentials of Brownian motion and diffusion, Math. Surveys, no. 18, Amer. Math. Soc., Providence, R.I.

P. A. Loeb (1975), Conversion from nonstandard to standard measure spaces and applications in probability theory, Trans. Amer. Math. Soc. 211, 113-122.

(1979), An introduction to nonstandard analysis and hyperfinite probability theory, Probabilistic

Analysis and Related Topics, vol. 2 (A. Barucha-Reid, ed.), Academic Press, New York.

M. Reimers (1986), Univ. of British Columbia, Ph.D. dissertation.

S. Roelly-Coppoletta (1986), A criterion of convergence of measure-valued processes: application to measure branching processes, Stochastics 17, 43-65.

C. A. Rogers (1970), Hausdorff measures, Cambridge Univ. Press, Cambridge.

C. A. Rogers and S. J. Taylor (1961), Functions continuous and singular with respect to a Hausdorff measure, Mathematika 8, 1-31.

J. B. Walsh (1986), An introduction to stochastic partial differential equations, École d'Été de Probabilités de Saint Flour XIV-1984, Lecture Notes in Math., vol. 1180, Springer, New York, pp. 265-439.

S. Watanabe (1968), A limit theorem of branching processes and continuous state branching processes, J. Math. Kyoto. Univ. 8, 141-167.

U. Zähle (1984), The fractal carrying dimension of a critical multiplicative measure diffusion process, Technical Report N/84/79, Friedrich-Schiller-Universität Jena.

Department of Mathematics, University of British Columbia, Vancouver, British Columbia, V6T 1Y4 CANADA 